\title{
X-Ray Micro- and Nano-Diffraction Imaging on Human Mesenchymal Stem Cells and Differentiated Cells
}

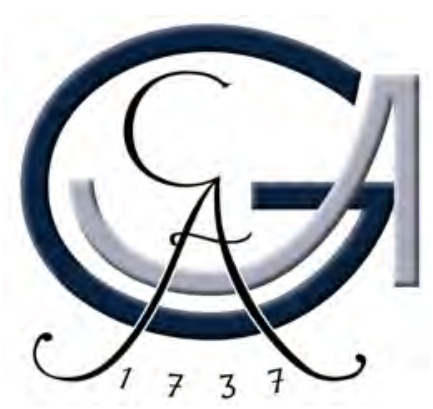

Dissertation

zur Erlangung des mathematisch-naturwissenschaftlichen

Doktorgrades

"Doctor rerum naturalium"

der Georg-August-Universität Göttingen

\author{
vorgelegt von \\ Marten Bernhardt \\ aus Henstedt-Ulzburg
}

Göttingen, 2016

Institut für Röntgenphysik 


\section{Mitglieder des Betreuungsausschusses:}

Referent: $\quad$ Prof. Dr. Tim Salditt

Institut für Röntgenphysik,

Georg-August-Universität Göttingen

1. Korreferent: Dr. Florian Rehfeldt

Drittes Physikalisches Institut - Biophysik,

Georg-August-Universität Göttingen

2. Korreferent: $\quad$ Prof. Dr. Andreas Janshoff

Institut für Physikalische Chemie,

Georg-August-Universität Göttingen

\section{Weitere Mitglieder der Prüfungskomission:}

Dr. Dieter Klopfenstein

Drittes Physikalisches Institut - Biophysik,

Georg-August-Universität Göttingen

Dr. Alexander Egner

Laser-Laboratorium Göttingen e. V.

Prof. Dr. Ulrich Parlitz

Max-Planck-Institut für Dynamik und Selbstorganisation

\section{Tag der mündlichen Prüfung:}




\section{Contents}

\begin{tabular}{lll}
\hline & Introduction & 1
\end{tabular}

2 SAXS of Biological Cells: Sample Preparation \& Instrumentation 7

$2.1 \quad$ X-Ray Instrumentation $\ldots \ldots \ldots \ldots \ldots$. . . . . . . . . . . . . . . . . .

2.2 Resolution \& Basic Scanning SAXS Mappings . . . . . . . . . . . . . . . 10

2.2.1 Real Space Resolution \& Basic Scanning SAXS Mappings . . . . 10

$2.2 .2 \quad$ Reciprocal Space Resolution $\ldots \ldots \ldots \ldots$

2.3 Sample Preparation $\ldots \ldots \ldots \ldots \ldots \ldots$

$2.3 .1 \quad$ Overview of Preparation Methods . . . . . . . . . . . . . . . . . . . . . . . . . . . . . 20

2.3 .2 Cell Culture $\ldots \ldots \ldots \ldots \ldots$

3 Cellular SAXS: Model independent Data Analysis \& Fitting 23

3.1 Principal Component Analysis (PCA) . . . . . . . . . . . . . . . . . . . . . 24

$3.1 .1 \quad$ Principal Proceeding . . . . . . . . . . . . . . . . . 25

3.1.2 First Applications on Model \& Real Data . . . . . . . . . . . . . . . . . . . . . . . . 37

3.1.3 Multiple Streak Analysis . . . . . . . . . . . . . . . . . 31

3.1.4 Comparison of Anisotropy Analysis Tools . . . . . . . . . . . . . 32

3.2 Comparative Analysis of Fluorescence Microscopy \& Scanning SAXS . . 36

3.3 Automated Analysis of Radial Intensity Profiles . . . . . . . . . . . . . . 41

\begin{tabular}{|lll}
4 & A SAXS Study of Stem Cells & 45
\end{tabular}

4.1 Micro-SAXS on Freeze-Dried Cells $\ldots \ldots \ldots$. . . . . . . . . . . . 46

4.2 Micro-SAXS Analysis: Statistics \& Definition of Cellular Diffraction Ob-

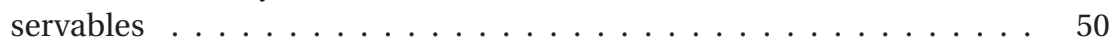

$4.3 \quad$ Increasing The Real-Space Resolution by Nano-SAXS . . . . . . . . . . . 53

4.4 Nano-SAXS from Cryoprotected Cells . . . . . . . . . . . . . . . . . . . . . . . . 54

4.5 Nano-SAXS from Frozen-Hydrated Cells . . . . . . . . . . . . . . . . . . . . . . . . . . . . . . . 59

4.6 Scanning SAXS from Hydrated Cells . . . . . . . . . . . . . . . . . 62

\begin{tabular}{|lll}
5 & A SAXS Study of Cardiomyocytes & 67
\end{tabular}

$5.1 \quad$ Micro-SAXS on Freeze-Dried Cells $\ldots \ldots \ldots$. . . . . . . . . . . . . . . . . . .

5.2 Increasing the Statistics by Large Area Scans . . . . . . . . . . . . . . . . . . . . . . . . . . . . . . . .

5.3 Nano-SAXS on Cardiac Tissue Cells . . . . . . . . . . . . . . . . . . . . . . . . . . . . .

5.4 Alive Recordings . . . . . . . . . . . . . . . . . . 86 


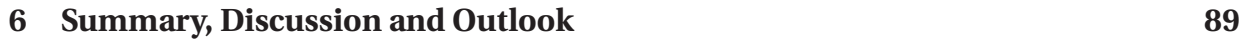

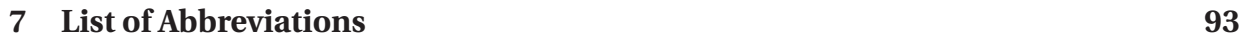

\begin{tabular}{lll}
\hline & Bibliography & 95
\end{tabular}

$\begin{array}{lll}9 & \text { Danksagung } & 103\end{array}$

10 Appendix 107

10.1 X-Ray Instrumentation (Detailed) $\ldots \ldots \ldots$. . . . . . . . . . . . . . . . . . . . . . . . . . . . . . 11

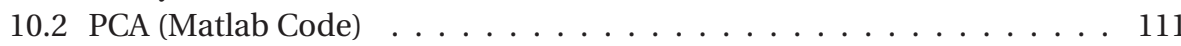

10.3 Stem Cells: Statistics \& Cellular Diffraction Observables. . . . . . . . . . 115

10.4 Grid Plunger Protocol . . . . . . . . . . . . . . . . . . . . . . . . . . . . . . . . . . . . . . . . 117

10.5 Mobile Incubator . . . . . . . . . . . . . . . . . . . . 122 


\section{Section 1}

\section{Introduction}

Biological cells are the fundamental units of life, enabling the existence of bacteria to sophisticated multi-cellular organisms. They share common features and functions, such as DNA encoding for reproduction, RNA transcription for protein synthesis, or the assembly of lipid membranes. At the same time, the diversity of purposes which particular cells have to fulfill requires an adaptation of cellular attributes. This concerns their morphology, structure, transcriptome and proteome and results in the wealth of different cell types known today. For the example of an adult human being, the estimated total number of cells is $10^{13}$ (1), grouped into a yet unknown number of different cell lines. At the same time, all these cells originate from only one single progenitor cell in the early stage of development. This so-called totipotent embryonic stem cell proliferates, starting a differentiation process. In this process, the entire spectrum of cells is successively generated. All subsequent stem cells can be classified by their differentiation capabilities and are denoted as pluri-, multi-, oligo- down to unipotent adult stem cells, finally leading to differentiated cell lines.

One example for pluripotent stem cells are human mesenchymal stem cells (hMSCs) of the bone marrow, capable of either self-renewing or differentiating. The cellular fate of hMSCs driven towards differentiation can be controlled by biochemical induction (2. 3) as well as the physical properties of their micro-environment (3). hMSCs provide a broad range of descendant cell lines such as osteocytes, myoblasts, chondrocytes, fibroblasts and adipocytes (4). Interestingly, recent studies indicate that hMSCs might provide a cellular source for cardiomyocytes as well [5- -8$]$.

Actin plays a critical part in this process. It is a widely distributed protein present in 
all eukaryotic cells in a steady state equilibrium between the (monomeric) globular (g-actin) and the filamentous (f-actin) state. Together with microtubules and intermediate filaments f-actin forms a protein network better known as the cytoskeleton of a cell. This network is essential for the cell's mechanical stability, provides a vital active transport system for chemical substances and, for special cell types, enables the cell to exert active forces. An overview of key aspects of the cytoskeleton is given by Alberts $e t$ al. (1).

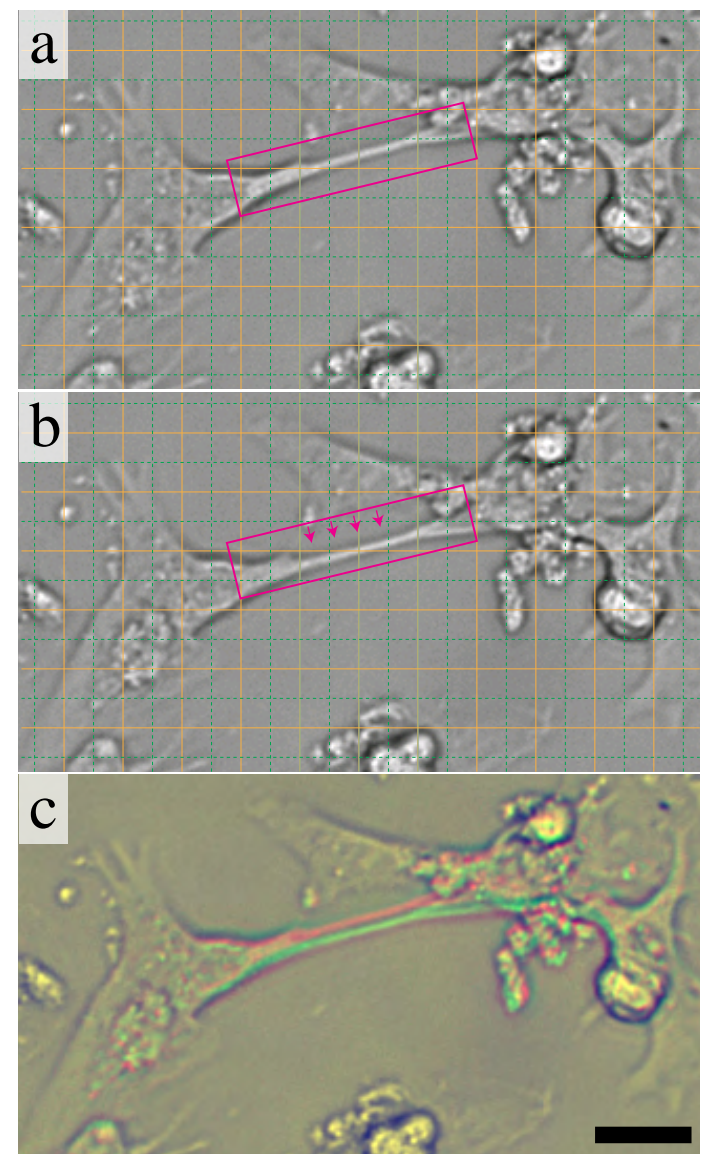

Figure 1.1: (a-c) Cardiomyocytes in a relaxed (see a) and in a tense state (see b) shifting local structures by a concerted contraction of myofibrils. A superimposition showing the relaxed state as the red and the tense state as the green RGB-channel, further illustrates these shifts (see c). Scale bar: $20 \mu \mathrm{m}$.
The structural attributes of different cell types are quite distinct (9) and are established successively throughout the differentiation process. In this process, actin together with its canonical counterpart myosin forms contractile fiber bundles, the so-called stressfibers. The formation of these bundles allows the cell to sense the mechanical properties of its immediate surrounding (mechanotransduction) via the anchor points (focal adhesions), which is decisive for the cell's fate. In fact, mechanosensing tends to be more selective during the differentiation process than any biochemical induction (3). The corresponding differentiated cell lines show a fiber distribution that can range from highly oriented to rather diffuse networks (10).

In particular (cardiac) muscle cells rely on a highly ordered system and establish their contractile forces by a locomotive apparatus based on myofibrils. Myofibrils consist of a strict alignment of alternating actin and myosin segments. One subunit called a sarcomere is repeatedly elongated throughout the entire cell. With many myofibrils formed in each cell and a constructive collab- 
oration of muscle cells inside the organ, this composition is the key to facilitating a macroscopic contraction of the entire heart muscle in one concerted movement.

Fig. 1.1 illustrates the contraction of cardiomyocytes showing a micrograph of cells in a relaxed state, see $(a)$, and in a tense state, see $(b)$. The respective translational shift of local areas become immediately apparent in a superimposition of images, see $(c)$, depicting the relaxed state in the red and the tense state in the green RGB-channe 1 Furthermore, Fig. $1.2(a-b)$ shows the fluorescence micrograph of a (striated) neonatal rat cardiomyocyte, see $(a)$, together with a non-striated cell, see $(b)$, also originating from cardiac tissue. The striation in the fluorescence signal of ( $a$ ) originates from the alternation of actin-dense and actin-poor regions within the myofibrils, which is absent in non-muscular cell lines. The periodicity of the striated pattern in the relaxed state is around $2.0 \mu \mathrm{m}(1)$.

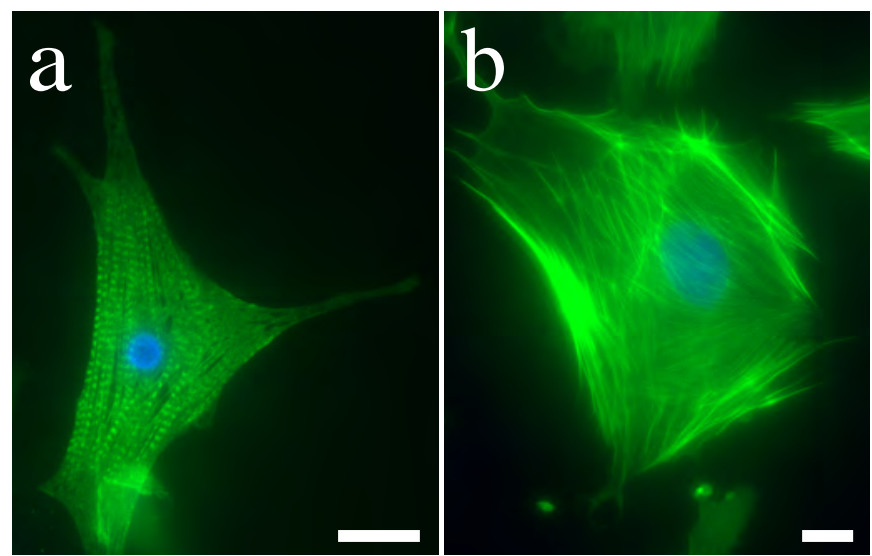

Figure 1.2: ( $(a-b)$ Visible light fluorescence micrograph of a striated cardiomyocyte, see (a), and a non-striated cardiac tissue cell, see (b). Stained actin is shown in green, the nucleus is shown in blue. Scale bars: $20 \mu \mathrm{m}$. Data recorded by M. Eckermann (11).

Both stress-fibers and myofibrils include many fascinating structural properties on the meso- and nanoscale (9] 12). Addressing such properties with adequate precision requires innovative high resolution imaging techniques. One of such techniques is x-ray diffraction (XRD) encoding structural details of sub-nanometer scales in the diffraction signal (12). However, state of the art X-ray focusing capabilities have restricted investigators to average over macroscopically large sample domains. Recent progress in $x$-ray optics based on a variety of optical elements such as Fresnel zone plates (13), compound refractive lenses (14-16) and elliptical Kirkpatrick-Baez (or KB) mirrors (17-20)

$\overline{1}$ The blue channel is set to an arbitrary value in order to avoid an intense yellow engraving. 
have made focal dimensions in the submicron-range possible. Further studies have shown, that a focal spot size of $5 \mathrm{~nm}$ is currently feasible (19, 21). While this is not practical for biological cells so far (due to a tiny working distance), recent x-ray instruments currently provide a spot size of typically around a few hundred nanometers for cellular recordings (20). This resolution is already sufficient to assign the scattering signal to subcellular compartments such as the different organelles of a cell. A motorized translation stage is used to successively scan the sample, so that for each probing spot one obtains a 2D diffraction pattern encoding the local electron density distribution, which finally leads to a complete mesh of 2D diffraction patterns of the scan area (22-24). We will denote this data recording scheme as scanning small angle $\mathrm{x}$-ray scattering or scanning SAXS.

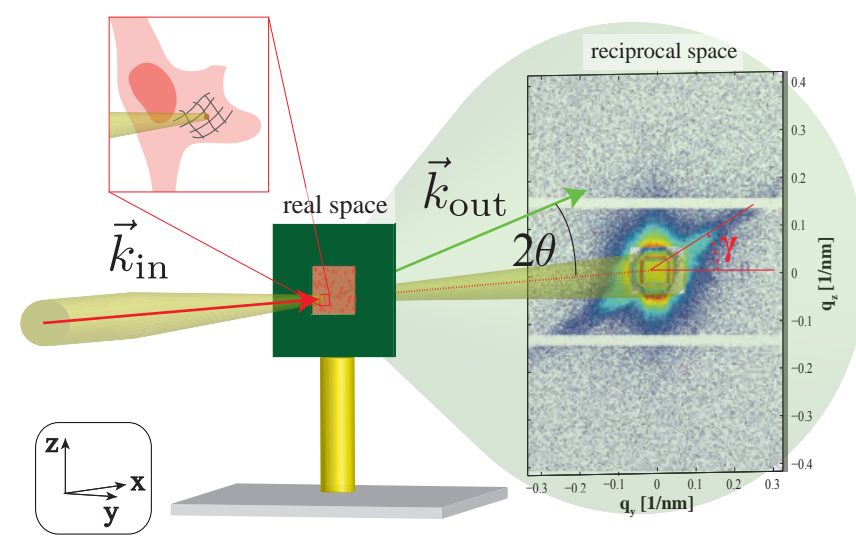

Figure 1.3: Basic concept of $x$-ray diffraction from a biological cell: The sample is successively translated through the focal plane of a focusing optics (not shown).

Fig. 1.3 illustrates the basic concept of x-ray diffraction: A high-flux x-ray beam generated from a synchrotron source $\left(I_{0} \approx 10^{11} \mathrm{ph} . / \mathrm{s}\right)$ is focused to probe the sample at its focal spot. Photons described by an incoming wave vector $\vec{k}_{\text {in }}$, with $\left|\vec{k}_{\text {in }}\right|=2 \pi / \lambda$, are scattered by the sample leading to an outgoing wave vector $\vec{k}_{\text {out }}$. They are registered by a flat panel single photon counting detector as a function of the components $q_{y}$ and $q_{z}$ of the scattering vector $\vec{q}$ with

$$
\vec{q}=\vec{k}_{\text {out }}-\vec{k}_{\text {in }}
$$

and

$$
\sin \left(\frac{2 \theta}{2}\right)=\frac{|\vec{q}| / 2}{2 \pi / \lambda} \Leftrightarrow|\vec{q}|=\frac{4 \pi}{\lambda} \sin \left(\frac{2 \theta}{2}\right)
$$


The structural attributes of the sample at the probing spot determine the photon distribution on the detector. The $q$-range is adapted before the experiment with respect to the structure size of interest, and primarily depends on the photon energy $E_{\mathrm{ph}}$. as well as the sample-to-detector distance. When performed at small opening angles $2 \theta$, nanosize substructures of cells can be investigated. In the example of Fig. 1.3 the detector image indicates the presence of an extended (i. e. filamentous) structure at the probing spot, which can be deduced from the anisotropic (and thus $\gamma$-angle dependent) nature of the diffraction pattern.

As will be described in the following sections in more detail, one can convert the collected diffraction data into a real space mapping of the sample, e. g. by integration of each diffraction signal. Such maps result in overviews of the entire scan area. The corresponding real space resolution is limited by the beam dimensions in focus. Furthermore, one can post-process scattering data in reciprocal space. The reciprocal space resolution is limited by the scattering strength of the signal. Well-written introductions to SAXS recordings are published by Schnablegger and Singh (25) as well as Als-Nielsen and McMorrow (26).

So far, a variety of recent studies have proven that scanning SAXS on biological matter and in particular single cells is feasible: Weinhausen et al. performed a first comparative study on human carcinoma cells including a successful correlation between the visible light fluorescence micrograph and the structure orientation of the local diffraction patterns (27). This project has also led to first scans on (initially) alive cells based on a home-built microfluidic chamber that is applicable for the hard x-ray regime (28, 29). Follow up experiments and theoretical work by Hémonnot et al. have enabled the further exploitation of data by tracing back the occurring modulations of single diffraction patterns to concrete (model-based) structures of the keratin network. A work of Wilke et al. illustrates the benefits of combining coherent x-ray imaging with nanodiffraction of a few nanometers resolution in reciprocal space (30). Priebe et al. have performed experiments on Dictyostelium discoideum aiming at the motile apparatus of Amoeba cells in the starvation state (31). Results reveal pronounced anisotropic scattering patterns on the perimeter of the cells. The corresponding structure orientations have been identified to be predominantly parallel to the cellular membrane and are attributed to the actomyosin cortex of the cell. Töpperwien et al. have complemented these results by a study on f-actin in vitro suspensions cross-linked with $\alpha$-actinin (32). Besides the biophysical content of all of these studies, they have also led to a whole pool of newly developed data analysis tools enabling the further interpretation of a dataset.

Moreover, these works have proven, that scanning SAXS is capable of complementing 
the repertoire of high resolution imaging techniques (33) by means of the basic principle it relies on, thus registering all cellular constituents according to local electron densities regardless of any fluorescent labeling. When enhanced by analysis algorithms, simulations as well as x-ray-based imaging (e.g. ptychography, holography and tomography (30, 34) ) or non-x-ray-based high resolution techniques (e. g. PALM and STEDnanoscopy (35-39)), the corresponding patterns can be further interpreted. Recordings are feasible on samples in various preparation states including freeze-drying, cryo or chemical fixation, or even an alive preparation.

The present work continues towards establishing scanning SAXS as a powerful tool by further development of analysis routines and the presentation of results on wild-type eukaryotic cell lines including stem cells and cardiomyocytes. In particular cardiomyocytes are suitable for cellular SAXS studies since they comprise myofibrils with multifaceted structural properties, that were previously addressed by x-ray diffraction experiments from muscle (12, 40, 41), but not on a sub-cellular level.

This work is organized as follows: After this brief introduction, fundamentals of scanning SAXS analysis, sample preparation protocols and setup parameters are detailed in Sec. 2 First results of this work are presented in Sec. 3 describing self-developed data analysis tools that enable the reduction of diffraction patterns to a few directly accessible observables. Implemented in an automated scheme, this allows the examination of an entire scan in view of a particular aspect. Sec. 4 illustrates the potential of the scanning SAXS method on a first example of stem cells and differentiated cell lines, showing that scanning SAXS can be evolved into a powerful tool to complement structural observations in stem cell research. Sec. 5 then addresses the structural attributes of neonatal cardiac tissue cells (NRCTCs) also demonstrating the capabilities of the data analysis tools developed in this work. The work closes with a summary, a discussion and an outlook for the project in Sec.6 


\section{Section 2}

\section{SAXS of Biological Cells: Sample Preparation \& Instrumentation}

This section includes excerpts of (42) and (43).

This section refers to the $\mathrm{x}$-ray instrumentation and sample preparation: Sec. 2.1 shows a detailed overview of the experimental setup and further explains the principle of a scanning SAXS recording. Sec. 2.2 starts with a brief tutorial on how to determine the real and reciprocal space resolution and introduces basic analysis tools used for scanning SAXS mappings of the probed area. Sec. 2.3 closes by describing sample preparation protocols.

\subsection{X-Ray Instrumentation}

The data shown in this work rely on two scanning SAXS instruments: All nanoSAXS data were performed using the nanofocus of the Göttingen Instrument for Nano-Imaging with X-Rays (GINIX) at P10 beamline (PETRAIII/DESY) (23). The corresponding experimental setup will be explained in detail in the present section. All micro-SAXS recordings were performed at the cSAXS beamline of the Swiss Light Source, the instrumentation is described in more detail in Appendix Sec. 10.1 
Fig. $2.1(a-b)$ shows a sketch and a photograph of the basic experimental components of the nano-SAXS setup: A monochromatized $\mathrm{x}$-ray beam is defined by multiple slits and

\begin{tabular}{|c|c|c|c|c|c|}
\hline \multirow[t]{2}{*}{ location } & \multicolumn{2}{|c|}{ beamdiam. } & \multirow{2}{*}{$\begin{array}{r}\mathbf{E} \\
{[\mathrm{keV}]}\end{array}$} & \multirow{2}{*}{$\begin{array}{r}\lambda \\
{[\mathbf{p m}]}\end{array}$} & \multirow{2}{*}{$\begin{array}{r}\mathbf{I}_{\max } \\
{[\mathbf{p h} . / \mathbf{s}]}\end{array}$} \\
\hline & horz. & vert. & & & \\
\hline P10 (1 ${ }^{\text {st }}$ run $)$ & $320 \mathrm{~nm}$ & $250 \mathrm{~nm}$ & 7.9 & 156.9 & $1.29 \cdot 10^{11}$ \\
\hline $\mathrm{P} 10$ (2 ${ }^{\text {nd }}$ run $)$ & $370 \mathrm{~nm}$ & $180 \mathrm{~nm}$ & 13.8 & 89.8 & $1.38 \cdot 10^{11}$ \\
\hline $\mathrm{P} 10$ ( $3^{\text {rd }}$ run $)$ & $360 \mathrm{~nm}$ & $410 \mathrm{~nm}$ & 8.0 & 154.9 & $2.40 \cdot 10^{11}$ \\
\hline cSAXS & $54 \mu \mathrm{m}$ & $33 \mu \mathrm{m}$ & 8.7 & 142.5 & $1.44 \cdot 10^{11}$ \\
\hline
\end{tabular}
focused by a set of Kirkpatrick-Baez (KB) mirrors down to a spot size of typically about 200-400 nm full width half maximum (FWHM). The beam exits the vacuum at (1) probing the sample (4) in its focal spot $\approx 20 \mathrm{~cm}$ further downstream from the mirrors. (3) Two soft-edge apertures (44) trim the beam by blocking parasitic primary beam intensities.
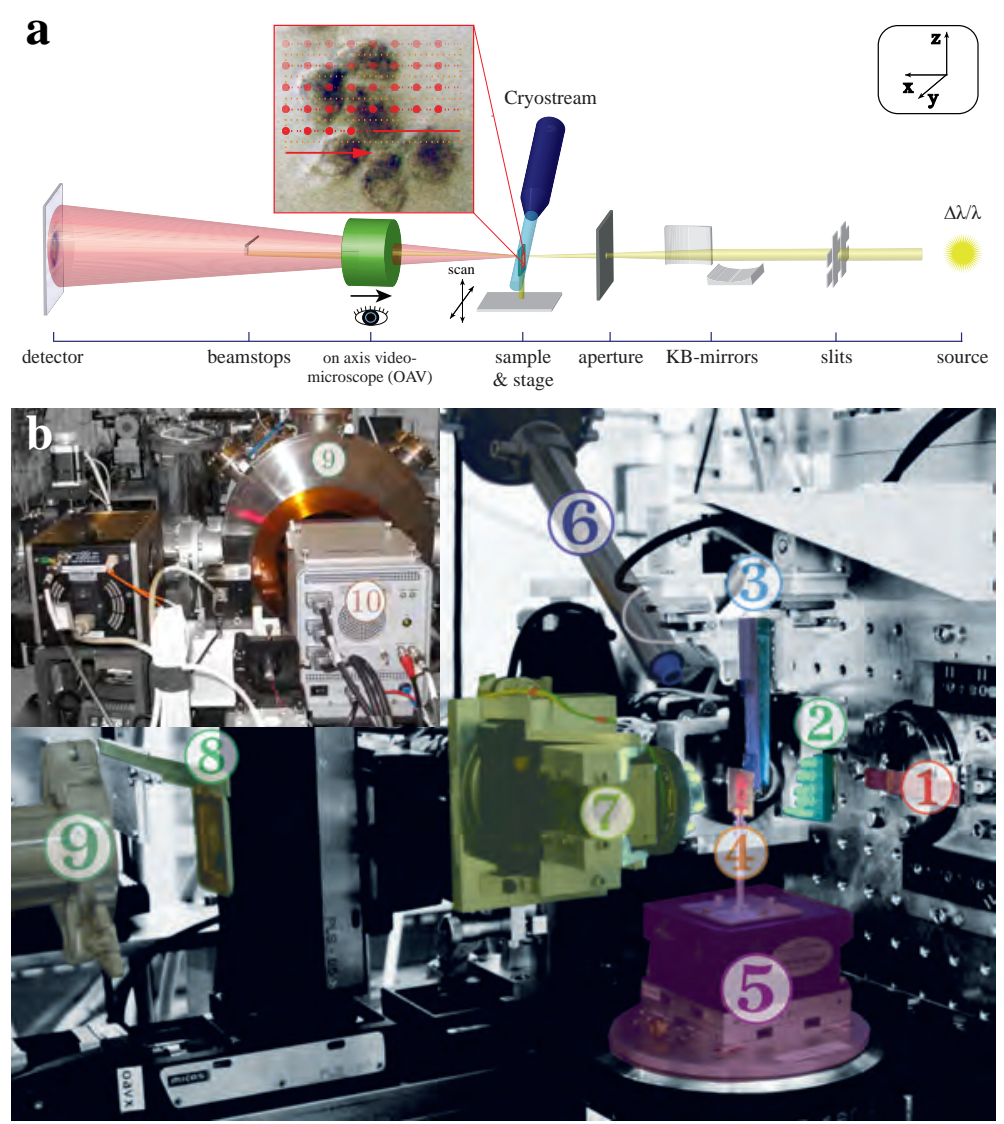

Figure 2.1: (a-b) Sketch and photograph of a nano-SAXS setup based on the Göttingen Instrument for NanoImaging with X-Rays (GINIX) at P10 beamline (PETRAIII/DESY) [20 23). Figure and caption adapted from 42). 
(5) A motorized stage enables sample translation with respect to the beam. (7) An on axis video-microscope $(\mathrm{OAV})$ with a reflection and a transmission illumination mode (see (7) \& (2)) provides a videoimage of the current region in focus, making possible the navigation on the sample in order to find a suitable scan region. The primary beam is blocked by (optionally semi-transparent) beam stops that can be introduced at different positions in front of or inside an evacuated tube, see (8) \& (9), which spans the sample-detector-distance. Scattered photons are recorded $\approx 5.1 \mathrm{~m}$ further downstream from the sample position by a single photon counting detector (Dectris, Switzerland), see (10. Optionally, cryogenic sample conditions can be applied using a cryostream system (6) (Oxford Instruments, UK).

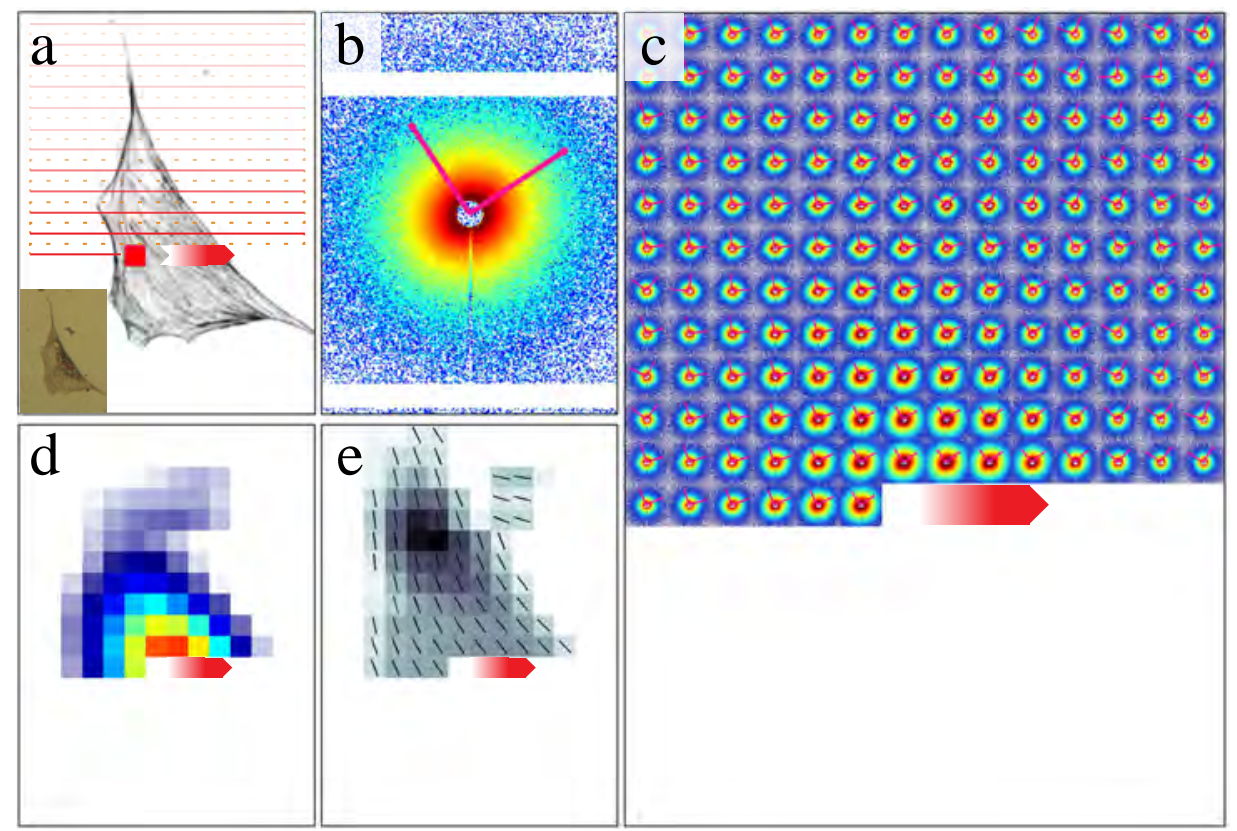

Figure 2.2: (a) Inverted visible light fluorescence micrograph together with the OAV-image (inset) of a freezedried NRCTC. (b) Single diffraction image of the approximate position marked in (a, red square). Pink arrows denote for the rescaled principal axes as will be introduced in Sec. 3.1.1 (c) Composite image of the running scan showing the center regions of each $2 D$-diffraction signal recorded so far. (d-e) X-ray dark field and PCA maps as will be further detailed in Sec.2.2.1 and Sec. 3.1.1

Fig.2.2 exemplarily shows the main results of a SAXS scan performed on a freeze-dried neonatal rat cardiac tissue cell (NRCTC). Fig. 2.2 (a) shows the (inverted) visible light fluorescence together with the OAV-micrograph (inset) of a particular scan area. Raster scan modus (sketched by red lines) leads to one diffraction pattern at each probing spot, see $(b-c)$. The depiction of Fig. 2.2 (c) will in the following be denoted as the 
composite image of a scan. (d) Online analysis such as an x-ray dark field evaluation (described in Sec. 2.2.1 provides the means to identify cell-related scattering signals in a straightforward manner. (e) Further analysis tools such as a principal component analysis (PCA) (described in Sec. 3.1.1 facilitate the mapping and quantification of local structural properties of the cells. Experimental parameters for micro- and nano-SAXS scans presented here are listed in Tab.2.1.

\subsection{Resolution \& Basic Scanning SAXS Mappings}

Scanning SAXS recordings comprise two different resolution concepts characterizing the experiment: The real space resolution of a scan as discussed in Sec.2.2.1 is derived from the step size $\Delta$ of a scan or the beam size at the probing spot. A high real space resolution allows the attribution of diffraction patterns to restricted regions of the sample enabling the assignment of diffraction features to sub-cellular local areas. The reciprocal space resolution as discussed in Sec.2.2.2 determines the resolution of structures after azimuthal averaging over a mean (background-corrected) cellular diffraction signal.

\subsubsection{Real Space Resolution \& Basic Scanning SAXS Mappings}

\section{Real Space Resolution}

The real space resolution of a scan equals to the scanning step size and is limited by the beam diameter at the sample position. Fig. $2.3(a-b)$ shows the nano-SAXS beam profiles in horizontal and vertical direction. Profiles were measured by scanning of a waveguide (here serving as an aperture) through the beam; each data point represents the total intensity of photons measured by the detector at the particular waveguide position. (a) Horizontal and (b) vertical profiles can be described by a Gaussian function. This provides the means to determine the corresponding FWHM-values for the curve, which we define as the beam dimensions.

For nano-SAXS scans, step sizes above the beam dimensions are chosen, ensuring that cellular scattering patterns are dominated by signals from intact rather than from beam-damaged cellular structures. Micro-SAXS recordings however allow steps down to a fraction of the beam size (in this work typically about a fifth to a tenth), since a comparable flux of about $10^{11} \mathrm{ph} . / \mathrm{s}$ is distributed over a much larger area. This becomes ap- 
parent when calculating the applied radiation dose following the scheme introduced in Sec. 4.4
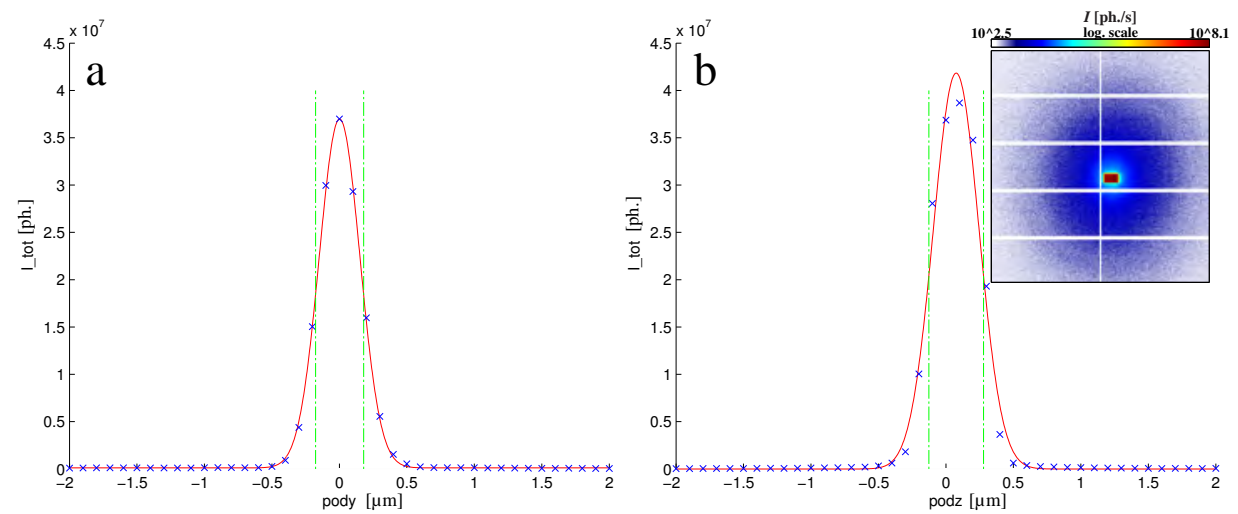

Figure 2.3: (a) Horizontal and (b) vertical nano-SAXS empty beam dimensions as measured by translation of a waveguide. (b, inset) shows the 2D far-field pattern of the empty beam. Figure and caption adapted from [43).
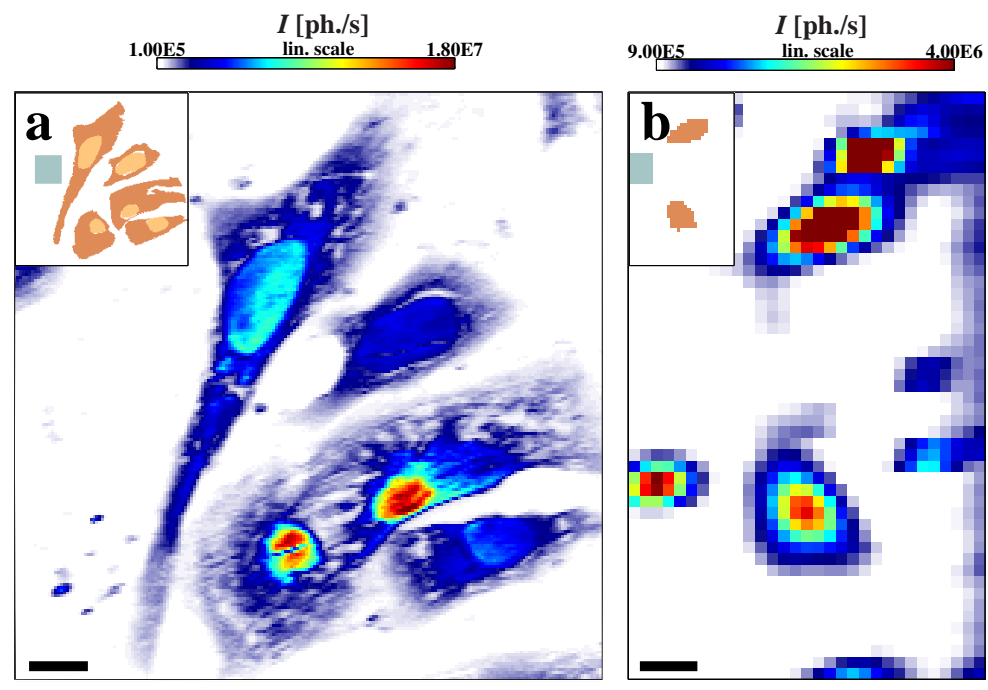

Figure 2.4: (a-b) Nano- and micro-SAXS maps of single cells together with the region-of-interest-masks (ROImasks, see insets) enabling the separation of nucleic, cytoplasmic and background regions in nanoSAXS datasets as well as cellular and background regions in micro-SAXS datasets. Scale bars: $10 \mu \mathrm{m}$ and $50 \mu \mathrm{m}$. Figure and caption adapted from [43].

A high real space resolution enables the identification of different cellular compartments such as the nucleus, the cytoplasm and the cellular cortex, see Fig.2.4 (a). A low 
real space resolution is well-suited for rapid scanning of large sample areas but at the same time prevents a further separation of cellular signals, see Fig. 2.4 (b).

\section{Scanning SAXS Mappings}

The scanning SAXS maps shown in Fig.2.4 result from the two-dimensional integration excluding primary beam (pb) and parasitic scattering (ps, for example caused by KBtails or scattering from apertures) by an adequate mask applied on each diffraction pattern,

$$
I_{\mathrm{df}}(a, b)=\int_{\mathbb{R}^{2} \backslash\{\{\mathrm{pb}\} \cup\{\mathrm{ps}\}]} I\left(y, z, q_{y}, q_{z}\right) \mathrm{d} q_{y} \mathrm{~d} q_{z} .
$$

Mappings of $I_{\mathrm{df}}(a, b)$ with respect to the relative scan position $(a, b) \in \mathbb{N}^{2}$ will in the following be denoted as the x-ray dark field of a scan.

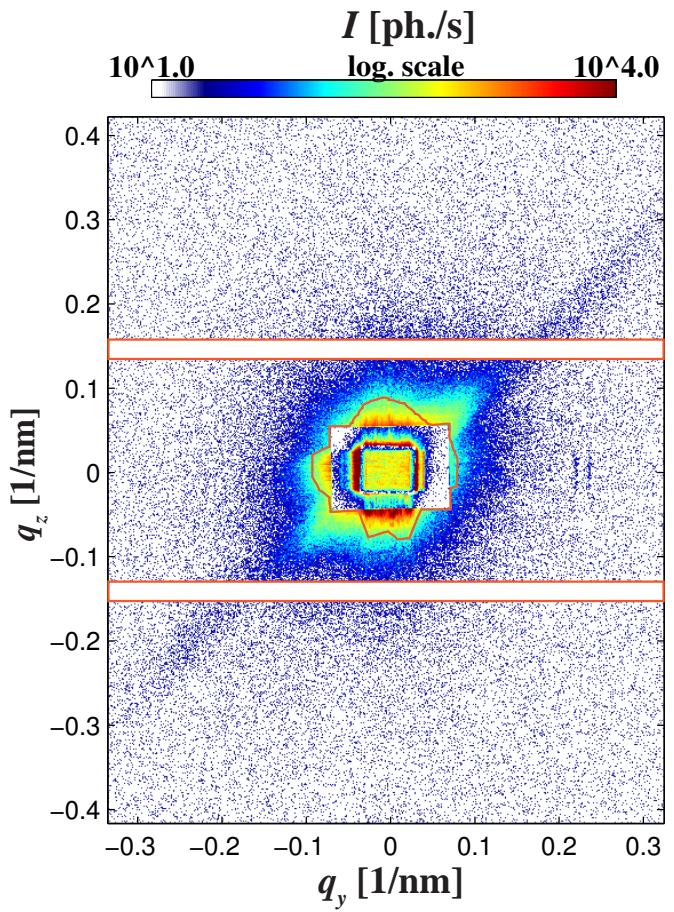

Figure 2.5: Single cellular 2D diffraction pattern. A detector mask (orange lines) enables $x$-ray dark field evaluation of the scan. Figure and caption adapted from [42).

Fig. 2.5 exemplarily shows a single cellular diffraction pattern including a pronounced anisotropic scattering portion. Orange lines mark the detector mask applied for x-ray 
dark field evaluation. The use of semi-transparent beam stops (indicated by rectangular shadows in the center) also enable other scanning SAXS contrast modes such as the $\mathrm{X}$-ray transmission resulting from an overall integral of all recorded photons

$$
I_{\mathrm{tr}}(a, b)=\int_{\mathbb{R}^{2}} I\left(y, z, q_{y}, q_{z}\right) \mathrm{d} q_{y} \mathrm{~d} q_{z}
$$

Furthermore, a differential phase contrast image (as implemented by my former colleague Dr. Robin Wilke) can be computed, quantifying the respective shift of the beam (center of mass) in either direction on the detector plane

$$
\begin{aligned}
d p c_{y, z}(a, b) & =\frac{1}{I_{\operatorname{tr}}(a, b)} \cdot \sum_{y, z} I_{\operatorname{tr}}(y, z) \cdot r_{y, z} \\
D P C_{y, z}(a, b) & =d p c_{y, z}(a, b)-\frac{1}{N_{a} \cdot N_{b}} \sum_{a, b} d p c_{y, z}(a, b) .
\end{aligned}
$$

$N_{a}$ and $N_{b}$ denotes for the dimensions of the scan (in pixel), $r_{y, z}$ is the current pixel position along the $y$ - or $z$-direction on the detector.

Fig. 2.6 shows the scanning SAXS evaluation of freeze-dried murine fibroblasts in all four modes with the dark field $I_{\mathrm{df}}$ in $(a)$, the transmission $I_{\mathrm{tr}}$ in $(b)$, and the differential phase shift $D P C_{y}$ and $D P C_{z}$ in $(c)$ and $(d)$. 

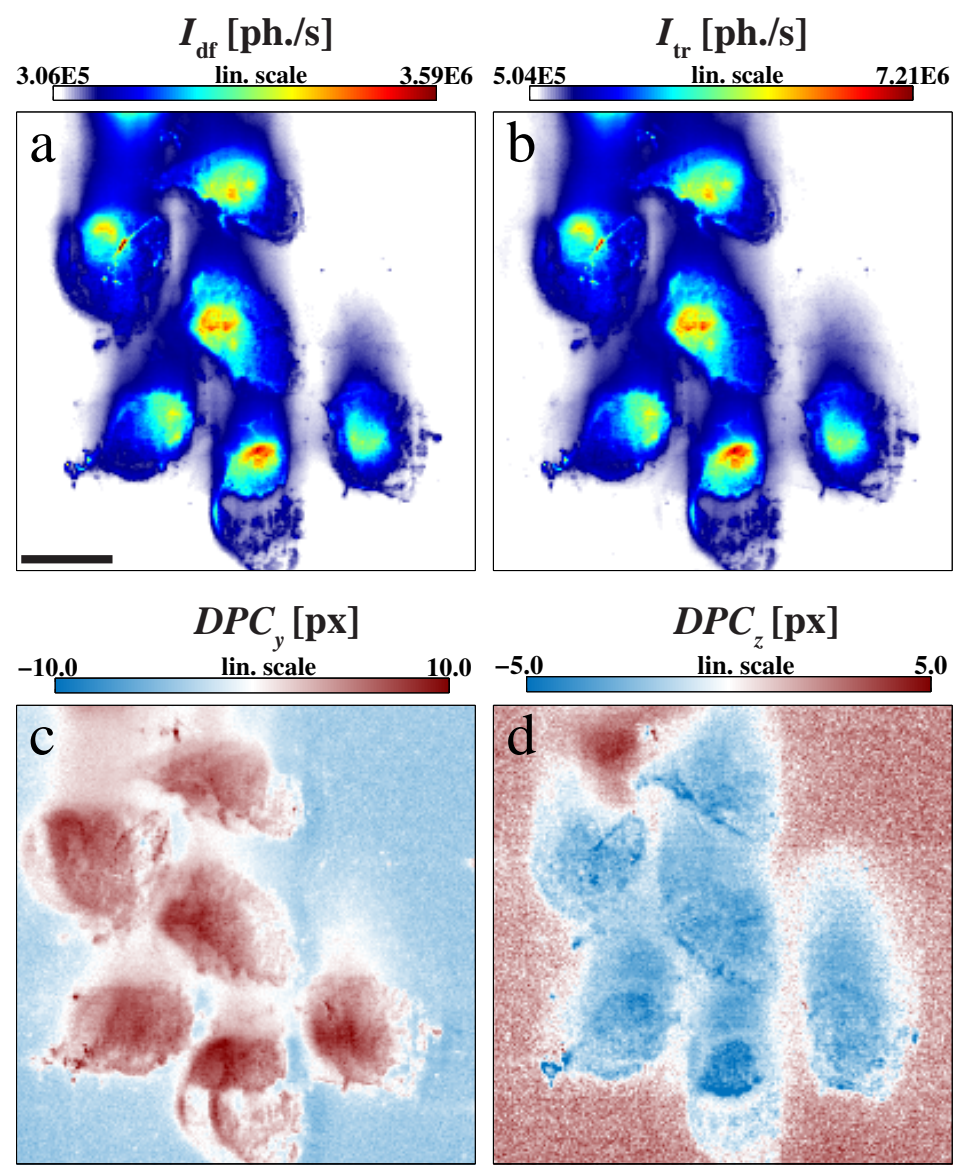

Figure 2.6: Different contrast modes for a nano-SAXS scan of freeze-dried murine fibroblasts on glass: (a) X-ray dark field intensities, (b) $x$-ray transmission intensities and (c-d) $x$-ray differential phase contrast in y- \& z-direction. Scale bar: $20 \mu \mathrm{m}$. Figure and caption adapted from [42). 


\subsubsection{Reciprocal Space Resolution}

The reciprocal space resolution $d_{\text {min }}$ depends on the setup (photon energy, $q$-range of the detector) as well as the scattering attributes of the sample. $d_{\min }$ is determined on radial intensity profiles $I\left(q_{\mathrm{r}}\right)$, with $q_{\mathrm{r}}=\sqrt{q_{y}^{2}+q_{z}^{2}}$, considering the cross-over point between the decay of the cellular signal and the background noise [30). Fig 2.7 ( $a$ c) shows the averaged 2D diffraction patterns of a (background-corrected) nucleic and cytoplasmic signal as obtained on a nano-SAXS, as well as the (backgroundcorrected) cellular signal as obtained on a micro-SAXS scan. Curves are computed based on data and ROI-masks shown in Fig. 2.4. The respective profiles $I\left(q_{\mathrm{r}}\right)$ result from a full azimuthal integration of the averaged 2D diffraction patterns, see $(d)$. To determine the minimum distance that can be resolved, radial decays have to be determined using adequate fitting approaches that allow a mathematical description of $I\left(q_{\mathrm{r}}\right)$ which enables to compute the respective cross-over points:

All nano-SAXS results presented here

\begin{tabular}{lccc}
\hline & spot & $\begin{array}{c}q_{\mathrm{r}} \\
\text { in }\left[\mathrm{nm}^{-1}\right]\end{array}$ & $\begin{array}{c}d \\
\text { in [nm] }\end{array}$ \\
\hline nano-SAXS & $(1)$ & 0.50 & 6.30 \\
& $(2)$ & 0.49 & 6.36 \\
micro-SAXS & $(3)$ & 0.44 & 7.09 \\
& (4) & 0.02 & 155.58 \\
\hline
\end{tabular}

Table 2.2: Results for $q_{1-4}$ and $d_{1-4}$ for cross-over points (1)-(4) as shown in Fig. 2.4 can be described sufficiently well by a simple power law decay

$$
I_{\text {nano }}\left(q_{\mathrm{r}}\right)=a \cdot q_{\mathrm{r}}^{b}+c
$$

However, micro-SAXS diffraction occasionally reveal several decays denoting for lowand high- $q_{\mathrm{r}}$-regions, that require a more elaborate fitting function (11) such as

$$
I_{\text {micro }}\left(q_{\mathrm{r}}\right)=\frac{1}{\left(a_{1} \cdot q_{\mathrm{r}}^{\beta}+a_{2} \cdot q_{\mathrm{r}}^{2 \beta}\right)^{2 / \beta}}+b,
$$

The results (see Tab. 2.3 \& Tab. 2.4 lead to the graphs depicted as thick pink lines. Thin pink lines mark the overall decays of a respective region, as derived from $\Delta I\left(q_{\mathrm{r}}\right)=a \cdot q_{\mathrm{r}}^{b}$ for nano-SAXS, and $\Delta I_{\text {low }}\left(q_{\mathrm{r}}\right)=\frac{1}{a_{1}^{2 / \beta} \cdot q_{\mathrm{r}}^{2}}$ as well as $\Delta I_{\text {high }}\left(q_{\mathrm{r}}\right)=\frac{1}{a_{2}^{2 / \beta} \cdot q_{\mathrm{r}}^{4}}$ for low- and high$q_{\mathrm{r}}$-values of micro-SAXS results.

The background signal is determined from the mean value of all (smoothened) data points within a predefined region right after the cellular signal pass into the background noise. The corresponding cross-over points (1)-(3) at value $q_{1-3}$ determine the reciprocal space resolution of the signal.

Let $\varepsilon_{\mathrm{nn}}, \varepsilon_{\mathrm{nc}}$ and $\varepsilon_{\mathrm{mc}}$ be the offsets for the nano-SAXS nucleic (nn), the nano-SAXS 
cytoplasmic (nc) and the micro-SAXS cellular (mc) regions, it follows

$$
\varepsilon_{\mathrm{nn}, \mathrm{nc}}=a_{\mathrm{nn}, \mathrm{nc}} \cdot q_{1,2}^{b_{\mathrm{nn}, \mathrm{nc}}}+c \Leftrightarrow q_{1,2}=\left(\frac{\varepsilon_{\mathrm{nn}, \mathrm{nc}}-c_{\mathrm{nn}, \mathrm{nc}}}{a_{\mathrm{nn}, \mathrm{nc}}}\right)^{1 / b_{\mathrm{nn}, \mathrm{nc}}}
$$

for cross-over points (1) \& (2),

$$
\varepsilon_{\mathrm{mc}}=\frac{1}{a_{2}^{2 / \beta} \cdot q_{3}^{4}} \Leftrightarrow q_{3}=\left(\frac{1}{a_{2}^{2 / \beta} \cdot \varepsilon_{\mathrm{mc}}}\right)^{1 / 4}
$$

for cross-over point (3) and

$$
\frac{1}{a_{1}^{2 / \beta} \cdot q_{4}^{2}}=\frac{1}{a_{2}^{2 / \beta} \cdot q_{4}^{4}} \Leftrightarrow q_{4}=\left(\frac{a_{1}}{a_{2}}\right)^{1 / \beta}
$$

for cross-over point (4). The calculated minimum distance that can be resolved is determined by

$$
d_{1-3}=\pi / q_{1-3}
$$

The corresponding results around $7 \mathrm{~nm}$ are in good agreement with other SAXS studies using an equivalent setup (31).

\begin{tabular}{lccc}
\hline & $\left.\left.\begin{array}{c}a \\
{\left[\left(\frac{\mathrm{nm} \cdot \mathrm{ph} .}{\mathrm{s}}\right)\right.}\end{array}\right)^{b}\right]$ & $b$ & $c$ \\
& {$[1]$} & {$[\mathrm{ph} . / \mathrm{s}]$} \\
\hline nuc.-bckg. & $2.8 \cdot 10^{-2}$ & -3.98 & $8.4 \cdot 10^{-2}$ \\
cytop.-bckg. & $6.6 \cdot 10^{-3}$ & -4.17 & $3.9 \cdot 10^{-2}$ \\
\hline
\end{tabular}

Table 2.3: Fit results based on Eq. 2.5 for a nano-SAXS dataset.

\begin{tabular}{ccccc}
\hline & $\begin{array}{c}a_{1} \\
{\left[\left(\frac{\mathrm{s} \cdot \mathrm{nm}}{\mathrm{ph}}\right)^{\frac{\beta}{2}}\right]}\end{array}$ & $\begin{array}{c}a_{2} \\
{\left[\left(\frac{\mathrm{s} \cdot \mathrm{nm}}{\mathrm{ph} .}\right)^{\frac{\beta}{2}}\right]}\end{array}$ & $\begin{array}{c}\beta \\
{[1]}\end{array}$ & $\begin{array}{c}b \\
{[\mathrm{ph} . / \mathrm{s}]}\end{array}$ \\
\hline cell-bckg. & 0.46 & $1.9 \cdot 10^{4}$ & 2.73 & $4.0 \cdot 10^{-2}$ \\
\hline
\end{tabular}

Table 2.4: Fit results based on Eq. 2.6 for a micro-SAXS dataset. 

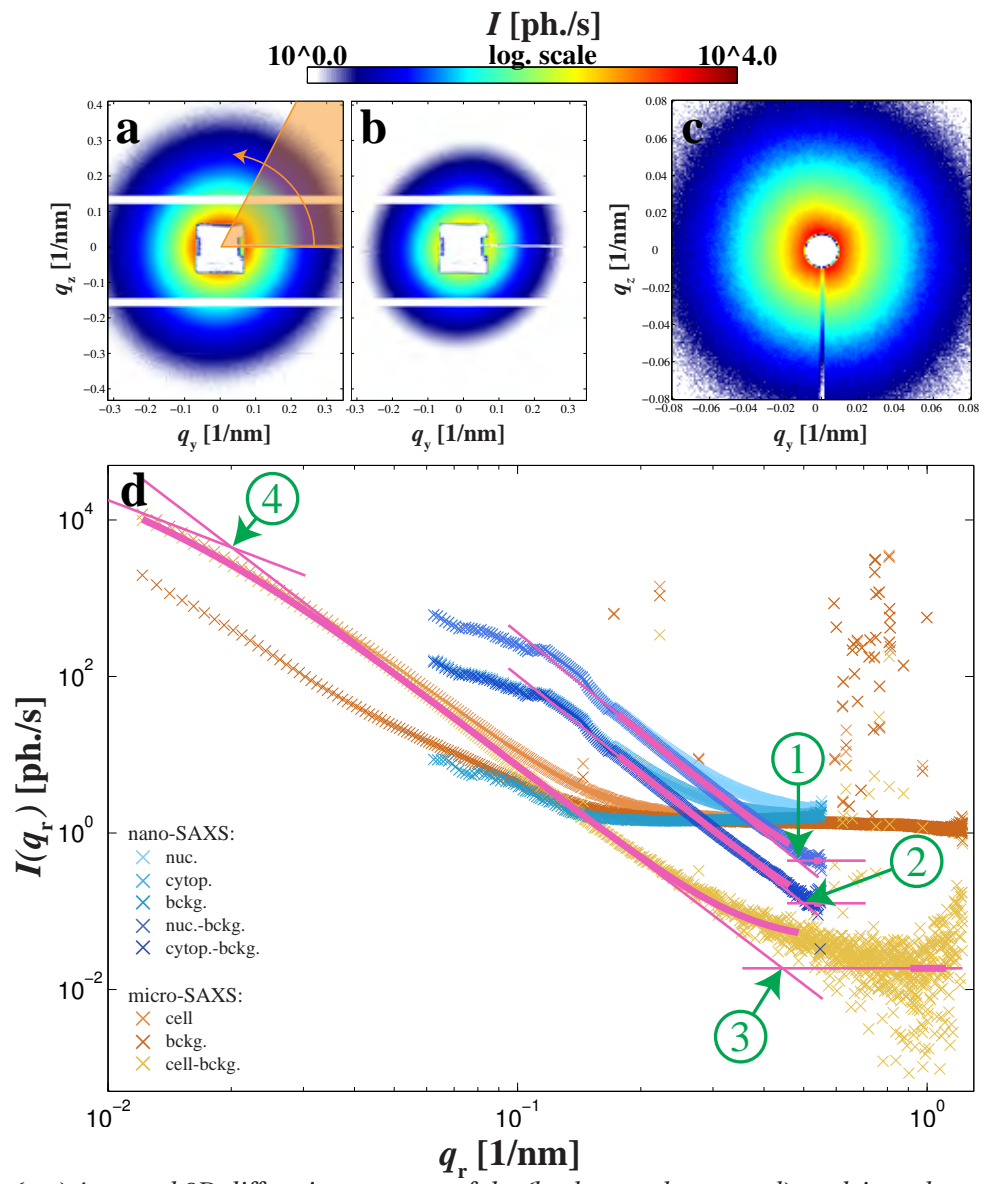

Figure 2.7: (a-c) Averaged 2D diffraction patterns of the (background-corrected) nucleic and cytoplasmic signal as obtained by nano-SAXS-, as well as the center region of the (background-corrected) cellular signal as obtained by micro-SAXS recordings. Results are based on the data and ROI-masks shown in Fig.2.4 (d) Radial intensity profiles $I\left(q_{\mathrm{r}}\right)$ resulting from averaged $2 D$ diffraction patterns of the different areas defined in Fig.2.4 Bold pink lines denote the evaluation area for fitting the cellular or the background signal, respectively. Cross points (1)-(4) mark the positions defining the reciprocal space resolution, see (1)-(3), or the cross-over from one to the next power-law regime, see (4). Figure and caption adapted from [4] 


\subsection{Sample Preparation}

\subsubsection{Overview of Preparation Methods}

The versatility of many different synchrotron radiation beam lines enable the recording of samples in different preparation states, covering freeze-dried, frozen-hydrated, chemically fixated or even alive cells $(20,27,32)$. Fig. 2.8 summarizes the basic preparation steps for adherent (eukaryotic) cells.

Biochemical preparation: Cells, either obtained from previous cell cultivation or isolated from disassembled tissue, were suspended in nutrition medium and then settled on x-ray compatible substrates. After an incubation time of one up to a few days, samples were chemically fixated (also allowing fluorescent labeling of the cells) or left in the alive state for subsequent processing. Procedures are further detailed in Sec. 2.3.2

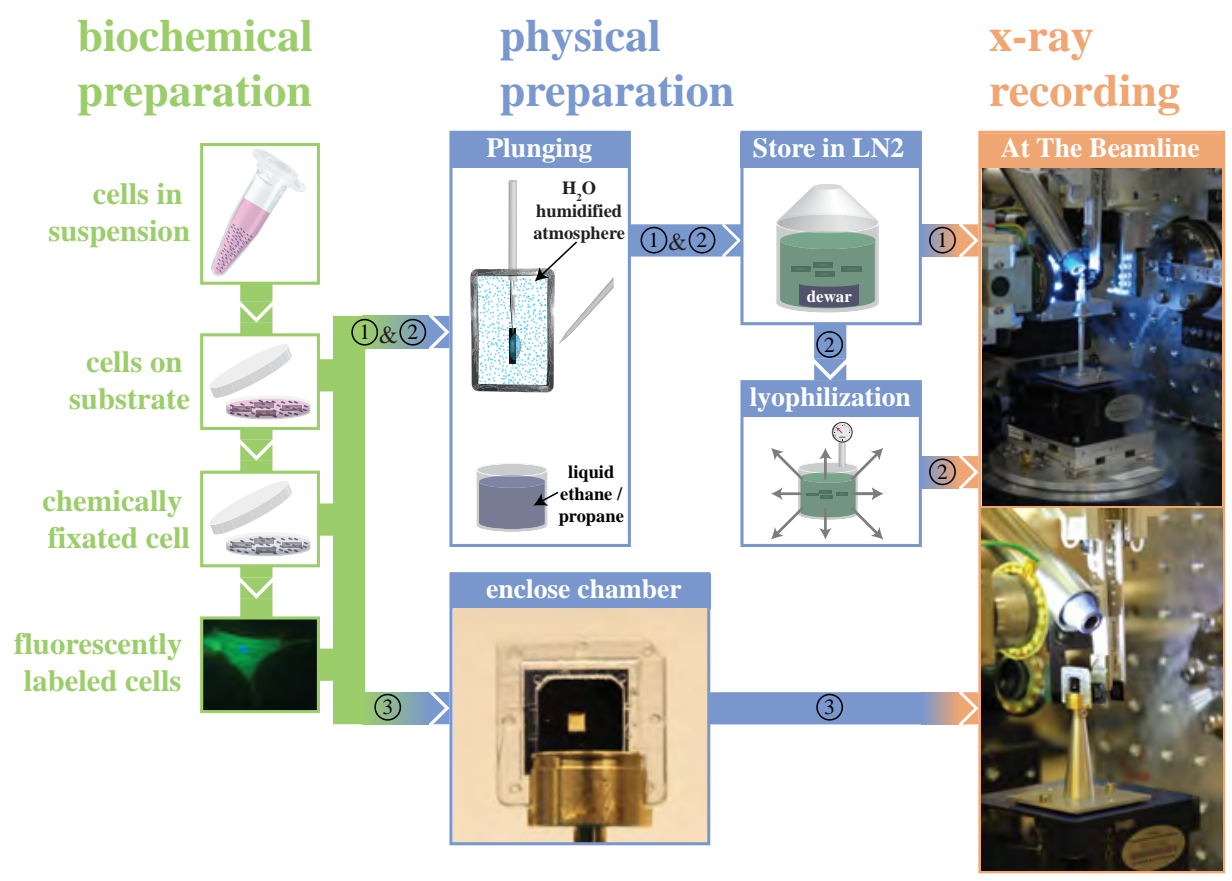

Figure 2.8: Overview of the basic sample preparation steps.

Physical preparation: Frozen-hydrated \& freeze-dried samples: (1)-(2) Cryopreparation of an (alive or chemically fixated) sample was performed using a commercially available grid plunging system (Leica EM GP, Leica Microsystems). While still covered in 
buffer solution, the sample was mounted in a water-saturated chamber using microtweezers (see Fig. 2.9 step 1). After several washing steps with a volatile buffer solution of $85.6 \mathrm{mM}$ Triethylammonium acetate in $\mathrm{H}_{2} \mathrm{O}$ (90358, Sigma-Aldrich), main parts of the remaining liquid were removed by manual blotting until the sample is covered by an ultrathin buffer layer (see Fig. 2.9, step 2-3).

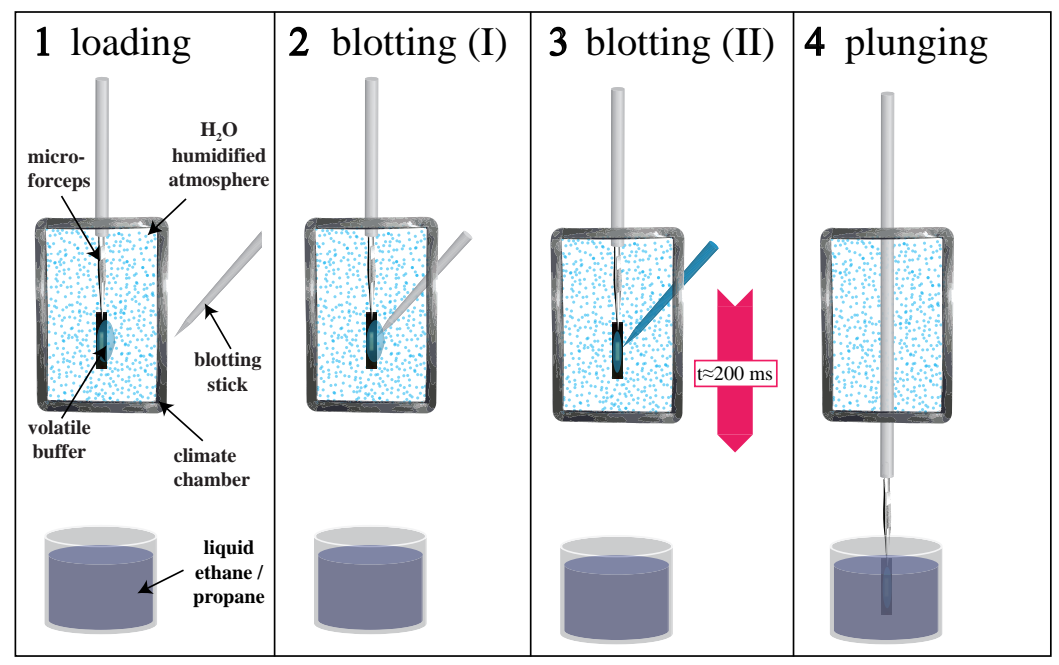

Figure 2.9: Main steps of the plunging procedure.

The sample was then plunged rapidly (in the order of $200 \mathrm{~ms}^{1}$ into an cryogenic bath of liquid ethane (purity: N35, Air liquide) or ethane \& propane mixture (37/63 vol.-\%, both purities: N35, Air liquide) with temperatures of around $-185^{\circ}$ or $-195^{\circ}$, respectively, see Fig. 2.9 step 4. A high cooling rate ensures the vitrifying effect of $\mathrm{H}_{2} \mathrm{O}$ in the buffer solution, i.e. the formation of an amorphous water layer, preserving cellular structures. Samples were then transferred into liquid nitrogen (LN2) for storage and could either be mounted directly on the sample stage in a frozen-hydrated state, see Fig. 2.8 (1), or could be first freeze-dried in a home-built vacuum chamber facilitating the sublimation of all volatile constituents, see Fig. 2.8 (2). While for frozen-hydrated samples a steady cryoprotection is mandatory, freeze-dried samples can be recorded with or without cryoprotection. For further handling instructions see also Appendix Sec. 10.4. Phase contrast- and fluorescence micrographs were taken at different preparation steps using a Zeiss Observer Z1 Microscope (Zeiss, Germany). Hydrated samples: For hydrated samples in the chemically fixated or alive state, cells were

1 according to a Leica-technician 
cultivated either in already established (commercially available) chambers with a sufficient gas exchange for buffer recovery or in open chamber architectures that were enclosed shortly before recording, see (3).

\subsubsection{Cell Culture}

\section{Stem Cells, Murine Myo- and Fibroblasts}

Large parts are taken from (42).

Cells: The cells used were obtained from the following sources: $N I H$-3T3s (murine embryonic fibroblasts): Deutsche Sammlung von Mikroorganismen und Zellkulturen (DSMZ), no. ACC 59; hMSCs (human mesenchymal stem cells): Lonza, no. PT-2501; C2C12 (murine myoblasts): DSMZ, no. ACC 565.

Cells were incubated at $37^{\circ} \mathrm{C}$ at $5 \% \mathrm{CO}_{2}$ in vent-cap culture flasks and on the day of splitting were washed with Dulbecco's phosphate-buffered saline (DPBS, D8537, Sigma-Aldrich) before trypsinization (0.05\%Trypsin-EDTA (25300-054, Gibco), esp. for hMSCs, or 1× Trypsin (59417C, Sigma-Aldrich)). Splitting was done every fourth day at most. Muscle-induced hMSCs (mi-hMSCs) were derived from naive hMSCs and remained attached up to 10 days. The medium was changed to muscle induction medium (MIM) as reported by Engler et al. [3] supporting material), more than a week before cryofixation.

All cell lines were cultivated in the third institute of physics - biophysics, whose staff, in particular the group of Dr. Florian Rehfeldt, work with these cells on a regular basis and can provide for the most up-to-date information in handling.

\begin{tabular}{ll}
\hline \multicolumn{1}{c}{ hMSC } & \multicolumn{1}{c}{ mi-hMSC (i.e. MIM) } \\
\hline DMEM (31885-023, Gibco) & DMEM (31885-023, Gibco) \\
$10 \%$ FBS (F2442, Sigma-Aldrich) & $20 \%$ FBS (F2442, Sigma-Aldrich) \\
$1 \%$ Pen/Strep & $1 \%$ Pen/Strep \\
& $50 \mu$ Hydroc.(H0888, later H0396, Sigma-Aldrich) \\
& $100 \mathrm{nM} \mathrm{Dexam.} \mathrm{(D4902,} \mathrm{Sigma-Aldrich)}$ \\
\hline \multicolumn{1}{c}{ C2C12 } & \multicolumn{1}{c}{ NIH-3T3 } \\
\hline DMEM (D6429, Sigma-Aldrich) & DMEM (D5796, Sigma-Aldrich) \\
$10 \%$ FBS (F0804, Sigma-Aldrich) & $10 \%$ FBS (F0804, Sigma-Aldrich) \\
$1 \%$ Pen/Strep & $1 \%$ Pen/Strep \\
\hline Table 2.5: Tabulated cultivation & media. Pen/Strep: 15140-122, Gibco or DE17- \\
602E, Lonza. &
\end{tabular}

Substrates: hMSC, mi-hMSC, NIH-3T3 \& C2C12 were prepared on silicon nitride ultrathin membranes (Silson, UK) of $1 \mu \mathrm{m}$-thickness or (borosilicate) glass coverslips between $80-130 \mu \mathrm{m}$ of thickness (72198-11, EMS, USA). Substrates were plasma- 
cleaned (for $5 \mathrm{~min}$. on a clean glass petridish in a PDC-002 plasma-cleaner, Harrick Inc.) before preparation. A drop of medium was pipetted on top of the substrate at room temperature (RT), followed by a second drop after more than $5 \mathrm{~min}$. The substrate was rinsed with $2 \mathrm{ml}$ of DPBS. After detachment and washing of the cell-stock, a droplet of the cell suspension of typically $20 \mu \mathrm{l}(100-200$ cells/ $\mu \mathrm{l})$ was brought onto the partially wet and coated substrate. Cells settled for about 10 minutes. This step was carried out two to three times. After settlement, the samples were immersed in prewarmed nutrition medium, and incubated at $37^{\circ} \mathrm{C} / 5 \% \mathrm{CO}_{2}$ for up to 5 days. For C2C12-cells in a commercially available chamber (ibidi, Germany) cell suspension ( 40 cells $/ \mu \mathrm{l}$ ) was brought into a collagen IV-coated $\mu$-slide with a channel height of $200 \mu \mathrm{m}$. Sample was fixated the next day. The self-assembled glass chamber was formed using a second coverslip. A hole-punched Parafilm segment was placed on top. A droplet of DPBS was placed into the hole in the center. Next, the cell-containing glass coverslip was placed upside-down, and the Parafilm was locally heated forming a tight chamber. The chamber was finally sealed using nail polish.

Cryofixation: The samples were imaged by visible light phase contrast microscopy right before plunging. Substrates were then plunged and - when required - freeze-dried afterwards. All freeze-dried samples were transferred to silica gel-filled desiccators where they remained until shortly before the x-ray measurements.

Chemical fixation: Samples were fixated using pre-warmed $9 \%$ formaldehyde (252549, Sigma-Aldrich) in DPBS. All samples were washed with DPBS and stored in the refrigerator.

\section{Neonatal Rat Cardiac Tissue Cells}

Large parts are taken from (43).

Preparative work for micro-SAXS samples was performed by M. Eckermann (11) and Julia Scherber, preparative work for nano-SAXS samples was performed by Marten Bernhardt, Jan-David Nicolas, Susanne Hengst and Kristin Müller

Cultivation: Silicon nitride membranes (Silson, UK) of $1 \mu \mathrm{m}$-thickness were plasmacleaned (as above), coated with about $20 \mu \mathrm{l}$ coating solution $(0.08 \%$ gelatin (G7041, Sigma-Aldrich) and $0.5 \%$ fibronectin (F4759, Sigma-Aldrich) in sterile $\mathrm{H}_{2} \mathrm{O}$ ) and incubated for typically $2 \mathrm{~h}\left(37^{\circ} \mathrm{C}, 5 \% \mathrm{CO}_{2}\right)$. Disassembled neonatal rat cardiac tissue cell ${ }^{2}$ (NRCTCs) were resuspended in nutrition medium (DMEM-F12, 11039-021, Gibco; $10 \%$ FCS, 10270-106, Gibco; $1 \%$ pen./strep. 15140-122, Gibco) and then transferred onto the coated substrates either by placing a drop of cell suspension

2 obtained from Luther group; Max-Planck-Institute for Dynamics and Self-Organization, Am Faßberg, 37077 Göttingen 
or by submersing the substrate in a suspension-flooded well. In case of the former, cells were left to settle before wells were flooded with nutrition medium for overnight cultivation. Samples were incubated overnight, sometimes for several days, and monitored at different steps of the preparative procedure. Visible light phase contrast and fluorescence micrographs of entire samples were recorded before plunging and after freeze-drying.

Sample fixation and staining: Samples were fixated using $4 \%$ paraformaldehyde or $9 \%$ formaldehyde (252549, Sigma-Aldrich) in DPBS, permeabilized by Triton X-100, washed and stained with Hoechst 33342 and Phalloidin-A488 (Invitrogen, USA), following manufacturer protocols.

Cryofixation: Substrates were plunged and freeze-dried thereafter. All samples were transferred to desiccators filled with silica gel beads. In order to preserve the fluorescence signal, samples were stored in the dark until shortly before recording.

Alive samples: Cells were allowed to settle on the flat lid of the wet chamber (selfassembly cell from Silson, UK, top lid; see Sec.5.4 for details), transferred into a $15 \mathrm{ml}$ sealed vial filled with $\mathrm{CO}_{2}$-dependent nutrition medium (see above) and delivered on time to the beam line using a mobile incubator (without $\mathrm{CO}_{2}$ atmosphere). On arrival, the sample was transferred back to a stationary incubator $\left(37^{\circ}, 5 \% \mathrm{CO}_{2}\right)$ in order to recover buffer capacities. Shortly before recording, the cell containing top lid was transferred under a clean bench and the medium was replaced by degassed $\mathrm{CO}_{2}$-independent nutrition medium $\left(\mathrm{CO}_{2}\right.$-indep. Medium, 18045-054, Gibco; $10 \%$ FCS, 10270-106, Gibco; Pen/Strep, 15140-122, Gibco; 2 \% Glutamax, 35050-038, Gibco) before closing the chamber. The delivery process is also illustrated in Appendix Sec. 10.5 


\section{Section 3}

\section{Cellular SAXS: Model}

\section{independent Data Analysis \&}

\section{Fitting}

This section includes excerpts of (42) and (43).

Recent progress in x-ray optics and detector development have made possible the probing of a sample by scanning SAXS with subcellular real space resolution, so that local diffraction patterns can be assigned to cellular compartments (see Sec. 1 and Sec. 2). In order to interpret the structural details encoded in each pattern, one approach is to reduce every signal to a few directly accessible observables and then successively analyze an entire scan area in an automated scheme. In this manner, particular aspects of the scan can be quantified enabling further conclusions:

Sec. 3.1 addresses the automated analysis of anisotropic signals, Sec. 3.2 describes a procedure to correlate anisotropies to visible light fluorescence micrographs, and Sec. 3.3 presents an analysis tool for broad- $q_{\mathrm{r}}$-range radial intensity profiles $I\left(q_{\mathrm{r}}\right)$ as computed by azimuthal integration of each diffraction pattern. 


\subsection{Principal Component Analysis (PCA)}

The first approach introduced here is the principal component analysis (PCA) of anisotropic diffraction signals. Anisotropy originates from elongated, i. e. filamentous structures present at the illuminated spot such as the f-actin network of a cell. So far, there are a few tools available to quantify anisotropic SAXS patterns, all based on mathematically different approaches:

Weinhausen et al. (27) have introduced an automated ellipse fitting tool, which quantifies anisotropy based on the binarized and thresholded 2D diffraction signal, and has provided the means for the successful correlation of the visible light fluorescence micrograph of keratin-enriched carcinoma cells to scanning SAXS data. By using a set of parabolic refractive x-ray lenses, intense beam focusing could be achieved leading to a high real space resolution but also a radial symmetric empty beam profile, which is decisive for an adequate evaluation.

Priebe et al. (31) proposed another approach based on the (reweighted) azimuthal intensity profile, in the following denoted as the "streak finder" algorithm: Diffraction patterns are transformed into polar coordinates and normalized by the average radial intensity of the diffraction pattern, leading to a reweighted signal in a first step. The algorithm then calculates the corresponding azimuthal intensity profiles and determine the maximum of the reweighted signal, thus defining the orientation angle of the anisotropic signal (denoted as "streak"). The signal's anisotropy is quantified by considering the sum over the first six even Fourier components of the discrete series. In this way, cellular scans performed with x-ray instruments with an asymmetric angular empty beam profile (as e. g. caused by focusing with KB-mirrors) can also be reliably analyzed.

More, empirical and model based approaches by other research groups can be found in the work of Lichtenegger et al. (45), Makin et al. (46) and Bunk et al. (47).

PCA complements this toolbox of automated anisotropy analysis by a very simple but robust empirical approach reducing each diffraction pattern to a set of two eigenvectors, the so-called principal axes, and their corresponding eigenvalues representing the variances of the scattering signal along both axes. 


\subsubsection{Principal Proceeding}

The proceeding for principal component analysis on 2D diffraction patterns is as follows: Let $\mathrm{C}$ be the covariance matrix

$$
C=\left(\begin{array}{cc}
\operatorname{var}\left(q_{y}\right) & \operatorname{cov}\left(q_{y}, q_{z}\right) \\
\operatorname{cov}\left(q_{z}, q_{y}\right) & \operatorname{var}\left(q_{z}\right)
\end{array}\right)
$$

including the covariances

$$
\operatorname{cov}\left(q_{i}, q_{j}\right)=\frac{\sum_{m, n} I(m, n) \cdot\left[q_{i}(m, n)-\left\langle q_{i}\right\rangle\right] \cdot\left[q_{j}(m, n)-\left\langle q_{j}\right\rangle\right]}{\sum_{m, n} I(m, n)}
$$

with $\operatorname{var}\left(q_{i}\right)=\operatorname{cov}\left(q_{i}, q_{i}\right), I(m, n)$ denoting for the respective scattering intensities at pixel position $m, n \in \mathbb{N}$ on the (masked) detector, and $\left\langle q_{i}\right\rangle$ denoting for the expectation values

$$
\left\langle q_{i}\right\rangle=\frac{\sum_{m, n} I(m, n) \cdot q_{i}(m, n)}{\sum_{m, n} I(m, n)} .
$$

with $i=y, z$. Solving the eigenvalue problem

$$
C \cdot \vec{u}_{k}=\lambda_{k} \cdot \vec{u}_{k}
$$

then leads to a set of eigenvectors $\vec{u}_{k}$ together with their corresponding eigenvalues $\lambda_{k}, k \in[1,2]$. The eigenvectors $\vec{u}_{k}$ are denoted as the principal axes and establish a new orthogonal base in reciprocal space. The eigenvalues $\lambda_{k}=\sigma_{k}^{2}$ equal the variances along both directions and assign the principal axes to a "line of best fit" and a "line of worst fit" (48, 49). For further processing, both eigenvectors are scaled to unity $\vec{e}_{k}=\vec{u}_{k} /\left\|\vec{u}_{k}\right\|$ and sorted by the relative variance $\lambda_{k}$. The largest variance $\lambda_{\mathrm{pa}}=\max \left(\lambda_{1}, \lambda_{2}\right)$ belongs to the eigenvector aligned along the anisotropic signal, thus defining the anisotropy orientation angle $\gamma_{\mathrm{pa}}$. The corresponding structure orientation angle $\theta_{\mathrm{pa}}$ is derived by

$$
\theta_{\mathrm{pa}}=\gamma_{\mathrm{pa}}+90^{\circ}
$$

This scheme can be applied on an entire scan area, characterizing the anisotropy for each scan point $(a, b) \in \mathbb{N}^{2}$ in an automated manner. 
Furthermore, a unitless order parameter

$$
\omega_{\mathrm{pa}}(a, b)=\frac{\left|\lambda_{1}(a, b)-\lambda_{2}(a, b)\right|}{\lambda_{1}(a, b)+\lambda_{2}(a, b)}
$$

quantifies the anisotropic character of a diffraction signal.

Note, that the variances $\lambda_{k}(a, b)$, obtained in units of $\mathrm{nm}^{-2}$, can be converted in the respective standard deviations along the corresponding directions by

$$
\sigma_{k}(a, b)=\sqrt{\lambda_{k}(a, b)}
$$
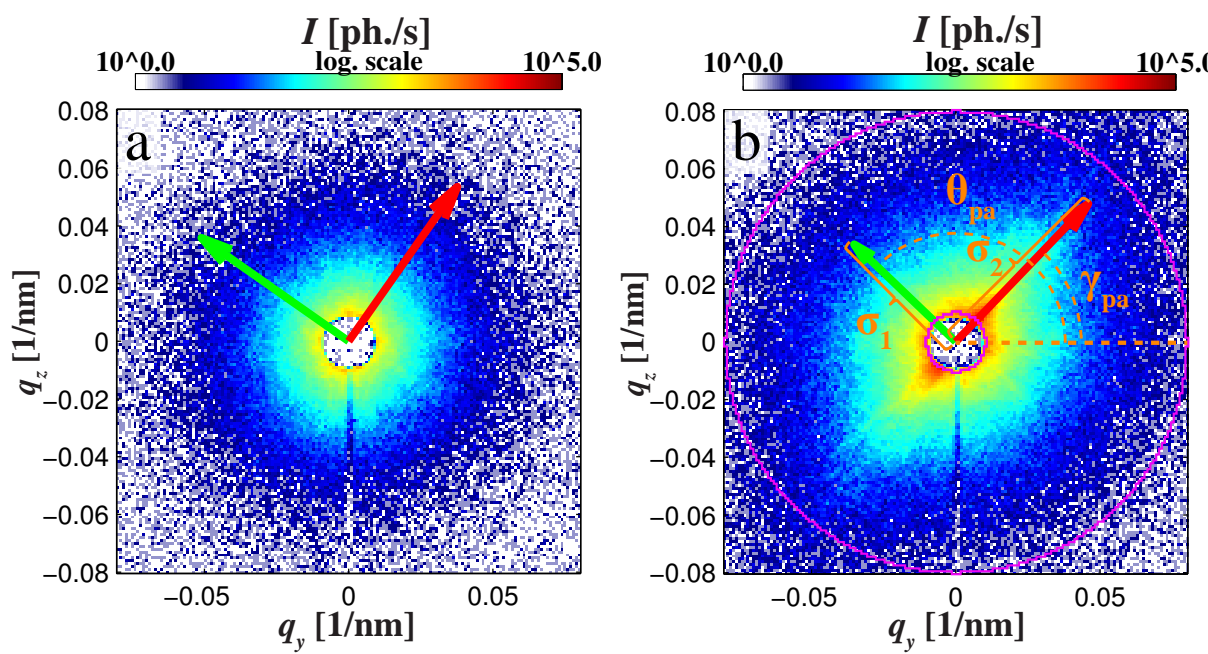

Figure 3.1: Two representative diffraction patterns showing an (a) isotropic and an (b) anisotropic behavior including the respective rescaled principal axes $\vec{l}_{k}$ (green and red arrows). For presentation purposes, both axes were multiplied by an arbitrary scaling factor, while keeping the aspect ratio unaffected. Figure and caption adapted from [42).

Hence, the standard deviations are measured in $\mathrm{nm}^{-1}$, that is equivalent to the units in reciprocal space, which makes $\sigma_{k}(a, b)$ appropriate scaling factors,

$$
\vec{l}_{k}(a, b)=\sigma_{k}(a, b) \cdot \vec{e}_{k}(a, b)
$$

as well as the aspect ratio

$$
A_{\mathrm{r}}(a, b)=\frac{\left|\vec{l}_{\mathrm{pa}}(a, b)\right|}{\left|\vec{l}_{\mathrm{k} \neq \mathrm{pa}}(a, b)\right|}
$$

between both axes an appropriate depiction of the anisotropic strength of the signal. 
Fig. 3.1 exemplarily shows two cellular diffraction signals as recorded by micro-SAXS scanning with a rather isotropic, see $(a)$, and an anisotropic pattern, see $(b)$, together with the corresponding rescaled principal axes $\vec{l}_{k}(a, b)$. While the isotropic signal leads to an aspect ratio $A_{\mathrm{r}}(a, b)$ close to one, it becomes relatively large in the anisotropic case.

Similar to the ellipse fitting tool of Weinhausen et al. (27), this procedure in principle requires a radial symmetric empty beam signal and a gap-free, homogeneous detection area. However, depending on the $q$-range and the experimental setup, detection areas might be interrupted by inter-modular gaps of the detector which causes a lack of data for certain $\left(q_{y}, q_{z}\right)$-tuples, while parasitic scattering effects responsible for an asymmetric empty beam profile can hinder a further data analysis. Nevertheless, as will be shown in Sec.5.3. PCA turned out to be sufficiently robust even when dealing with these less ideal conditions. As regards the example shown in Fig. 3.1 diffraction patterns of a Pilatus $2 \mathrm{M}$ detector (50) were restricted to the center module around the primary beam, before applying a ring-shape mask (indicated as pink discs) defining the evaluation area.

\subsubsection{First Applications on Model \& Real Data}

In order to illustrate the general behavior of PCA with respect to the main characterizing observables $\gamma_{\mathrm{pa}}$ and $\omega_{\mathrm{pa}}$ as described above, basic model patterns are evaluated: Fig. 3.2 (a) shows some selected input patterns, that are defined on the basis of a (normalized) 2D Gaussian profile along the two directions $q_{y}$ and $q_{z}$ in reciprocal space following the expression

$$
\begin{aligned}
I\left(q_{y}, q_{z}\right) & =\frac{\frac{1}{2 \pi \sigma_{q_{y}} \sigma_{q_{z}}} \cdot \mathrm{e}^{-\left(\frac{q_{y}^{2}}{2 \sigma_{q_{y}}^{2}}+\frac{q_{z}^{2}}{2 \sigma_{q_{z}}^{2}}\right)}}{\frac{1}{2 \pi} \cdot \max \left(\mathrm{e}^{-\frac{q_{y}^{2}}{2 \sigma_{q_{y}}^{2}}} / \sigma_{q_{y}}\right) \cdot \max \left(\mathrm{e}^{-\frac{q_{z}^{2}}{2 \sigma_{q_{z}}^{2}} / \sigma_{q_{z}}}\right)} \\
& =\mathrm{e}^{-\left(\frac{q_{y}^{2}}{2 \sigma_{q_{y}}^{2}}+\frac{q_{z}^{2}}{2 \sigma_{q_{z}}^{2}}\right)} .
\end{aligned}
$$

This profile is then successively rotated, see (1) "rotating Gaussian profile", expanded evenly towards both directions, see (2) "growing Gauss ring", or expanded unevenly by elongating towards one direction, while keeping static the other, see (3) "expanding Gaussian profile”. 
(1) In case of the "rotating Gaussian profile" the corresponding rescaled eigenvectors $\vec{l}_{k}$ follow the direction of the anisotropic portion (pink arrows). However, the order parameter $\omega_{\text {pa }}$ remains close to 1 since both variances $\lambda_{k}$ (with respect to the new coordinate system established by $\vec{u}_{k}$ ) do not change. This can be further illustrated in a respective mapping of PCA results, see $(b)$, showing the anisotropy orientation angle $\gamma_{\mathrm{pa}}$ (red lines) and the anisotropy parameter $\omega_{\mathrm{pa}}$ (color code). (2) Expanding, but fully isotropic patterns ("growing Gauss ring") do not influence the values of either of these observables. (3) In case of regarding an "expanding Gaussian profile”, PCA first calculates decreasing values for $\omega_{\text {pa }}$ until reaching isotropy, where $\omega_{\text {pa }}$ equals 0 . Here, $\gamma_{\text {pa }}$ flips in an instant and $\omega_{\mathrm{pa}}$-values successively rise again.

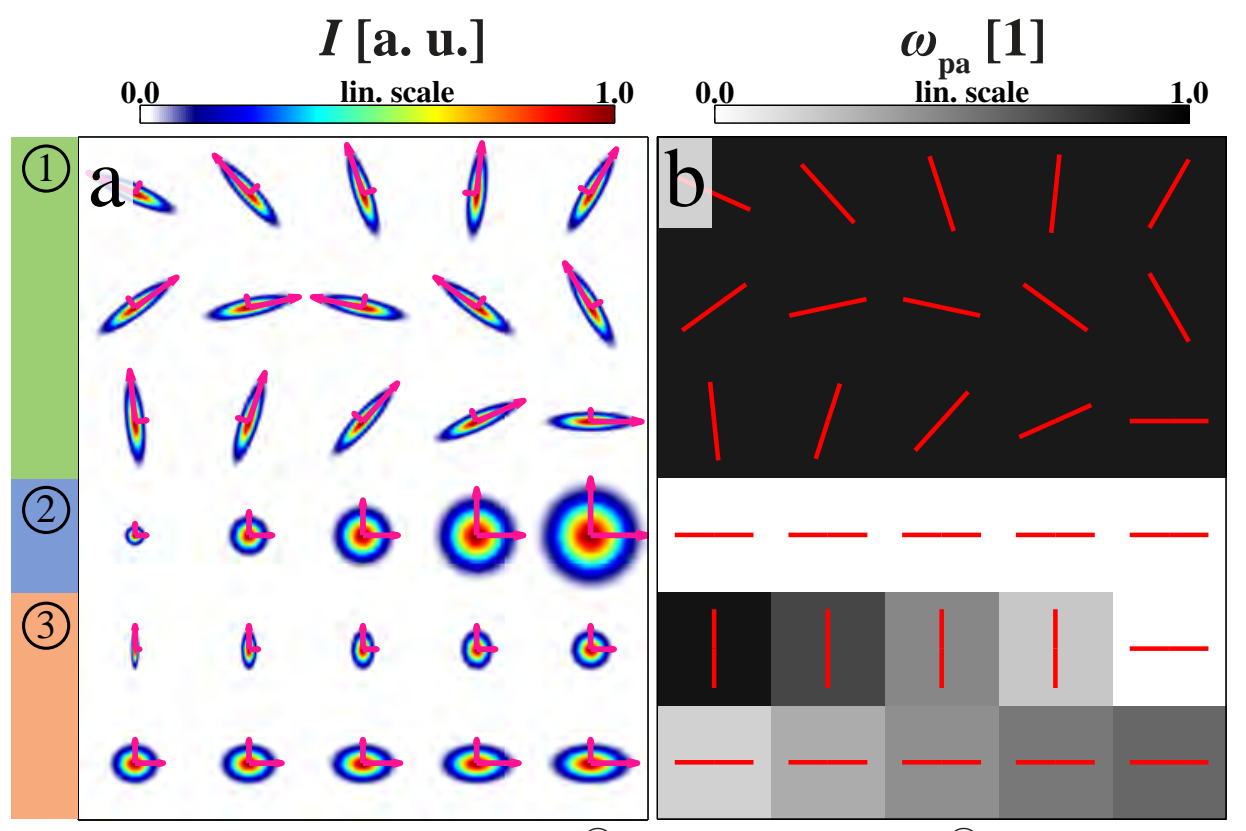

Figure 3.2: (a) Selected input patterns comprising (1) a rotating Gaussian profile, (2) a growing Gauss ring and (3) an expanding Gaussian profile together with the respective rescaled principal axes $\vec{l}_{k}$ as defined in Eq. 3.8 Results are simulated based on experimental parameters of the micro-SAXS setup $\left(E_{\mathrm{ph} .}=8.7 \mathrm{keV}\right.$, sample-detector-distance: $\left.7.5 \mathrm{~m}\right)$, here showing data up to $q_{\max }=0.097 \mathrm{~nm}^{-1}$. (b) Corresponding PCA output mapping, comprising the anisotropy orientation angle $\gamma_{\mathrm{pa}}$ (red lines) and the order parameter $\omega_{\mathrm{pa}}$ (color code).

Fig. 3.3 shows a first application of PCA on a real dataset, here on the example of a freeze-dried murine myoblast, further illustrating the analytic potential of this tool: Fig. 3.3 (a) shows the x-ray dark field (together with the OAV-micrograph, inset) revealing a relatively high scattering intensity in the center of the cell. Beam dimensions that are 
on the same order of the cell size blur the real-space image so that the contour of nucleus \& the cellular cortex cannot be identified with adequate precision.

A clean empty beam signal allows an adequate tracking of local anisotropies by PCA; Fig. $3.3(b)$ shows the order parameter $\omega_{\mathrm{pa}}(a, b)$ in color code and the structure orientation angles $\theta_{\mathrm{pa}}(a, b)$, thresholded to local $\omega_{\mathrm{pa}}$-values of above 0.1 , as white lines. In agreement with $(c)$ showing neighboring diffraction patterns of a selected region, results indicate a rather pronounced anisotropic character of diffraction signals throughout the entire cell with a maximum in the central region.

In order to further characterize the overall scattering behavior of the cell, an averaged (background-corrected) 2D diffraction pattern is computed on the basis of the regions marked in $(a)$. PCA on this averaged pattern leads to the two principal axes (indicated as black dashed lines) with axis 1 (at angle $\gamma_{1}$ ) directing along and axis 2 (at angle $\gamma_{2}$ ) standing perpendicular to the anisotropic signal, the latter indicating the (mean) structure orientation of the averaged signal.

The respective standard deviations $\sigma_{1}=1.9 \cdot 10^{-2} \mathrm{~nm}^{-1}$ and $\sigma_{2}=1.3 \cdot 10^{-2} \mathrm{~nm}^{-1}$ facilitate quantifying of the average scattering distribution in a model-free manner. The results correspond to real space lengths of $d_{1}=\pi / \sigma_{1}=169 \mathrm{~nm}$ and $d_{2}=\pi / \sigma_{2}=238 \mathrm{~nm}$ (following Eq. 2.10). For a further analysis towards the radial intensity decays two sectors are defined ranging $\pm 10^{\circ}$ around $\gamma_{1}$ and $\gamma_{2}$. The corresponding radial profiles $\left.I\left(q_{\mathrm{r}}\right)\right|_{\gamma_{k} \pm 10^{\circ}}$ are obtained by azimuthal integration over the sectors leading to results as exemplarily shown for axis 1 , see $(e)$. Orange and gray curves denote the (pure) cellularand background-diffraction signal; the blue curve marks the respective backgroundcorrected cellular profile and can be described by a (simple) power law decay following Eq. 2.5. The results for both axes are listed in Tab. 3.1 revealing a slight but significant difference of $\left|b_{1}-b_{2}\right| \approx 0.2$ in the exponents.

\begin{tabular}{cllllll}
\hline axis & $\boldsymbol{a}$ & $\boldsymbol{b}$ & $\boldsymbol{c}$ & $\mathbf{r}^{2}$ & $\sigma$ & $\boldsymbol{d}$ \\
& {$[(\mathrm{ph} . \cdot \mathrm{nm}) / \mathrm{s}]$} & {$[1]$} & {$[\mathrm{ph} . / \mathrm{s}]$} & {$[1]$} & {$[1 / \mathrm{nm}]$} & {$[\mathrm{nm}]$} \\
\hline 1 & $2.0 \cdot 10^{-4}$ & -3.95 & $2.7 \cdot 10^{-2}$ & 0.97 & $1.9 \cdot 10^{-2}$ & 169 \\
\hline & $1.2 \cdot 10^{-4}$ & -3.74 & $3.1 \cdot 10^{-2}$ & 0.96 & $1.3 \cdot 10^{-2}$ & 238 \\
\hline
\end{tabular}



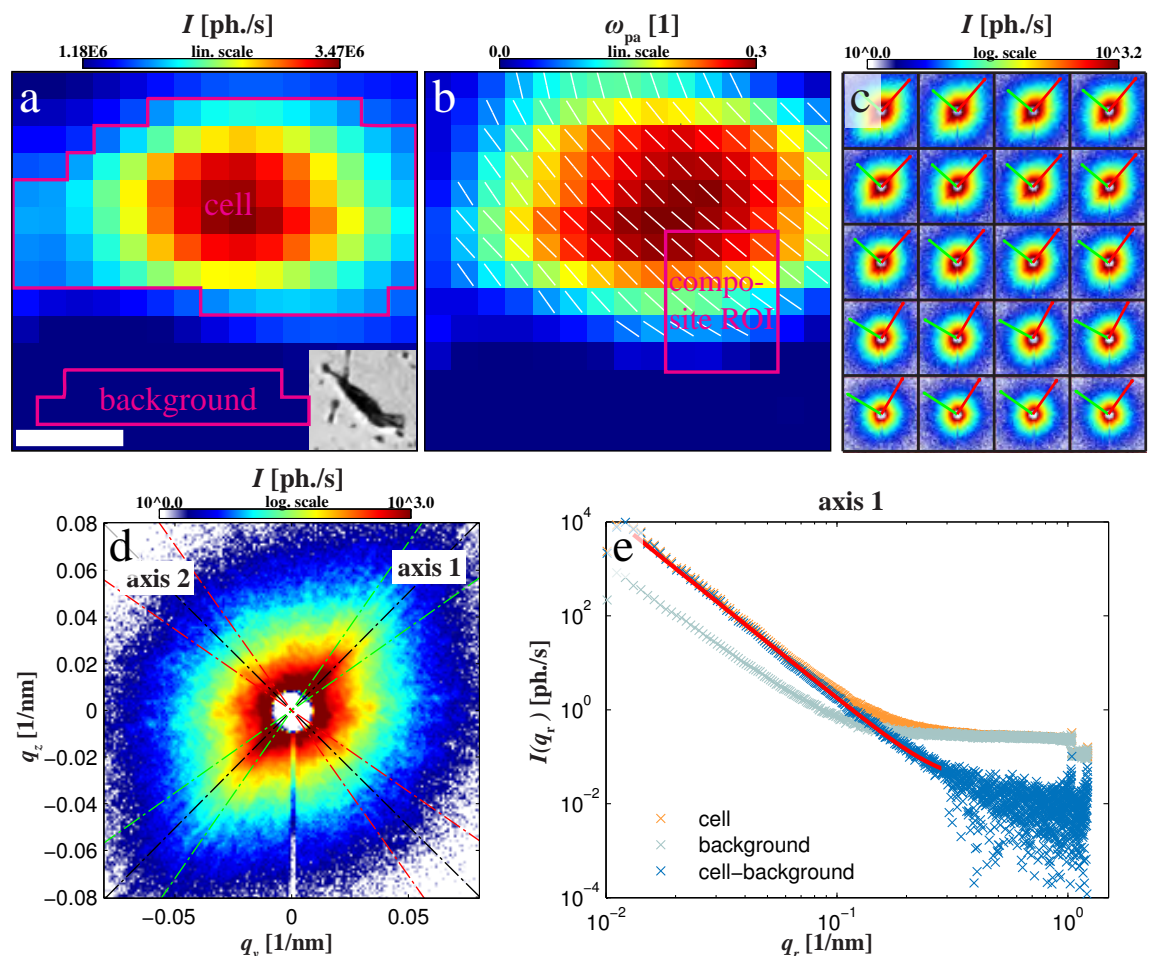

Figure 3.3: (a) X-ray dark field of a micro-SAXS scan performed on a freeze-dried murine myoblast. inset: OAV micrograph of the cell right before scanning. Scale bar: $20 \mu \mathrm{m}$. (b) PCA-results showing the order parameter $\omega_{\mathrm{pa}}$ (color code) and structure orientation angles $\theta_{\mathrm{pa}}$ of diffraction patterns with $\omega_{\mathrm{pa}}>0.1$ (white lines). (c) Diffraction patterns of directly neighboring scan points of the region marked in (b) including the rescaled eigenvectors $\vec{l}_{k}$ (see Eq. 3.8) (red \& green arrows). Each diffraction pattern is cropped to the central region around the primary beam $\left(q_{\mathrm{r}}<0.11 \mathrm{~nm}^{-1}\right)$. (d) Averaged (background-corrected) $2 D$ diffraction pattern considering the areas marked in a. (e) Radial intensity profiles regarding averaged diffraction data $\pm 10^{\circ}$ around axis 1. The decay of the background-corrected signal is described by a power law function (see Eq.2.5. Figure and caption adapted from [42). 


\subsubsection{Multiple Streak Analysis}
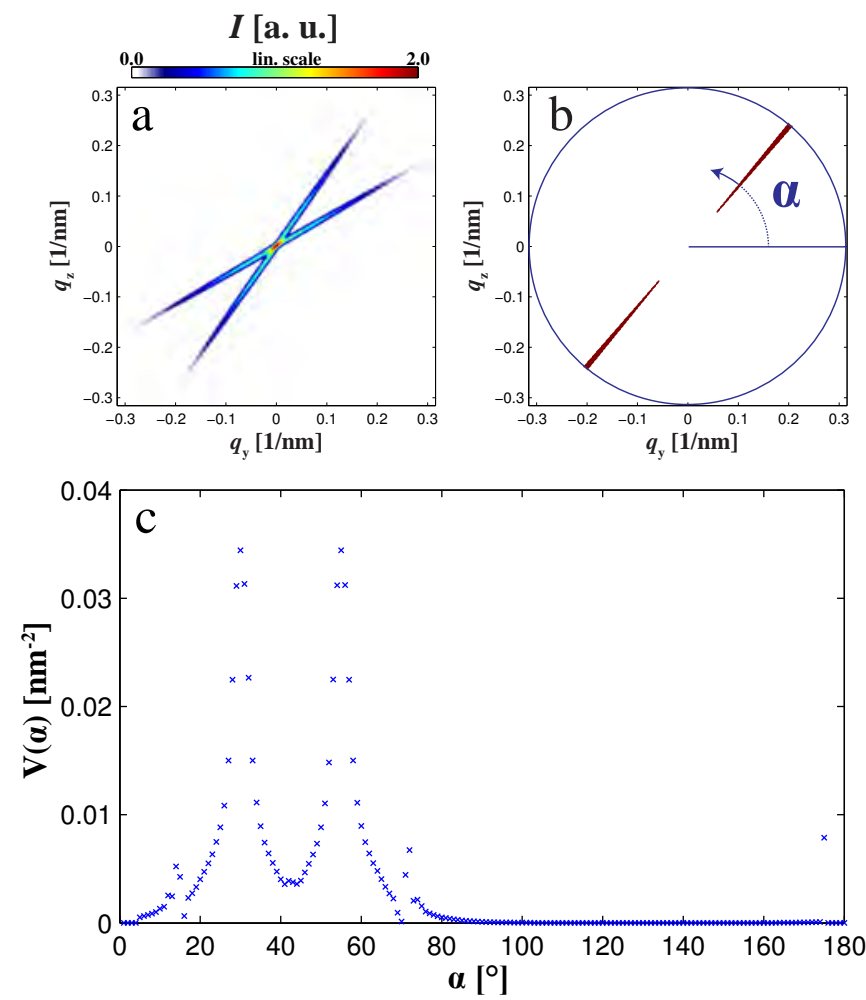

Figure 3.4: (a) Double Gaussian signal using the typical parameters of the GINIX setup ( $E_{\mathrm{ph} .}=7.9 \mathrm{keV}$, sample detector distance $5.07 \mathrm{~m}$ ) (b) A "rotating mask" enables a successive evaluation of the diffraction signal, with $\alpha \in\left[0^{\circ}, 180^{\circ}\right]$. (c) Plot of the variance $V$ as a function of the evaluation angle $\alpha$.

Multiple anisotropic portions within a scattering signal might originate from crossovers of filaments (see Sec.3.1.4, parasitic scattering effects established by an unclean empty beam profile or even the formation of ice crystals on cryoprotected samples (see Sec. 4.5. Especially the last two examples are critical, since they can lead to a (systematic) misinterpretation of diffraction data. As will be further discussed in the following Sec. 3.1.4 PCA will determine principal axes that lie somewhere in between the anisotropic portions for all of those cases.

However, identifying and possibly further dissecting such signals can be addressed by just applying a slight modification to the evaluation mask: Fig. 3.4 (a) shows a model double Gauss-streak profile directing towards $\gamma_{\text {Gauss } 1}=30^{\circ}$ and $\gamma_{\text {Gauss } 2}=55^{\circ}$. (b) $\mathrm{A}$ narrow-cut and successively rotating propeller-shaped evaluation mask (opening an- 
gle: $2^{\circ}$, rotating step size: $\left.1^{\circ}, \alpha \in\left[0^{\circ}, 180^{\circ}\right)\right)$ enables the determination of the respective variances $V(\alpha)$ as a function of $\alpha$. Fig. 3.4 (c) shows the results for all variances $V(\alpha)$ reaching two global maxima around the orientation angles $\gamma_{\text {Gauss } k}$ of each single streak. Depending on the signal-to-noise ratio of a particular scan, these plots can be thresholded up to a significant value for $V(\alpha)$ to determine the number and position of multiple anisotropic signals.

\subsubsection{Comparison of Anisotropy Analysis Tools}

In order to compare the different mechanisms of the ellipse fitting tool (by Weinhausen et al. (27)), the streak finder (by Priebe et al. (31)) and the PCA (42), all analysis tools are applied on a nano-SAXS dataset as obtained from a frozen-hydrated sample of an in vitro f-actin suspension (data taken from Töpperwien et al. (32)). Fig.3.5(a) shows the composite image of a selected region including a cross-over of actin filaments: Diffraction patterns reveal pronounced single and multiple anisotropies as well as a rather isotropic background signal. Fig.3.5 (b) shows the x-ray dark field of the area including all structure orientation angles as computed by the ellipse fitting tool (red lines), the streak finder algorithm (blue lines) and the PCA (with structure orientation angles $\theta_{\mathrm{pa}}$, green lines).

It becomes apparent, that the structure orientation of all three algorithms are in good agreement for diffraction patterns showing a pronounced single anisotropic signal. Contrarily, results differ significantly in case of multiple anisotropies as well as (the relatively weak) isotropic background signals.

Consistent with the corresponding algorithm that is applied, the anisotropic character of each diffraction pattern is quantified by the eccentricity $\varepsilon$ of the fitted ellipse (as further detailed in (27), results: see $(c)$ ), the anisotropy parameter $\breve{S}$ (as further detailed in (31), results: see $(d)$ ), or the order parameter $\omega_{\mathrm{pa}}$ (as defined in Eq. 3.6. results: see (e)). Orientation angles are thresholded to values of $\varepsilon>0.63, \breve{S}>0.05$ and $\omega_{\mathrm{pa}}>0.04$, respectively.

All algorithms detect anisotropic signals accurately well, but judge the anisotropic portions in very different ways: The ellipse fitting tool processes a binarized signal of single (background-corrected) diffraction pattern considering photon counts in each single pixel that lie beyond a manually defined threshold (here $\geq 22 \mathrm{ph}$./s for each detector pixel as determined on the median-filtered detector image). This approach flattens the signal thus adding more weight to weak, but still noticeable scattering intensities, which makes this approach sensitive for auxiliary anisotropic scattering effects. A sub- 
sequent ellipse fit promotes tracing of the displacement (i. e. center of mass) of all binarized pixels in a straight forward manner.

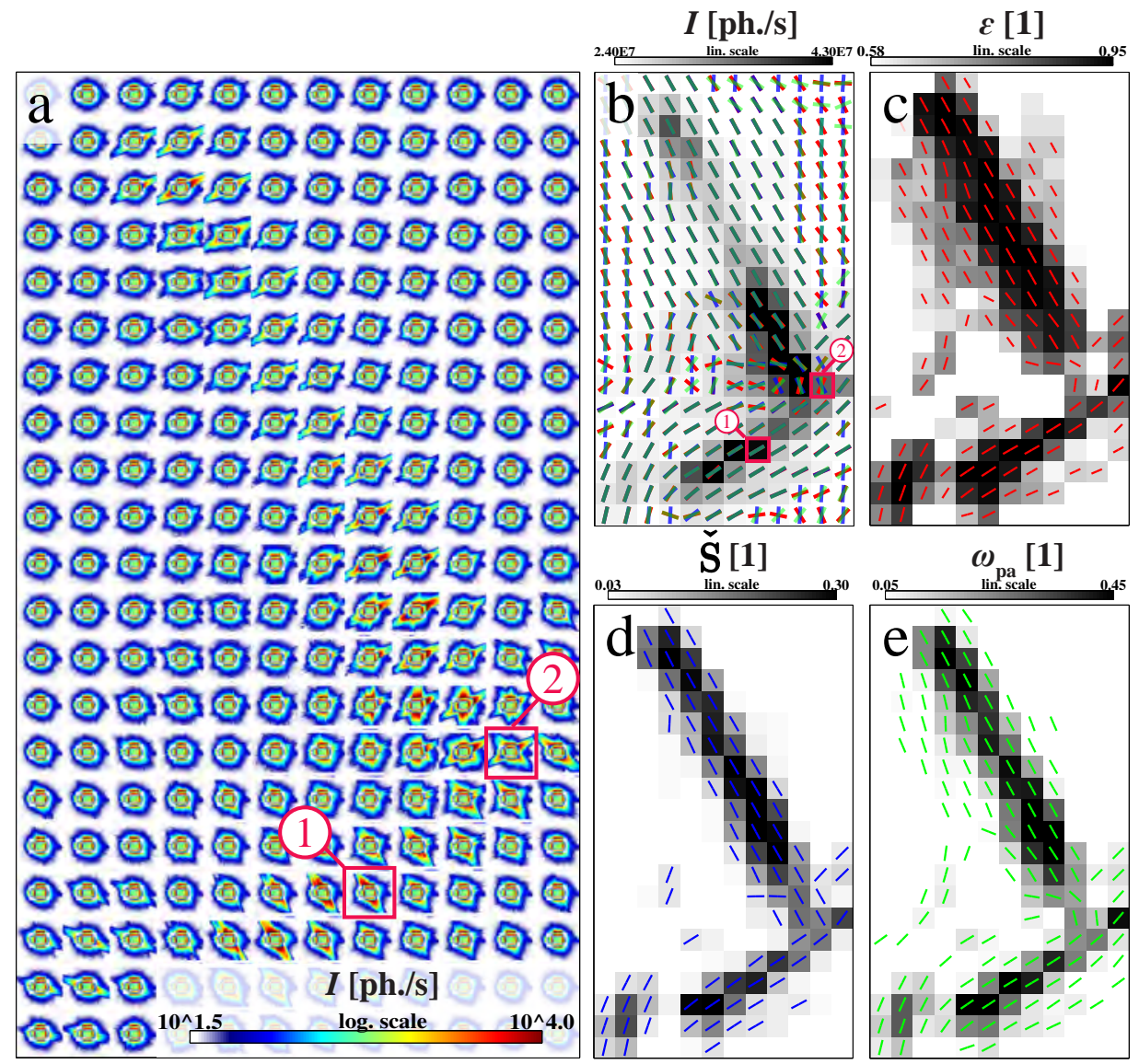

Figure 3.5: (a) Composite image of a nano-SAXS scan on frozen-hydrated in vitro f-actin (data from Töpperwien et al. [32) showing $2 D$-diffraction patterns with respect to the sample position. (b) X-ray dark field including all structure orientation angles as computed by (c) the ellipse fitting tool 27) (red lines), (d) the streak finder algorithm [31] (blue lines) and (e) the PCA (green lines). The color code in (c-e) denotes for the eccentricity $\varepsilon$, the anisotropy parameter $\breve{S}$ and the order parameter $\omega_{\mathrm{pa}}$. Two positions marked by (1) \& (2) are singled out for close inspection, see Fig. 3.6 and text.

The streak finder tool analyzes the (reweighted) azimuthal intensity profile of a signal defining its global maximum as the structure orientation angle and thus making it very robust against any influences caused by asymmetric empty beam shapes or parasitic scattering effects. The corresponding anisotropy parameter $\check{S}$ is computed from the first six even components of the profile in Fourier space. 
Finally, PCA determines the principal axes and variances considering the covariance matrix of each diffraction patterns as further detailed in Sec.3.1.1.

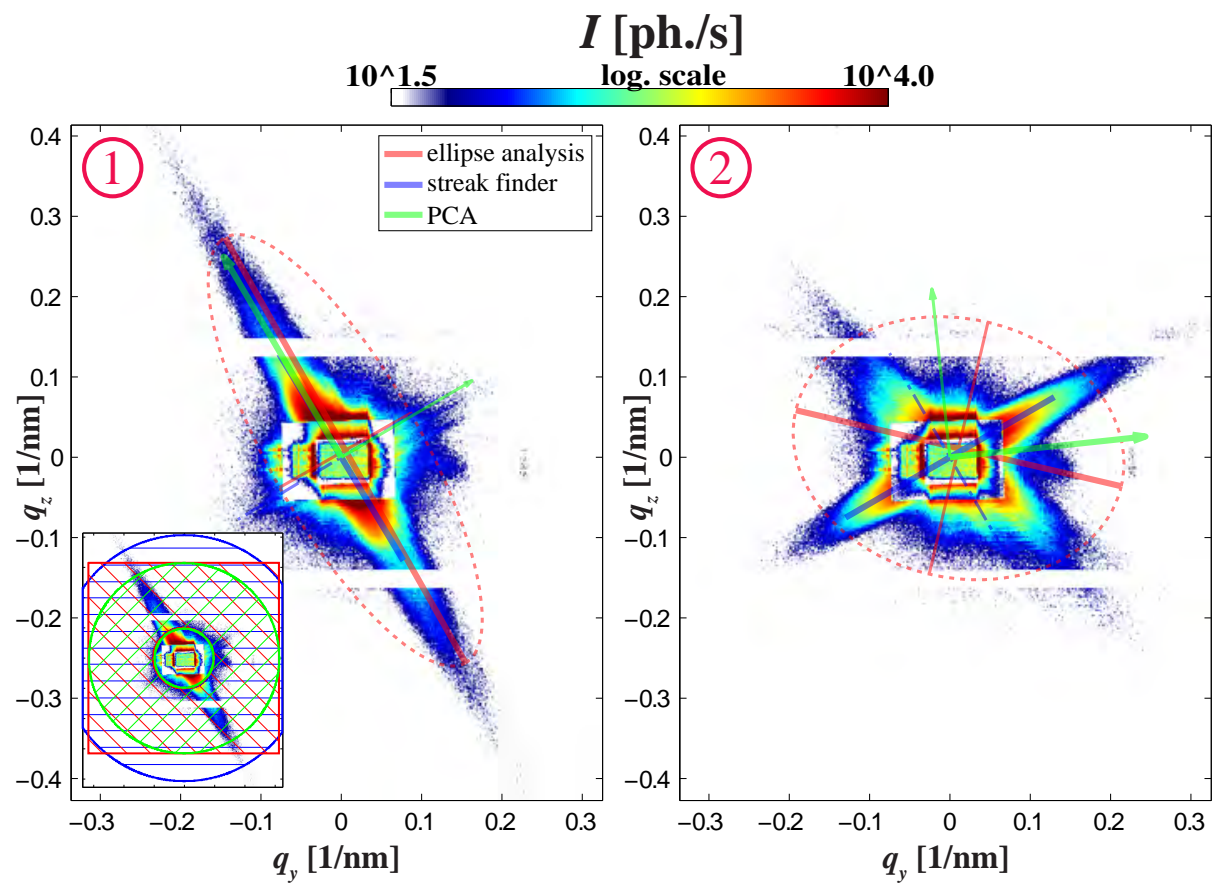

Figure 3.6: (1) Single streak diffraction pattern analyzed with the ellipse fitting tool (red), the streak finder algorithm (blue) and the PCA (green). Inset: Evaluation areas of the corresponding algorithm applied on each diffraction pattern. (2) Multiple streak diffraction pattern.

In order to illustrate these differences further, Fig. 3.6 shows the diffraction patterns of spots (1) \& (2) (at positions marked in Fig. 3.5 (a-b)) comprising a signal with a pronounced single anisotropic portion, see (1), and one with multiple anisotropic portions, see (2). All three algorithms are applied, considering the respective evaluation areas as marked in (a), (see inset, hatched areas). The corresponding results are indicated by colored semitransparent lines, including (i) the major and minor axis of the ellipse (thick \& thin red lines), (ii) the global maximum of the reweighted azimuthal plot together with the intended orthogonal structure orientation (thick $\&$ thin blue lines) as well as (iii) the rescaled principle axis $\vec{l}_{k}$ (thick \& thin green arrows). Note, that when comparing these results to Fig. 3.5 the line orientation of the framed pixels in Fig. 3.5 (showing the structure orientation) correspond to an orientation that is $90^{\circ}$-shifted to the anisotropic portion of the diffraction patterns. The line orientation is thus directing along the minor axis of the ellipse (thin red line), 
an intended orientation according to streak finder algorithm (thin dashed blue line) and the "line of worst fit" (thin green arrow), respectively.

(1) In agreement with previous observations, the results coincide to a large degree in the case of the single (pronounced) anisotropic signal. Notably, the ellipse center is slightly shifted to the manually defined primary beam position (estimated 'by eye') as considered for the streak finder- and PCA algorithm. (2) Analysis of a multiple anisotropic signal leads to quite distinct results, again illustrating the significant differences among all analysis tools in terms of data processing.

In conclusion, all three algorithms complement each other very well, enabling the elucidation of different aspects of a scan. 


\subsection{Comparative Analysis of Fluorescence Microscopy \& Scanning SAXS}

Towards a further analysis of the cause of anisotropic diffraction signals, one possible approach is to correlate $\mathrm{x}$-ray data of a sample region with data obtained from an entirely different but more established (imaging) technique. In this work, fluorescence microscopy has played an important role in order to identify the local structure of the actin cytosceleton of a particular cell but at the same time has triggered the desire for a comparative analysis tool contrasting visible light fluorescence and scanning SAXS results in a quantitative manner.

However, for a pixelwise comparison, the fluorescence data have to undergo an additional analysis step as well in order to obtain the filament orientation in an output matrix that assigns the filament angle to the relative position within a cellular area. For this purpose, micrographs are analyzed using the filament sensor, as published by Eltzner et al. (51), in order to track the most significant filaments that are above a manually defined threshold. This results in an output file comprising pixel-specific values for the filament orientation angle, in the following denoted as $\vartheta_{\mathrm{fs}}(\tilde{y}, \tilde{z}) \in[0,180)$ for each pixel position $(\tilde{y}, \tilde{z}) \in \mathbb{N}^{2}$ on the fluorescence image.

However, in usual cases, the pixel size of the micrograph does not correspond to the step size chosen for a SAXS scan. Post processing, which was kindly conceived, developed and implemented by Dr. Benjamin Eltzner, enabled adjustment of the data output file by segmenting the fluorescence micrograph into square blocks $B(a, b)$ of size $L \times L$, the equivalent to the scanning step size $\Delta$ for the x-ray scan. His algorithm then calculates an intrinsic mean $\mu_{\text {int }}(a, b)$ and the variance $V_{n}\left(\mu_{\text {int }}(a, b)\right)$ for each block following the work of Hotz et al. (52):

$$
\begin{aligned}
f_{(a, b)}(\theta) & :=\sum_{(\tilde{y}, \tilde{z}) \in \tilde{B}(a, b)} \min \left(\left|\theta-\vartheta_{\mathrm{fs}}(\tilde{y}, \tilde{z})\right|, 180^{\circ}-\left|\theta-\vartheta_{\mathrm{fs}}(\tilde{y}, \tilde{z})\right|\right)^{2} \\
\theta_{\mathrm{fs}}(a, b):=\mu_{\mathrm{int}}(a, b) & =\underset{\theta \in\left[0^{\circ}, 180^{\circ}\right)}{\operatorname{argmin}} f_{(a, b)}(\theta) \\
V_{n}\left(\mu_{\mathrm{int}}(a, b)\right) & =\min _{\theta \in\left[0^{\circ}, 180^{\circ}\right)} f_{(a, b)}(\theta) /(N(a, b)-1)
\end{aligned}
$$

with the cardinality $N(a, b)=|\tilde{B}(a, b)|$ of the set of filament containing pixels $\tilde{B}(a, b)=$ $\left\{(\tilde{y}, \tilde{z}) \in B(a, b), \vartheta_{\mathrm{fs}}(\tilde{y}, \tilde{z}) \geq 0\right\}$ within a block, and the mean orientation angle $\theta_{\mathrm{fs}}(a, b)$. 
A polarization parameter $\omega_{\mathrm{fs}}(a, b)$ quantifies the standard deviation within a block:

$$
\omega_{\mathrm{fs}}(a, b)=1-\frac{\sqrt{V_{n}\left(\mu_{\mathrm{int}}(a, b)\right)}}{90^{\circ} / \sqrt{3}}
$$

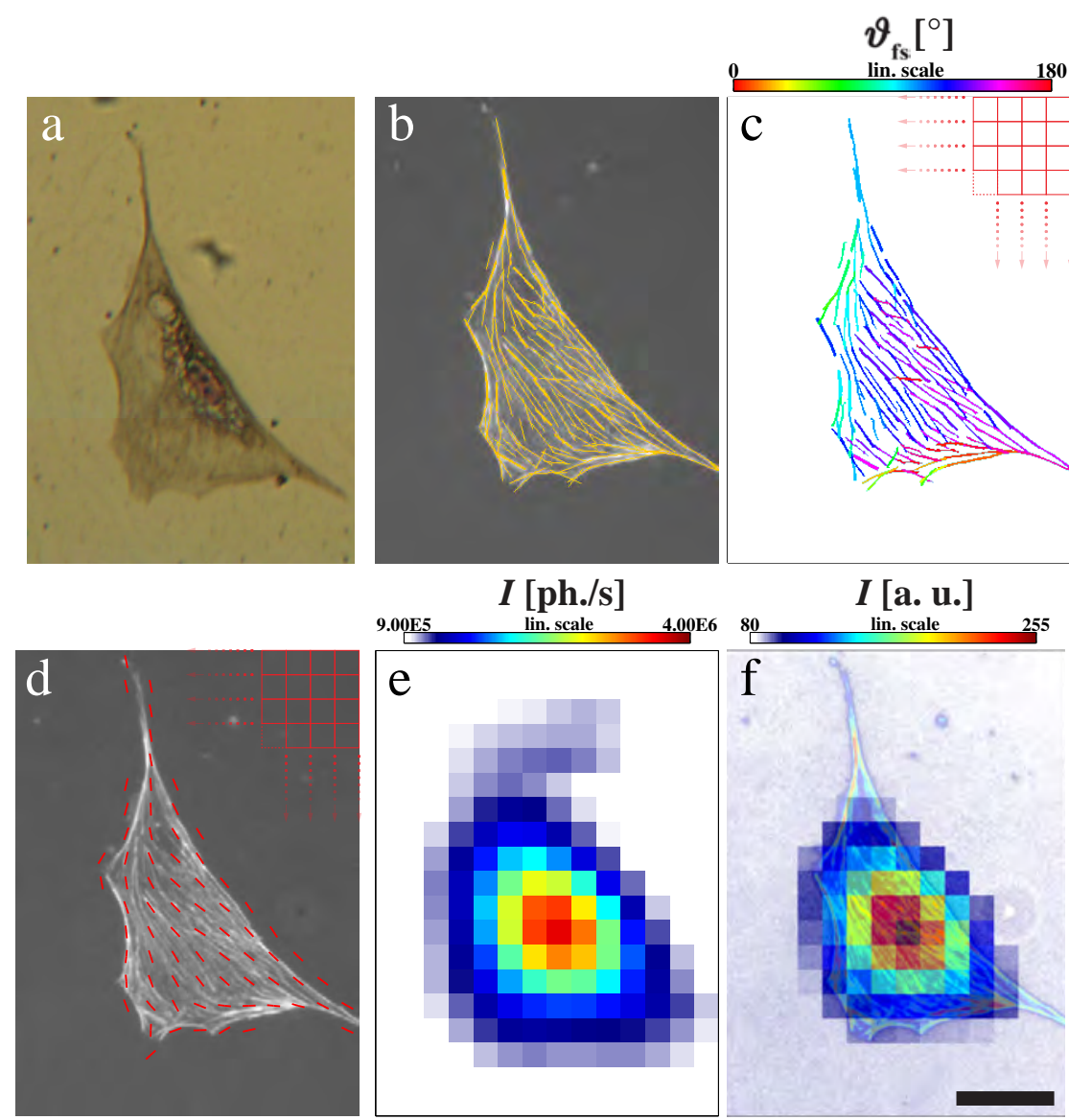

Figure 3.7: (a) OAV-micrograph of a freeze-dried NRCTC. (b) Fluorescence micrograph of the cell after freezedrying including all tracked filaments (yellow lines). (c) Results for $\vartheta_{\mathrm{fs}}(\tilde{y}, \tilde{z})$ in a color plot. Within white areas, no trackable filament is present. (d) Fluorescence micrograph including the binned orientation angles $\theta_{\mathrm{fs}}(a, b)$ for each block (red lines). (e) $x$-ray dark field of the particular cell. $(f)$ Superimposition image considering the $x$-ray dark field (semitransparent top layer) and the fluorescence micrograph (non-transparent bottom layer). Scale bar: $40 \mu \mathrm{m}$. Figure and caption adapted from [43). 
$\chi_{\mathrm{fs}}(a, b)$ measures the amount of orientation-containing pixel:

$$
\chi_{\mathrm{fs}}(a, b)=\frac{N(a, b)}{L^{2}}
$$

Fig. 3.7 3.8 further illustrate this procedure showing the major results of each step:

Fig. $3.7(a-b)$ shows the OAV-micrograph of a freeze-dried NRCTC on an ultrathin SiRNwindow right before recording, see $(a)$, together with the visible light fluorescence image of the cell after freeze-drying, see $(b)$. The algorithm assigns local orientation angles $\vartheta_{\mathrm{fs}}(\tilde{y}, \tilde{z})$ of all trackable filaments (yellow lines) to the respective pixel positions, see $(c)$. Pixels, that contain no trackable filament, are set to an invalid orientation $\vartheta_{\mathrm{fs}}(\tilde{y}, \tilde{z})=-1^{\circ}$ and are depicted in white.

Segmentation of the fluorescence image (indicated as red square blocks) result in the mean orientation angles $\theta_{\mathrm{fs}}(a, b)$ as shown in $(d)$ (red lines) and requires smoothly devisible edge lengths $\max (\tilde{y})$ and $\max (\tilde{z})$ of the fluorescence micrograph holding the condition

$$
\max (\tilde{y}) \bmod L=0 \wedge \max (\tilde{z}) \bmod L=0 .
$$

The block size $L \in \mathbb{N}$ in $[\mathrm{px}]$ is defined by the pixel size $\xi_{\text {fluo }}$ of the fluorescence micrograph (in $[\mathrm{px} / \mu \mathrm{m}]$, i. e. the pixel density $1 / \xi_{\text {fluo }}$ in $[\mu \mathrm{m} / \mathrm{px}]$ ) and the step size $\Delta$ as chosen for the $\mathrm{x}$-ray scan

$$
L=\left\lceil\frac{\Delta}{\xi_{\text {fluo }}}\right\rfloor .
$$

Brackets denote rounding the argument to the nearest integer value. The respective residual amount

$$
\delta_{L}=\left(\frac{\Delta}{\xi_{\text {fluo }}}\right) \bmod L
$$

is minimized by rebinning of the input fluorescence micrograph (i. e. manual image binning of the fluorescence raw data). (e) The x-ray dark field of this particular scan area now facilitates correlation of visible light fluorescence and $\mathrm{x}$-ray data, leading to a superimposition of datasets; Fig. 3.7 $(f)$ shows the x-ray dark field as a semitransparent top layer and the visible light fluorescence image as a non-transparent bottom layer. Translational shifts are estimated 'by eye' with an approximate accuracy of about one scanning step size (here: $\Delta=10 \mu \mathrm{m}$ ).

This correlation now allows the setting into relation of all observables determined on the fluorescence micrograph and the x-ray dataset: 

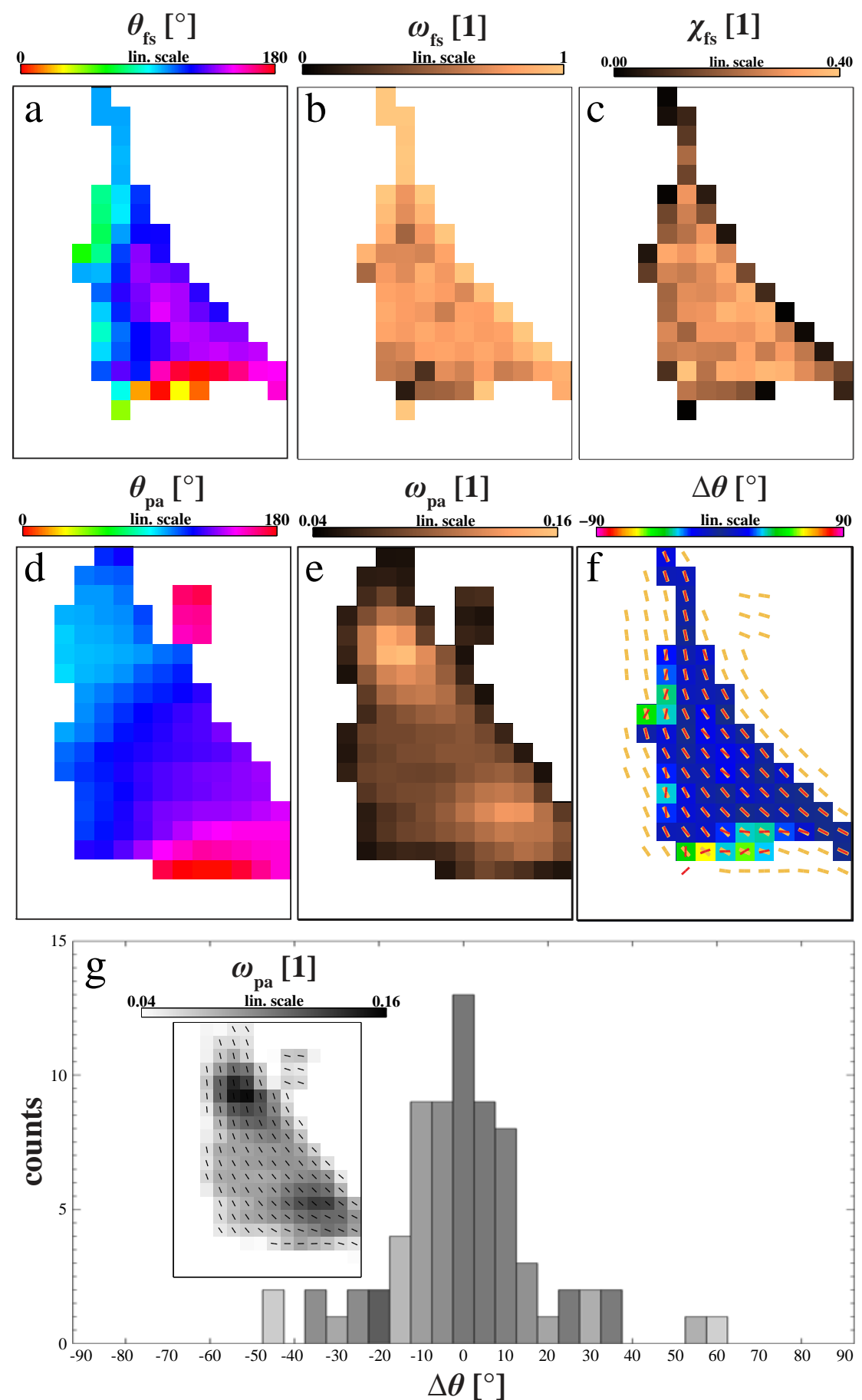

Figure 3.8: (a-c) Observables $\theta_{\mathrm{fs}}($ see $a), \omega_{\mathrm{fs}}($ see $b)$ and $\chi_{\mathrm{fs}}($ see $c)$ as found by the filament sensor [51). (d-e) Results for $\theta_{\mathrm{pa}}$ and $\omega_{\mathrm{pa}}$ as found by PCA on the x-ray dataset. (f) Direct comparison of the determined orientation angles $\theta_{\mathrm{fs}}$ (thin red lines) and $\theta_{\mathrm{pa}}$ (bold yellow lines). (g) Histogram of the angular deviation $\Delta \theta$, color-coded by the respective mean $\omega_{\mathrm{pa}}$-values. Figure and caption adapted from (43). 
Fig. $3.8(a-c)$ shows the three observables $\theta_{\mathrm{fs}}(a, b), \omega_{\mathrm{fs}}(a, b)$ and $\chi_{\mathrm{fs}}(a, b)$ on this particular scan area as found by the filament sensor; Fig. $3.8(d-e)$ shows the corresponding PCA results for $\theta_{\mathrm{pa}}(a, b)$ and $\omega_{\mathrm{pa}}(a, b)$ as determined from the x-ray data set, here thresholded to value of $\omega_{\mathrm{pa}}>0.05$.

Fig. $3.8(f): \theta_{\mathrm{fs}}(a, b)$ (thin red lines) and $\theta_{\mathrm{pa}}(a, b)$ (bold yellow lines) are directly comparable leading to an angular deviation

$$
\Delta \theta(a, b)=\theta_{\mathrm{fs}}(a, b)-\theta_{\mathrm{pa}}(a, b)
$$

that can be further exploited in a histogram, see $(g)$. The color code marks the mean $\omega_{\mathrm{pa}}$-value

$$
\Omega_{\text {bin }}=\frac{1}{N_{\text {bin }}} \sum_{i=1}^{N_{\text {bin }}} \omega_{\text {pa }}(a, b)
$$

for each bin. 


\subsection{Automated Analysis of Radial Intensity Profiles}

The analysis of local radial intensity profiles $I\left(q_{\mathrm{r}}\right)$ provides a detailed view on local form and structure factors of the scattering objects. However, when applying scanning SAXS, data grow to an extensive amount making it impossible to adequately map the characteristics of all local profiles of a scan in a meaningful manner. For example, broad $q_{\mathrm{r}}$-range data on the basis of the micro-SAXS recordings as detailed in Sec. 2.1 and Sec. 2.2 have led to local curves comprising about 1200 data points for each scan position. Together with a respective scan area up to $150 \times 150$ single diffraction spots for large area scans, an analysis remains elusive.

In order to reduce local profiles to a number of meaningful variables, a common approach is to describe them by a simple power law function following Eq. 2.5. However, more complex profiles as observed here and by others (e. g. source (29)) do not allow an adequate description of the signal prompting investigators to use two separate fitting approaches, one for low and one for high $q_{\mathrm{r}}$-values.

One ansatz to describe such signals with a single fitting function has already been introduced in Sec. 2.2.2 following Eq. 2.6. However, while an ansatz of this form is capable of describing the entire micro-SAXS data curve adequately well, it often takes several attempts to find suitable start parameters, sometimes leading to inconsistent fitting results so that a reliable description of an entire scan area is not possible.

A more robust approach can be derived from expanding Eq.2.5 with a linear combination of $q_{\mathrm{r}}^{p}$ with fixed integer exponents

$$
I_{\text {auto }}\left(q_{\mathrm{r}}\right)=\sum_{p=-\infty}^{\infty} c_{p} \cdot q_{\mathrm{r}}^{p}, p \in \mathbb{Z} .
$$

Further examination on a particular micro-SAXS dataset has revealed, that $I_{\text {auto }}\left(q_{\mathrm{r}}\right)$ can be restricted to an interval $p \in[-8,0]$ : Fig. $3.9(a-b)$ shows the x-ray dark fields of three single cells, see $(a)$, together with the intensity profiles of four different single diffraction spots, see $(b)$. 

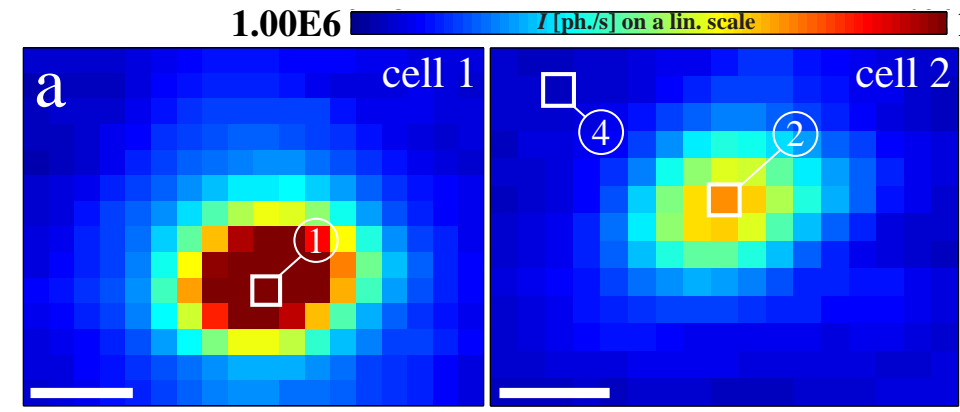

\subsection{E7}
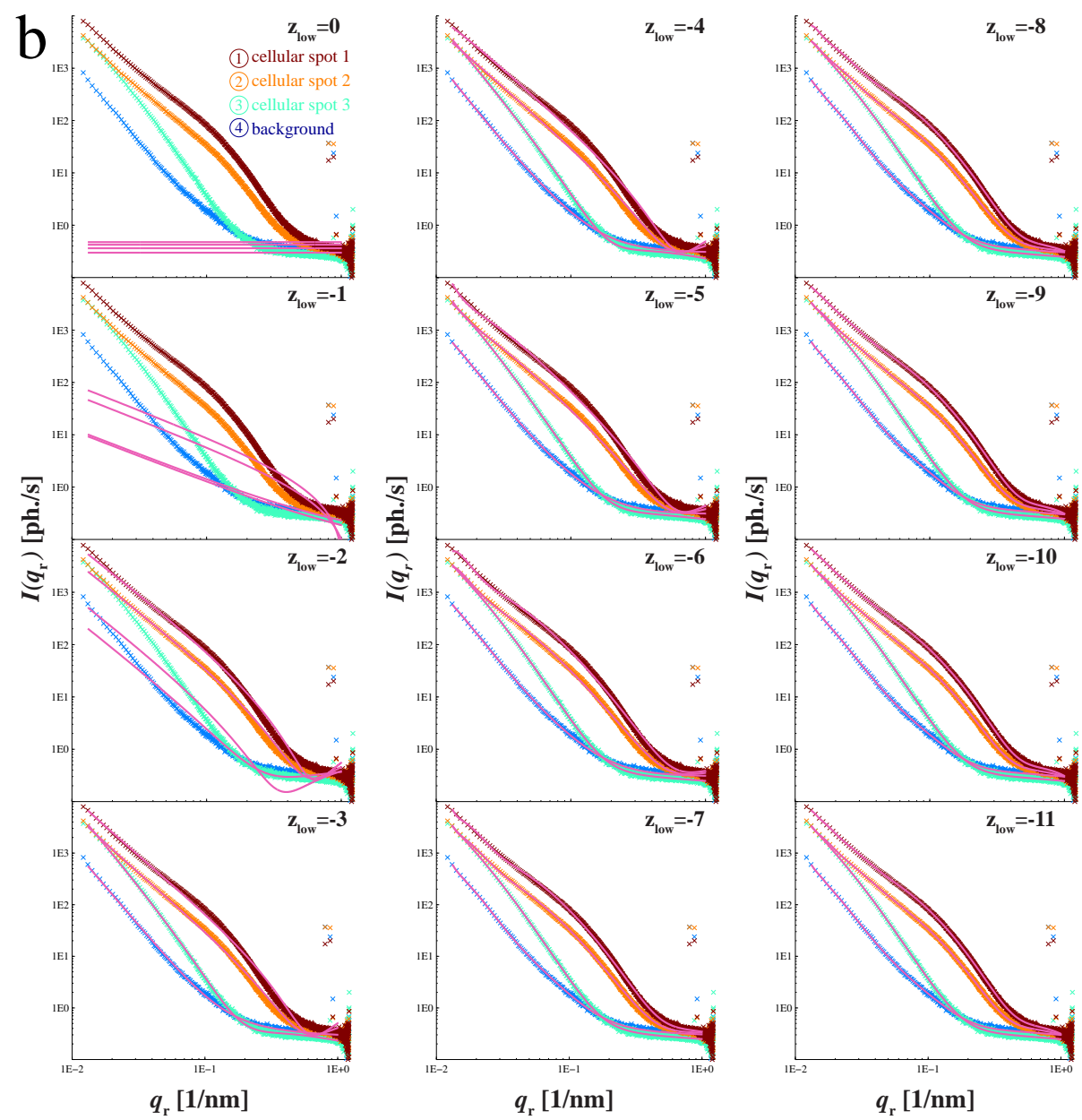

Figure 3.9: (a) X-ray dark fields of three NRCTCs recorded with the micro-SAXS setup. Scale bar: 40 $\mu$ m. (b) Radial intensity profiles $I\left(q_{\mathrm{r}}\right)$ of spots (1)-(4) as marked in (a). Profiles are described by $I_{\text {auto }}$ considering a linear combination of $c_{p} \cdot q_{\mathrm{r}}^{p}$, see Eq. $3.23 I_{\text {auto }}$ is restricted to integer exponents with fixed $z_{\text {high }}=0$ and a successively decreasing $z_{\mathrm{low}}=[-11,0]$ (up to down and left to right). Figure and caption adapted from 43 ). 
Pink lines indicate the development of the fitting function

$$
I_{\text {auto }}\left(q_{\mathrm{r}}\right)=\sum_{p=z_{\text {low }}}^{z_{\text {high }}} c_{p} \cdot q_{\mathrm{r}}^{p},
$$

with a fixed value for $z_{\text {high }}=0$ and a successively decreasing $z_{\text {low }} \rightarrow-11$. (1)-(3) originate from cellular diffraction signals with (1)-(2) revealing a kink in the profile while cellular spot (3) as well as background spot (4) show a constant power law decay. All intensity profiles can be described sufficiently well with $z_{\text {low }}=-8$, leading to nine observables $c_{-8}$ to $c_{0}$ for a micro-SAXS scan.

The coefficients $c_{p}$ are determined by a weighted least squares algorithm

$$
c=\left(V^{\mathrm{T}} \cdot W \cdot V\right)^{-1} \cdot\left(V^{\mathrm{T}} \cdot W \cdot I\left(q_{\mathrm{r}}\right)\right),
$$

with $V=\left(\begin{array}{lll}q_{\mathrm{r}}^{-8} & \ldots & q_{\mathrm{r}}^{0}\end{array}\right)$ (so-called design matrix), $W=\operatorname{diag}(w)$ with $w=\frac{1}{I\left(q_{\mathrm{r}}\right)^{1.5}}$ (weight matrix) and $I\left(q_{\mathrm{r}}\right)$ (response vector), see also (43, 53, 54) for more details.

Fig. 3.10 further illustrates the robustness of this procedure: Fig. 3.10 ( $a-b)$ shows the visible light micrograph of a freeze-dried NRCTC together with the x-ray dark field as obtained by scanning the sample with the micro-SAXS setup. (c) The local radial intensity profiles resulting from a (full) azimuthal integration of 2D-diffraction patterns can be reliably described in an automated manner by $I_{\text {auto }}\left(q_{\mathrm{r}}\right)$ (pink lines), with limits $z_{\text {low }}=-8$ and $z_{\text {high }}=0$ following Eq. 3.23

From this computation, further (possibly angle-dependent) variables can be derived, such as the zeroth (i. e. the intensity) and second momentum $F_{0}\left(q_{\mathrm{r}}\right) \& F_{2}\left(q_{\mathrm{r}}\right)$ with

$$
\begin{gathered}
F_{0}\left(q_{\mathrm{r}}\right)=\int_{q_{\min }}^{q_{\max }} I_{\text {auto }}\left(q_{\mathrm{r}}\right) \mathrm{d} q_{\mathrm{r}}=c_{0}+\left.c_{-1} \cdot \ln \left(q_{\mathrm{r}}\right)\right|_{q_{\mathrm{r}}=q_{\min }} ^{q_{\max }}+\left.\sum_{i=-8}^{-2} \frac{1}{i+1} c_{i} \cdot q_{\mathrm{r}}^{i+1}\right|_{q_{\mathrm{r}}=q_{\min }} ^{q_{\max }}, \\
F_{2}\left(q_{\mathrm{r}}\right)=\int_{q_{\min }}^{q_{\max }} q_{\mathrm{r}}^{2} \cdot I_{\mathrm{auto}}\left(q_{\mathrm{r}}\right) \mathrm{d} q_{\mathrm{r}}=\int_{q_{\min }}^{q_{\max }} \sum_{p=-8}^{0} c_{p} \cdot q_{\mathrm{r}}^{p+2} \mathrm{~d} q_{\mathrm{r}} \\
=\left.c_{-3} \cdot \ln \left(q_{\mathrm{r}}\right)\right|_{q_{\mathrm{r}}=q_{\min }} ^{q_{\max }}+\sum_{i=-8}^{-4} \frac{1}{i+3} \cdot \frac{c_{i}}{q_{\mathrm{r}}^{i+3}}+\left.\sum_{i=-2}^{0} \frac{1}{i+3} \cdot \frac{c_{i}}{q_{\mathrm{r}}^{i+3}}\right|_{q_{\mathrm{r}}=q_{\min }} ^{q_{\max }},
\end{gathered}
$$

as well as the quadratic displacement $<q_{\mathrm{r}}^{2}>=F_{2}\left(q_{\mathrm{r}}\right) / F_{0}\left(q_{\mathrm{r}}\right)$ and the experimental limits $I_{\text {auto }}\left(q_{\min }\right)$ and $I_{\text {auto }}\left(q_{\max }\right)$ of structures that can be resolved 43). 

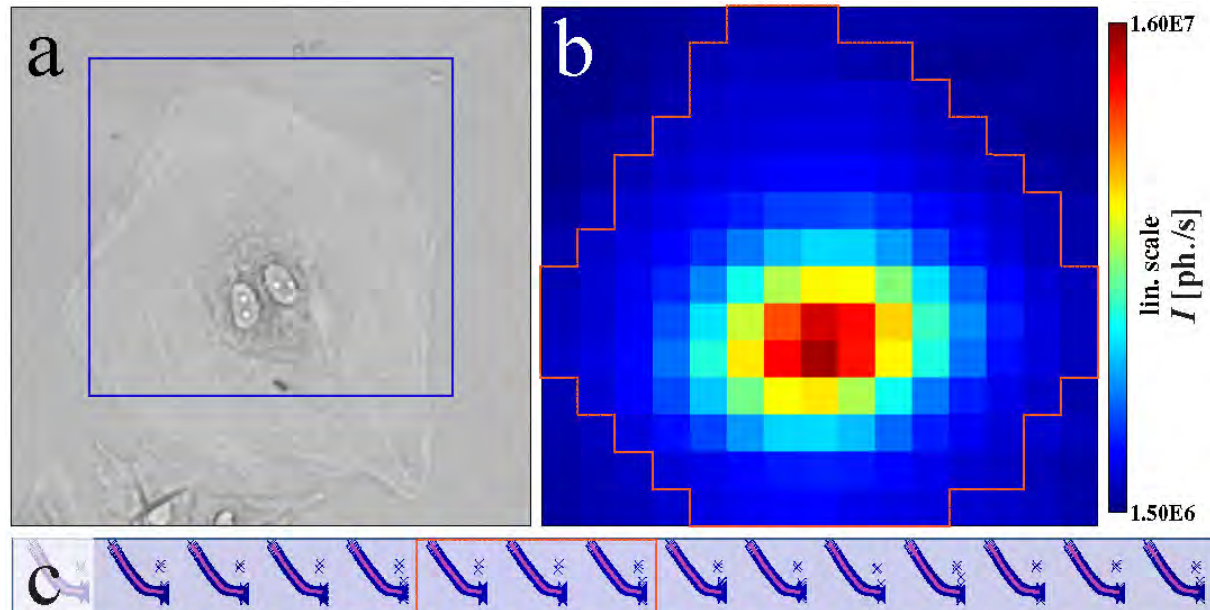
$L_{*}^{*} L_{*}^{*} L_{*}^{*} L_{*}^{*} L_{*}^{*} L_{*}^{*} L_{*}^{*} L_{*}^{*} L_{*}^{*} L_{*}^{*} L_{*}^{*} L_{*}^{*} L_{*}^{*}$ $L_{*}^{*} L_{*}^{*} L_{*}^{*} L_{*}^{*} L_{*}^{*} L_{*}^{*} L_{*}^{*} L_{*}^{*} L_{*}^{*}$

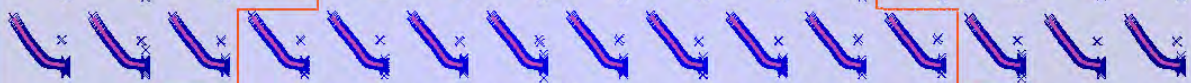
$L_{*}^{*} L_{*}^{*} L_{*}^{*} L_{*}^{*} L_{*}^{*} L_{*}^{*} L_{*}^{*} L_{*}^{*} L_{*}^{*} L_{*}^{*} L_{*}^{*}$ $L_{*}^{*} L_{*}^{*} L_{*}^{*} L_{*}^{*} L_{*}^{*} L_{*}^{*} L_{*}^{*} L_{*}^{*} L_{*}^{*} L_{*}^{*}$ $L_{*}^{*} L_{*}^{*} L_{*}^{*} L_{*}^{*} * L_{*}^{*}$

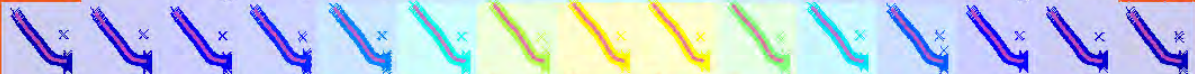
$L_{*}^{*} L_{*}^{*} L_{*}^{*} * v_{*}^{*} v_{*}^{*}$ $\left.L_{*}^{*} L_{*}^{*} L_{*}^{*} L_{*}^{*}\right|_{*} ^{*} V_{*}^{*}$ $L_{*}^{*} L_{*}^{*} L_{x}^{*} * V_{*}^{*}$

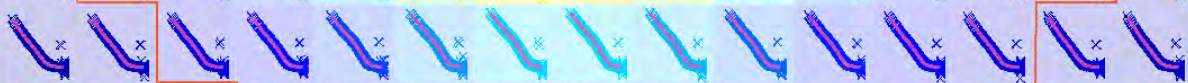

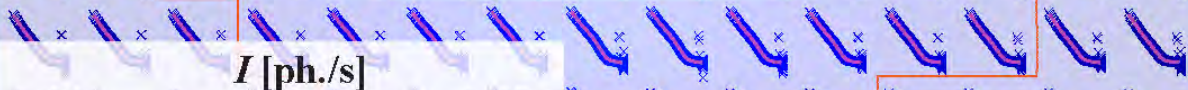
$1.50 \mathrm{E} 6 \quad$ lin. scale $1.60 \mathrm{E} 7 \mathrm{~N}_{*}^{*} v_{*}^{*}$

Figure 3.10: (a) Visible light bright field micrograph of a NRCTC. (b) X-ray dark field of the respective area. (c) Local intensity profiles for all scan points including the overall scattering intensity as color code and the respective robust fit results as pink lines. Figure and caption adapted from 43. 


\section{Section 4}

\section{A SAXS Study of Stem Cells}

\section{This section includes excerpts of (42).}

The combination of a scanning SAXS setup for biological cells as introduced in Sec. 2.1 2.2 together with the automated analysis tools as described above enables detailed and highly resolved structural studies of various eukaryotic cell lines. This section focuses on scanning SAXS experiments performed on human mesenchymal stem cells (hMSCs) and differentiated cell lines addressing issues of preparation, data acquisition and optics, analysis and finally results:

Sec. 4.1 presents first micro-SAXS results indicating structural differences between naive and muscle-induced hMSCs, murine myo- and fibroblasts. These observations are further analyzed in a statistical manner in Sec. 4.2 based on four structural observables quantifying the average scattering behavior of single cells. Towards a higher resolution in real space, Sec. 4.34 .6 presents results from nano-SAXS scans introducing high signal data obtained from freeze-dried samples in Sec. 4.34 .4 also addressing cryoprotection, which can minimize radiation damage, before turning to alternative sample preparation methods including first results on frozen-hydrated and hydrated cells in Sec. 4.5 4.6 . 


\subsection{Micro-SAXS on Freeze-Dried Cells}

Scanning micro-SAXS as implemented at cSAXS-endstation of the Swiss Light Source permits the scanning of large areas in a relatively small amount of time, probing all structures present at the illumination spot as a broad local average of about $30 \times 50 \mu \mathrm{m}$ on the sample plane. The corresponding $2 \mathrm{D}$ diffraction patterns provide an extraordinarily clean diffraction signal without disturbing empty-beam features. These attributes make possible the rapid probing of a large number of cells (as will be taken advantage of in Sec. 5.2 resulting in arrays of diffraction patterns with a broad $q$-range signal, that can be further exploited by automated algorithms detailed in Sec. 2.2.1 Sec. 3.1 and Sec. 3.3

In order to test if scanning SAXS is capable of identifying the structural details of cells, several scans are performed on freeze-dried stem cells and differentiated cell lines: Fig. 4.1 shows a micro-SAXS scan of a freeze-dried muscle-induced hMSC including the OAV-micrograph in $(a)$ and the x-ray dark field in $(b)$. (c) PCA-results (restricted to the area framed in $(b)$, pink line) indicate a rather inhomogeneous distribution of anisotropies as quantified by $\omega_{\text {pa }}$ (color-code). The corresponding structure orientation angles $\theta_{\mathrm{pa}}$ (white lines) are correlated among neighboring pixels, but reveal a significant variation throughout the entire cell area. A preferred structure orientation is indicated by the so-called 'director' of the set (black arrow) as will be further detailed in Sec. 4.2 Fig. 4.1 (d) shows the composite image of diffraction patterns including the two rescaled eigenvectors $\vec{l}_{k}(a, b)$ (see Eq. 3.8) as green and red arrows.

Comparing these results to selected scans of other cell lines leads to first indications of differences in their scattering behavior: Fig. 4.2 shows corresponding OAV-images, $\mathrm{x}$-ray dark fields and the PCA results (restricted to cellular areas; see respective dark field maps, pink frame) of a naive hMSC in $(a-c)$, a murine myoblast in $(d-f)$ and a murine fibroblast in ( $g-i)$. Together with Fig. 4.1. data reveal x-ray dark field intensities that differ by more than two orders. PCA results further show a high consistency of structure orientation angles in differentiated murine cell lines, and significant variation in $\theta_{\mathrm{pa}}$ for both hMSC-derived examples. This becomes clear when plotting structure orientation angles $\theta_{\text {pa }}$ of all four datasets in a histogram, see Fig. 4.3 naive and muscle-induced hMSCs lead to a relatively broad spectrum of orientation angles, while the evaluations of murine cell lines present a rather sharp distribution around one dominating main orientation.

With further scan data available (see also Appendix Sec. 10.3, these observations will be discussed in a statistical manner in the following Sec. 4.2 

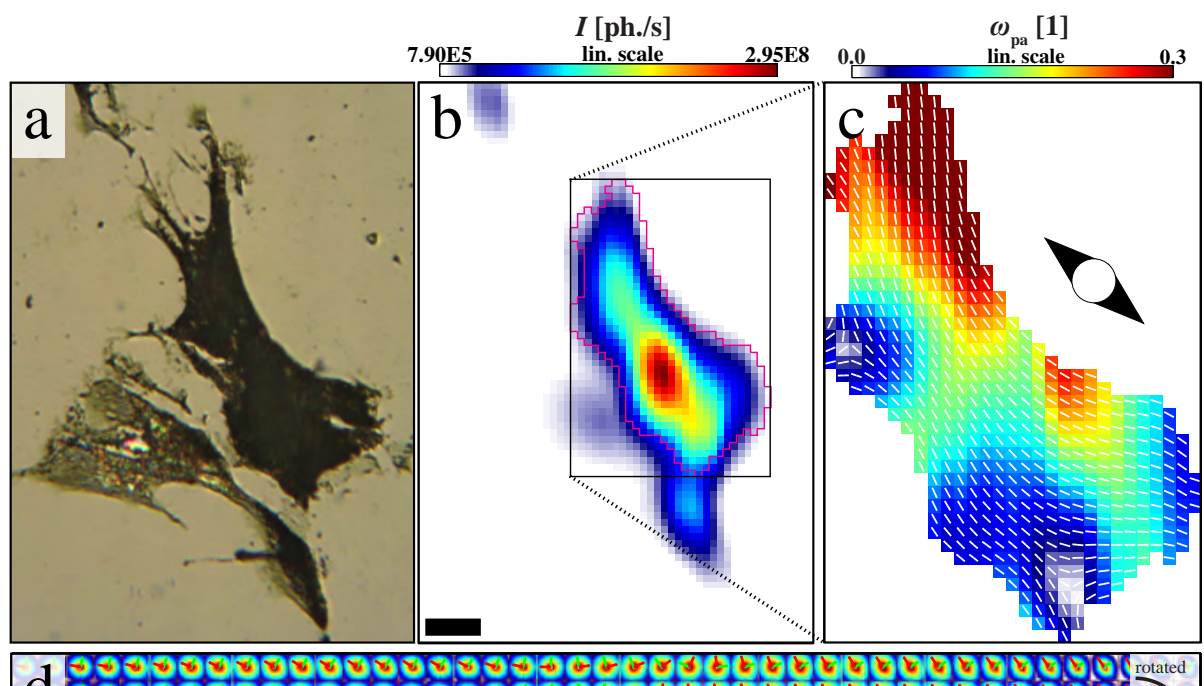

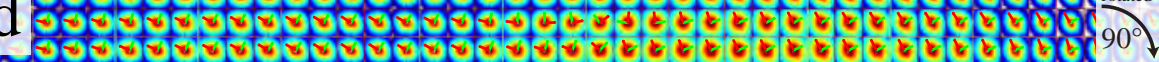

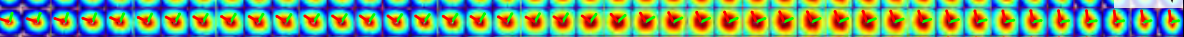

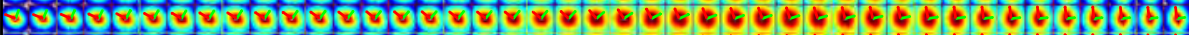

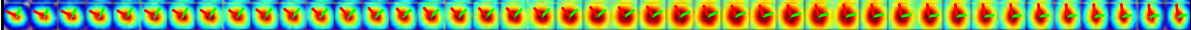

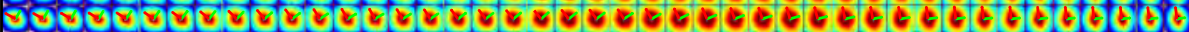

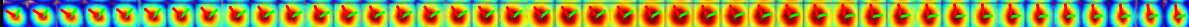

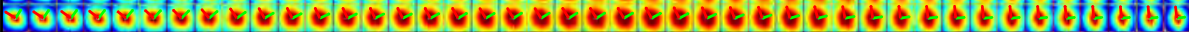

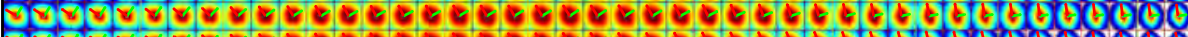

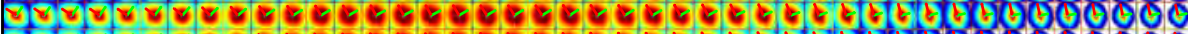

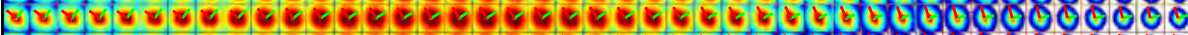

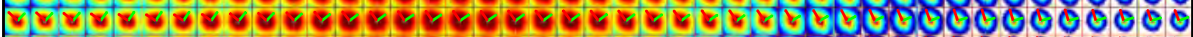

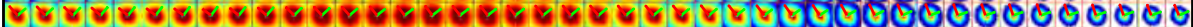

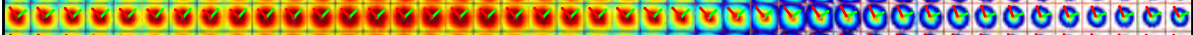

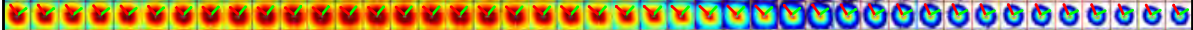

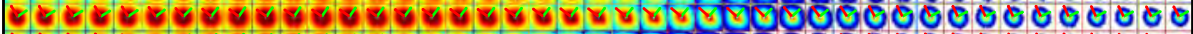

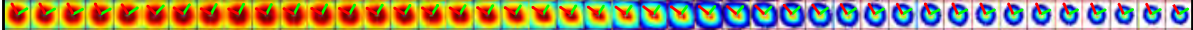

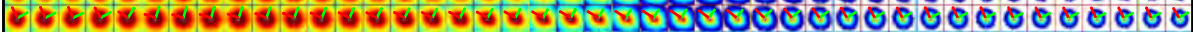

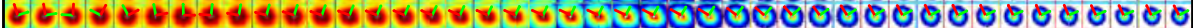

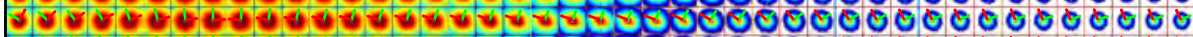

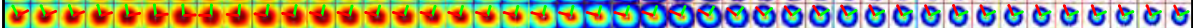

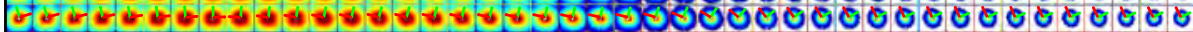
निम

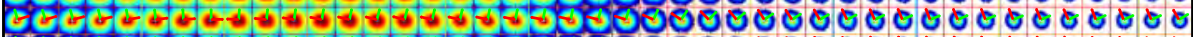

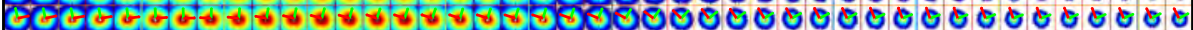

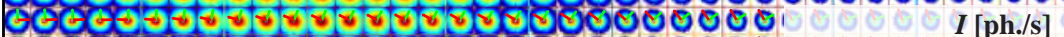

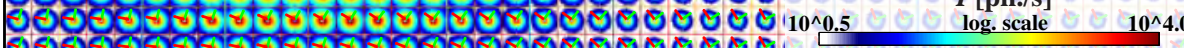

Figure 4.1: (a) OAV micrograph of a freeze-dried mi-hMSC right before recording. (b) X-ray dark field as obtained by a micro-SAXS scan with a step size of $\Delta=5 \mu \mathrm{m}$. Scale bar: $40 \mu \mathrm{m}$. (c) PCA results of the region marked in (b, pink frame) showing the structure orientation angles $\theta_{\mathrm{pa}}$ as white lines and the order parameter $\omega_{\mathrm{pa}}$ as color code. All contributions outside this mask are set to zero. (d) Composite image of the scan area (rotated clockwise by $90^{\circ}$ ) including the two rescaled eigenvectors $\vec{l}_{k}(a, b), k \in[1,2]$, as green and red arrows. Figure and caption adapted from [42]. 

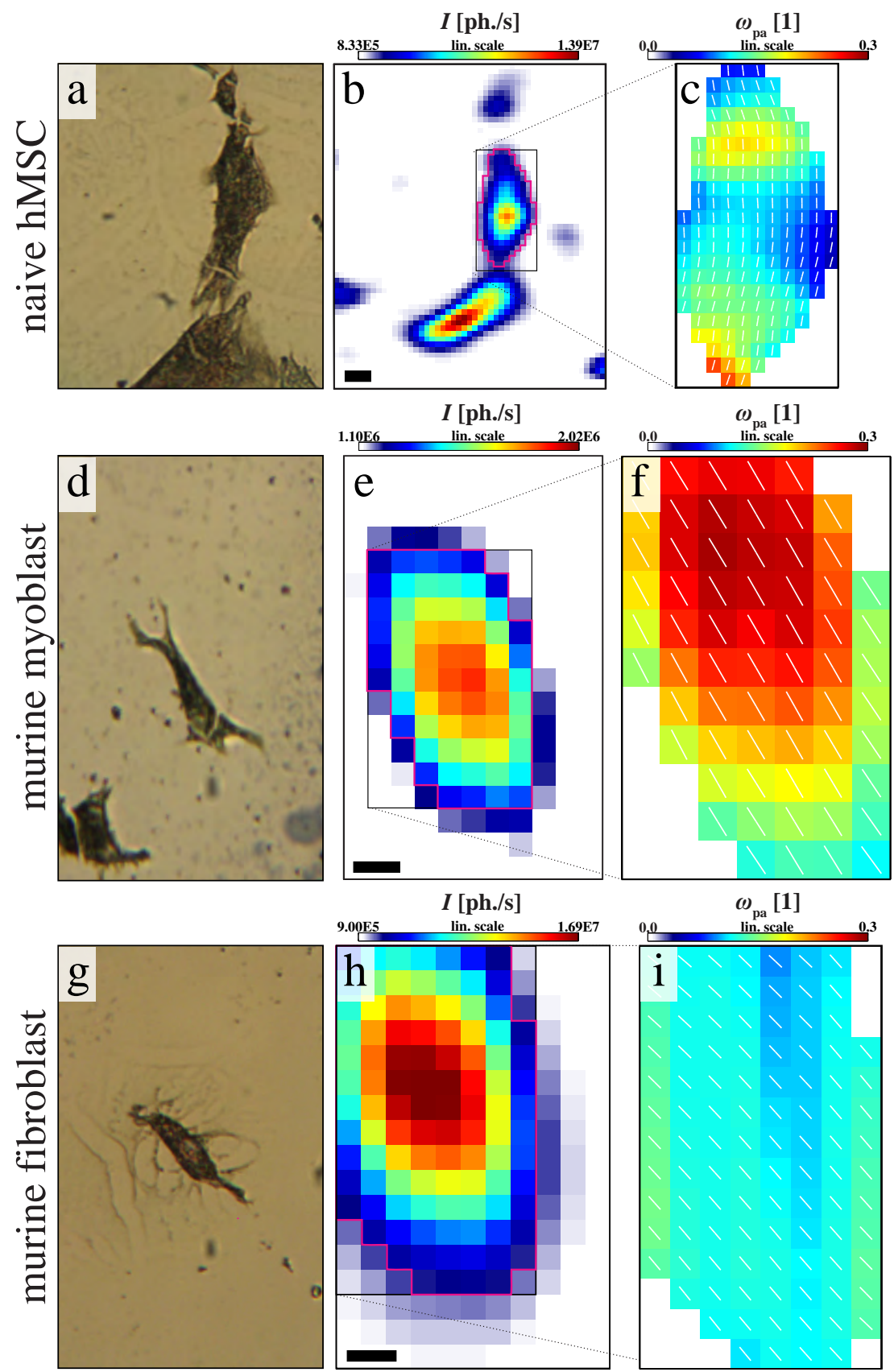

Figure 4.2: (a-i): OAV-micrograph, $x$-ray dark field and PCA results for a naive hMSC (a-c), a murine myoblast (d-f) and a murine fibroblast (g-i) as obtained by micro-SAXS scanning with step sizes of $\Delta=8$, 5 and $5 \mu \mathrm{m}$, following the same procedure as described for Fig.4.1 Scalebars: 40, 10 and $10 \mu \mathrm{m}$. Figure and caption adapted from [42]. 

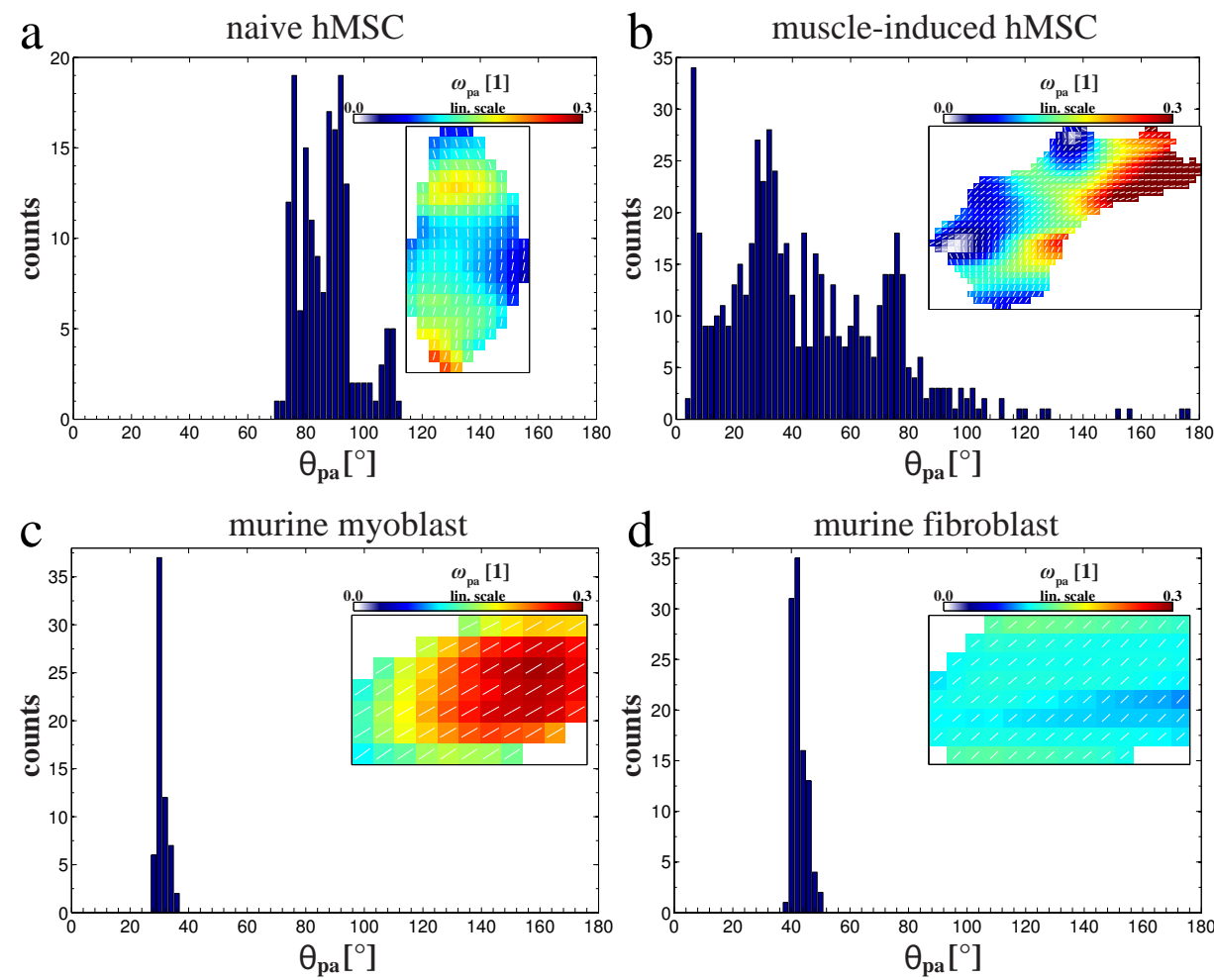

Figure 4.3: $(a-d)$ Histograms showing the distribution of structure orientation angles $\theta_{\mathrm{pa}}$ as determined by PCA for a naive and a muscle-induced hMSC, see ( $a \& b)$, as well as a murine myoblast and $a$ murine fibroblast, see ( $c \& d$ ). Figure and caption adapted from 42). 


\subsection{Micro-SAXS Analysis: Statistics \& Definition of Cellu- lar Diffraction Observables}

Automated algorithms such as the dark field analysis tool (see Sec. 2.2.1, the principal component analysis (see Sec. 3.1 or the automated robust fitting algorithm (see Sec.3.3 provide the means for the examination of particular aspects of a scan, which is valuable for a single dataset. However, in order to obtain a statistically reliable statement for the behavior of a biological species, it is necessary to condense the information into a few meaningful observables, each reflecting the different aspects of interest. The present section gives an example in this respect by introducing four scanning SAXS-derived observables, considering

- the mean scattering intensity $\langle I\rangle$ as a measure for the overall scattering strength,

- the mean order parameter $\Omega$ as a measure for the overall anisotropic character,

- the 2D nematic order parameter $s$ as a measure for orientational correspondence as well as

- the mean standard deviation of the momentum transfer $\langle\bar{\sigma}\rangle$

of a cell.

$\langle I\rangle$ is obtained directly from the x-ray dark field

$$
\langle I\rangle=\frac{1}{N_{\mathrm{ROI}}} \sum_{a,\left.b\right|_{\mathrm{ROI}}} I_{\mathrm{df}}(a, b)
$$

with the dark field intensity $I_{\mathrm{df}}(a, b)$ (as defined by Eq. 2.1) at scan position $(a, b) \in \mathbb{N}^{2}$ considering all diffraction patterns within a ROI-mask. All masks are defined based on the x-ray dark field extracting separable single cells. Accordingly, $N_{\mathrm{ROI}}$ denote the total number of scan positions within the ROI-mask. $\Omega$ is derived from the mean value of all local $\omega_{\mathrm{pa}}(a, b)$ within the single cell region

$$
\Omega=\frac{1}{N_{\mathrm{ROI}}} \sum_{a,\left.b\right|_{\mathrm{ROI}}} \omega_{\mathrm{pa}}(a, b) .
$$


The nematic order parameter $s$ can be calculated from the structure orientation angle $\theta_{\mathrm{pa}}(a, b)$

$$
s=\frac{1}{N_{\mathrm{ROI}}} \sqrt{\left(\sum_{a,\left.b\right|_{\mathrm{ROI}}} \sin \left(2 \theta_{\mathrm{pa}}(a, b)\right)\right)^{2}+\left(\sum_{a,\left.b\right|_{\mathrm{ROI}}} \cos \left(2 \theta_{\mathrm{pa}}(a, b)\right)\right)^{2}},
$$

(with further intermediate steps shown in (42)). This result can be derived from the second rank tensor $Q$ following the work of Liu et al. (55):

$$
Q_{\alpha \beta}=\frac{1}{N_{\mathrm{ROI}}} \sum_{a,\left.b\right|_{\mathrm{ROI}}}\left(2 e_{\mathrm{pa}, \alpha}(a, b) e_{\mathrm{pa}, \beta}(a, b)-\delta_{\alpha \beta}\right)
$$

with $\alpha, \beta \in[1,2]$ and the unit vector

$$
\vec{e}_{\mathrm{pa}}(a, b)=\left(\begin{array}{l}
e_{1}(a, b) \\
e_{2}(a, b)
\end{array}\right)=\left(\begin{array}{l}
\cos \left(\theta_{\mathrm{pa}}(a, b)\right) \\
\sin \left(\theta_{\mathrm{pa}}(a, b)\right)
\end{array}\right) .
$$

that is directed along the principal axis. Eq. 4.4 can thus be written as

$$
Q=\frac{2}{N_{\mathrm{ROI}}} \cdot\left(\begin{array}{cc}
\sum_{a,\left.b\right|_{\mathrm{ROI}}}\left(\left(\cos \left(\theta_{\mathrm{pa}}(a, b)\right)\right)^{2}-\frac{1}{2}\right) & \sum_{a,\left.b\right|_{\mathrm{ROI}}} \cos \left(\theta_{\mathrm{pa}}(a, b)\right) \sin \left(\theta_{\mathrm{pa}}(a, b)\right) \\
\sum_{a,\left.b\right|_{\mathrm{ROI}}} \sin \left(\theta_{\mathrm{pa}}(a, b)\right) \cos \left(\theta_{\mathrm{pa}}(a, b)\right) & \sum_{a,\left.b\right|_{\mathrm{ROI}}}\left(\left(\sin \left(\theta_{\mathrm{pa}}(a, b)\right)\right)^{2}-\frac{1}{2}\right)
\end{array}\right) .
$$

The eigenvalues $s_{k}, k \in[1,2]$, of the equation

$$
Q \cdot \overrightarrow{\tilde{u}}=s \cdot \overrightarrow{\tilde{u}}
$$

then lead to the 2D nematic order parameter $s$, corresponding to the positive eigenvalue $s_{k}>0$. The eigenvector $\overrightarrow{\tilde{u}}_{s}$ is the director of the ROI, reflecting the preferred orientation of diffraction patterns within the area.

$\langle\bar{\sigma}\rangle$ considers the average of variances $\lambda_{1}$ and $\lambda_{2}$ at each scan point within the area,

$$
\langle\bar{\sigma}\rangle=\frac{\sum_{a,\left.b\right|_{\mathrm{ROI}}} \bar{\sigma}(a, b)}{N_{\mathrm{ROI}}}=\frac{\sum_{a,\left.b\right|_{\mathrm{ROI}}} \sqrt{\frac{\lambda_{1}(a, b)+\lambda_{2}(a, b)}{2}}}{N_{\mathrm{ROI}}},
$$

thus referring to the mean scattering width of the signal.

Fig 4.4 shows the results for $\langle I\rangle, \Omega, s$ and $\langle\bar{\sigma}\rangle$ on the basis of multiple micro-SAXS scans performed on freeze-dried naive and muscle-induced hMSCs (mi-hMSC), murine myo- (C2C12) and fibroblasts (NIH-3T3), see Appendix Sec. 10.3 for further details. All 
cells are grown on $1 \mu \mathrm{m}$-thick silicon nitride windows, cryofixated in the alive state and then freeze-dried. Scans are performed under equivalent conditions (photon energy $E_{p h}=8.7 \mathrm{keV}$, photon flux $I_{0}=1.44 \cdot 10^{11} \mathrm{ph} . / \mathrm{s}$, dwell time $t_{\text {dwell }}=0.5 \mathrm{~s}$, no cryoprotection) applying scanning step sizes of $\Delta=5-10 \mu \mathrm{m}$. The respective mean $\mu=1 / N \cdot \sum_{i} y_{i}$ value of observable $y_{i}$ for each cell is depicted as bars, together with the standard deviation $S=\left(1 /(N-1) \cdot \sum_{i}\left|y_{i}-\mu\right|^{2}\right)^{1 / 2}$ depicted as error bars.
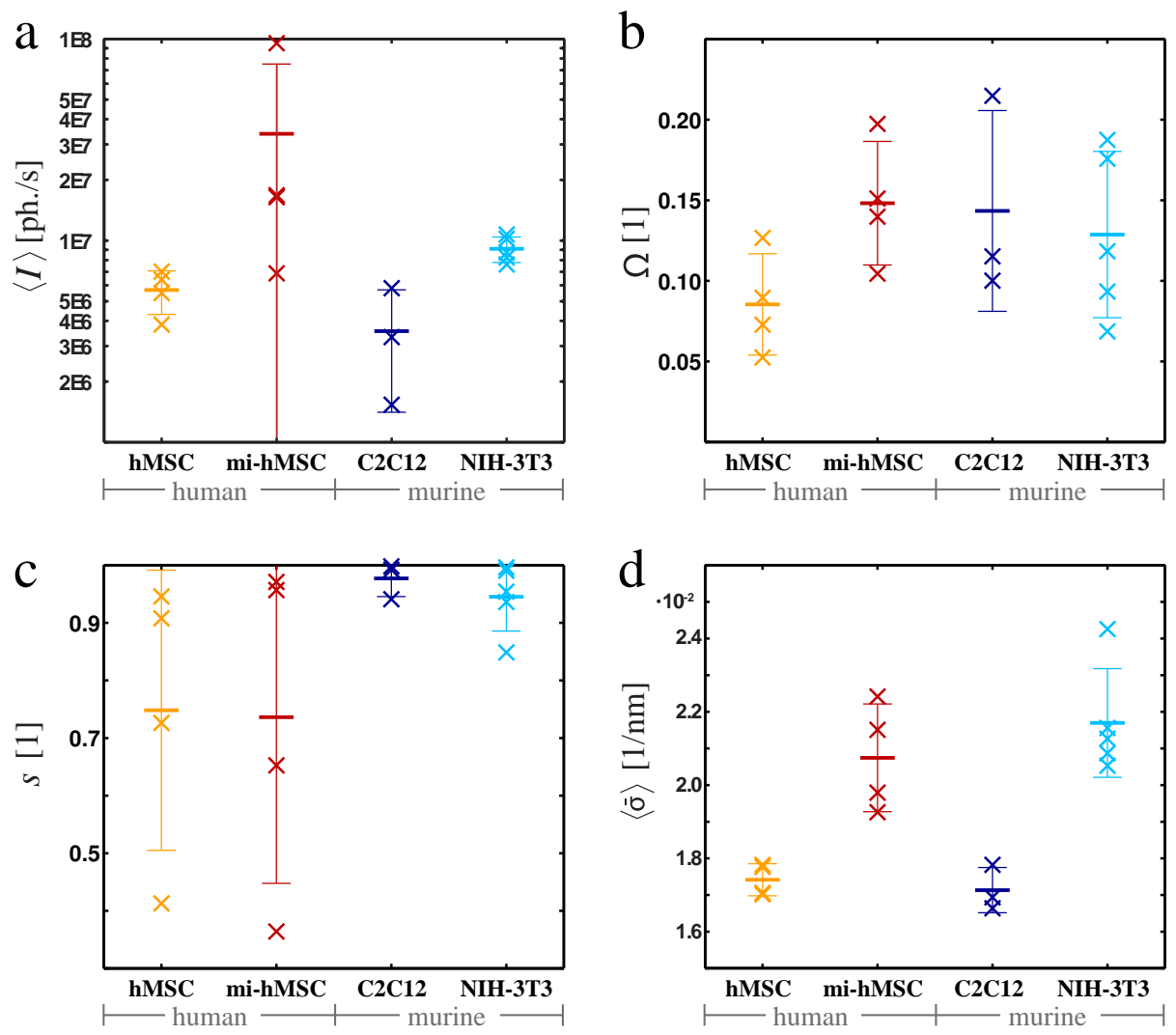

Figure 4.4: Statistical analysis of different freeze-dried cell lines considering (a) the mean scattering intensity $\langle I\rangle$, (b) the mean order parameter $\Omega$, (c) the $2 D$ nematic order parameter s and (d) the mean standard deviation of the momentum transfer $\langle\bar{\sigma}\rangle$. Figure and caption adapted from [2].

Fig. 4.4 (a): The mean scattering intensity $\langle I\rangle$ shows noticeable differences among all cell lines tested, revealing an increase from naive to muscle-induced hMCSs and from murine myo- to fibroblasts. Fig. 4.4 (b) shows all respective values for $\Omega$ revealing a shift between naive hMSCs and all other cell types, indicative for naive stem cells to 
be rather unstructured. $(c)$ The statistical values of $s$ obtained for naive and muscleinduced hMSCs exhibit a relatively large diversity between orientation angles, which is in line with first observations made in Sec. 4.1. (d) Relatively large values $\langle\bar{\sigma}\rangle$ for mi-hMSCs and murine fibroblasts imply scattering towards high $q$-values that, along with the observations made for $\Omega$, are caused by small, ordered structures within the cells.

Of course, addressing the differentiation process of stem cells will require more efforts regarding the quantity of scanned cells as well as sample preparation: Ideally, a quantitative SAXS study should exclusively rely on stem cells in the naive and (induced) committed or differentiated state, all originating from the same cellular source; the respective cell fate can be predefined and controlled throughout the incubation process by well-known specific chemical and physical parameters $(2,3,10,56,57)$. A large quantity of scans further ensures reliable statistics, keeping in mind that comparative studies based on more established imaging techniques rely on quantities of 60 cells or more for each condition (see e.g. (10)). The data shown here lead to large error bars which indicate unreliable statistics. Notwithstanding, results shown already illustrate the potential of scanning SAXS, that can possibly be further evolved into a powerful tool thus complementing observations in stem cell research. If feasible, scanning with a significantly enhanced real space resolution may provide access to the structural properties of particular organelles, which can then be further characterized in a statistical manner by observables as the ones defined above.

\subsection{Increasing The Real-Space Resolution by Nano-SAXS}

As a first step towards increasing the real-space resolution, the following sections (Sec. 4.3-4.6 present multiple nano-SAXS results of samples in different preparation states addressing methodological and biophysical topics. All nano-SAXS experiments shown are performed with a beam size of a few hundred nanometers in the focal spot (see Sec. $2.1 \& 2.2$ for details) and with a scanning step size of typically $\Delta=0.5-1 \mu \mathrm{m}$.

Fig. 4.5 illustrates the imaging capabilities of state of the art nano-SAXS instruments by showing a representative dataset of a freeze-dried mi-hMSC on an ultrathin silicon nitride window. Fig. 4.5 (a) shows the $40 \times$ visible light phase contrast micrograph of the cell before cryoplunging. The approximated scan area is framed in red. (b) The $\mathrm{x}$ ray dark field reveals an overall scattering intensity that nicely outlines the morphology of the cell. A change in scattering strength allows to distinguish the nucleus from the 
surrounding cytoplasm. Anisotropies are identified by applying the streak finder algorithm (briefly introduced in Sec. 3.1 detailed in (31)) to the dataset: (c) The corresponding (anisotropy) orientation angles (black lines) are thresholded to a value of $\breve{S}>0.09$. Results indicate large areas of uniform orientation, that are predominantly found on the cortex, indicative for filamentous structures going alongside the cell's perimeter. Fig. $4.5(d)$ exemplarily shows a composite image of diffraction patterns located at the position marked in $(c)$. In agreement with previous observations, data reveal rather pronounced anisotropic scattering profiles, here shown for a small excerpt of the detector around the primary beam position. The use of semitransparent beam stops (indicated by rectangular shadows) allow a further analysis of diffraction patterns including the primary beam signal, see Sec.2.2.1 for details.

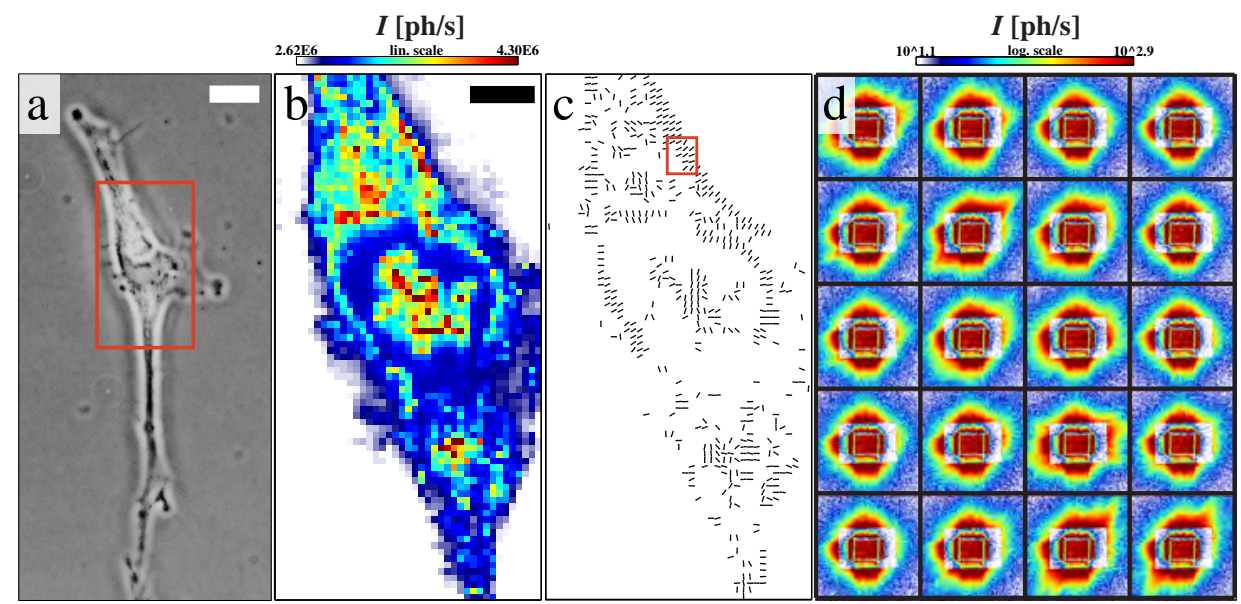

Figure 4.5: (a) 40× visible light phase contrast image of a mi-hMSC on a silicon nitride window before plunging of the sample. Scale bar: $20 \mu \mathrm{m}$. (b) X-ray dark field of a nano-SAXS scan with an approximated scan area as marked in (a). Scale bar: $5 \mu \mathrm{m}$. (c) Streak finder results on the x-ray dataset showing the anisotropy orientation angles as black lines (d) $2 D$ diffraction patterns of the region marked in (c) revealing pronounced anisotropic signals on the perimeter of the cell. Figure and caption adapted from [42).

\subsection{Nano-SAXS from Cryoprotected Cells}

The high flux of photons generated from a synchrotron source, in this work typically around $10^{11} \mathrm{ph} . / \mathrm{s}$, inevitably causes structural damage in the sample, which for cells can manifest itself in a significant steepening of the radial intensity decay and a loss in scattering intensity at each scan point (31). In particular nano-SAXS instruments, 
which restrict the photon flux to a small spot, limit the experiment to basically one finely rastered scan for each sample region $[27,31,58)$. The damage can be monitored by (low-dose) x-ray holograms before and after the SAXS scan (59).

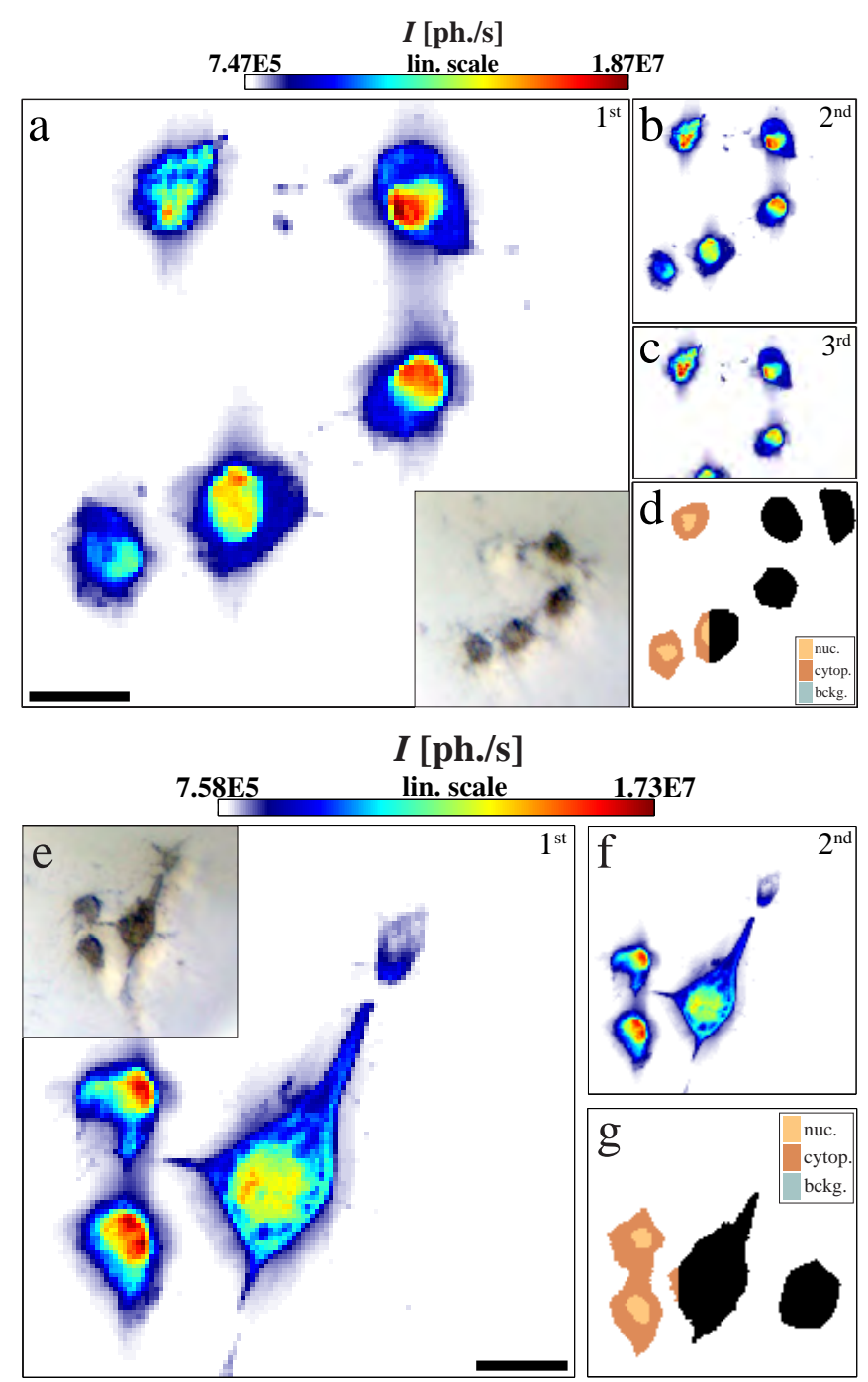

Figure 4.6: (a-c) X-ray dark field maps of freeze-dried murine fibroblasts ("5-cell arrangement") on ultrathin silicon nitride windows probed with a nano-SAXS beam while the sample is kept under cryogenic conditions. (d) ROI-mask defining nucleic and cytoplasmic regions for further quantification. (eg) X-ray dark field maps and ROI-mask of freeze-dried murine fibroblasts ("3-cell arrangement") with the same specifications and illustration outlined in $(a-d)$. Figure and caption adapted from (42). 
One approach to minimize structural alterations in freeze-dried samples is to apply cryogenic protection to the specimen, enabling proteins and nucleic acids to "withstand doses at least two orders of magnitude larger than room temperature samples" (58). Addressing this topic, a scan series of three or respectively two consecutive scans have been performed on freeze-dried murine fibroblasts, while the sample is kept under the cryogenic jet of a Cryostream (Oxford Instruments, UK).

Fig. 4.6 shows the results for two scan series comprising the x-ray dark fields of a "5-cell arrangement" that is successively scanned three times $(a-c)$ as well as of a "3cell arrangement" that is scanned two times $(e-f)$. Insets of $(a)$ and $(e)$ show the OAVmicrographs of the scan area right before recording.

The theoretical radiation dose applied on the sample can be determined by

$$
D=\frac{I_{0} \cdot \tau \cdot E_{\mathrm{ph} .}}{l_{\mathrm{att}} \cdot \rho \cdot \Delta_{y} \cdot \Delta_{z}}
$$

with photon flux $I_{0}[\mathrm{ph} . / \mathrm{s}]$, dwell time $\tau$ [s], photon energy $E_{\mathrm{ph}}=7.9 \mathrm{keV}$, beam dimensions $\Delta_{y}$ and $\Delta_{z}$ (FWHM) and attenuation length $l_{\text {att }}=7.2 \cdot 10^{-4} \mathrm{~m}$ at the given photon energy as obtained for a hypothetical molecule $\mathrm{H}_{50} \mathrm{C}_{30} \mathrm{~N}_{9} \mathrm{O}_{10} \mathrm{~S}$ with a density $\rho=1.35 \mathrm{~g} / \mathrm{cm}^{3}$ modelling the average composition of a biological cell (27, 60). Inserting the scan parameters of the datasets shown in Fig. 4.6 leads to a theoretical dose of $D \approx 2.1 \cdot 10^{8}$ Gy applied by each scan.

So far, both scan series reveal no significant degradations in the intensity footprint of x-ray dark field maps. In order to further quantify potential structural alterations, average radial intensity profiles are computed for each scan of the series. To this end, ROI-masks are defined based on the respective first x-ray dark field of each scan series separating nucleic, cytoplasmic and background regions, see $(d)$ and $(g)$. These masks are then adapted to all following scans respecting lateral shifts and differing step sizes (not shown), which further provides the means to calculate 2D average diffraction patterns for each particular area and each scan of the series. The corresponding radial intensity profiles $I\left(q_{\mathrm{r}}\right)$ are obtained by (full) azimuthal averaging of these signals. Fig. 4.7 shows all (background-corrected) data including the fitting curves (red lines) as obtained by a simple power law function (see Eq.2.5; Results are listed in Tab.4.1. No significant steepening of radial intensity profiles were observed: results reveal only minor alterations of the exponents $b$ within a scan series.

In conclusion, results show that cryogenic protection preserves freeze-dried cells sufficiently well during scanning SAXS experiments, thus enabling the combination of multiple high-dose x-ray methods such as nano-SAXS, ptychography and (with an appropriate setup) tomography on the same scan area. 
From a practical point of view, the mounting procedure is very simple requiring (i) a mechanical fixation of the sample, (ii) the approach of the (running) cryo-nozzle in one straight movement and then (iii) the alignment of the sample parallel to the gas stream to avoid any ice crystal formation (as further discussed in Sec. 4.5. However, one must keep in mind, that once removed from the cryogenic jet, the yet still cool sample starts to assemble condensed water from the ambient atmosphere. Hence, the sample has to be kept under cryogenic and dry conditions until the very end of all recordings.
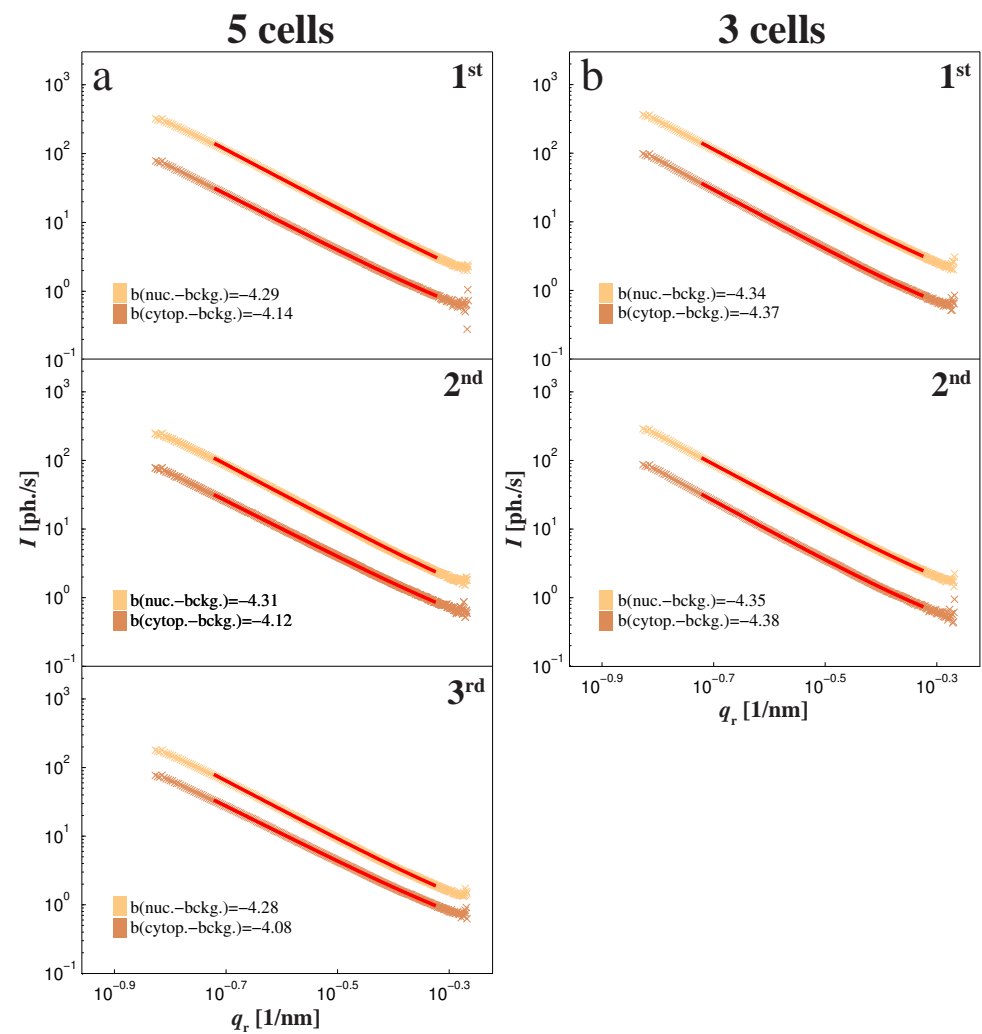

Figure 4.7: (a) Background-corrected radial intensity profiles $I\left(q_{\mathrm{r}}\right)$ for each consecutive scan of two scan series as calculated on the $2 D$ average diffraction patterns (not shown) of the nucleic and cytoplasmic areas defined in Fig. 4.6 (d) and (g). Figure and caption adapted from 42.). 


\begin{tabular}{lllllll}
\hline scan & ROI & run & $\begin{array}{l}\text { a } \\
{[(\mathrm{ph} \cdot \cdot \mathrm{nm}) / \mathrm{s}]}\end{array}$ & $\begin{array}{l}\text { b } \\
{[1]}\end{array}$ & $\begin{array}{l}\text { c } \\
{[\mathrm{ph} / \mathrm{s}]}\end{array}$ & $\begin{array}{l}\mathbf{r}^{\mathbf{2}} \\
{[1]}\end{array}$ \\
\hline 5 cells & cytop.-bckg. & $1^{\text {st }}$ & $3.2 \cdot 10^{-2}$ & -4.14 & $1.5 \cdot 10^{-1}$ & 0.9998 \\
& & $2^{\text {nd }}$ & $3.3 \cdot 10^{-2}$ & -4.12 & $1.3 \cdot 10^{-1}$ & 0.9998 \\
& & $3^{\text {rd }}$ & $3.8 \cdot 10^{-2}$ & -4.08 & $1.7 \cdot 10^{-1}$ & 0.9998 \\
& \multirow{2}{*}{ nuc.-bckg. } & $1^{\text {st }}$ & $1.1 \cdot 10^{-1}$ & -4.29 & $3.0 \cdot 10^{-1}$ & 0.9996 \\
& & $2^{\text {nd }}$ & $8.4 \cdot 10^{-2}$ & -4.31 & $2.9 \cdot 10^{-1}$ & 0.9996 \\
& & $3^{\text {rd }}$ & $6.5 \cdot 10^{-2}$ & -4.28 & $3.2 \cdot 10^{-1}$ & 0.9997 \\
3 cells & cytop.-bckg. & $1^{\text {st }}$ & $2.5 \cdot 10^{-2}$ & -4.37 & $1.7 \cdot 10^{-1}$ & 0.9997 \\
& & $2^{\text {nd }}$ & $2.2 \cdot 10^{-2}$ & -4.38 & $1.6 \cdot 10^{-1}$ & 0.9997 \\
& \multirow{2}{*}{ nuc.-bckg. } & $1^{\text {st }}$ & $1.0 \cdot 10^{-1}$ & -4.34 & $4.9 \cdot 10^{-1}$ & 0.9999 \\
& & $2^{\text {nd }}$ & $7.9 \cdot 10^{-2}$ & -4.35 & $4.4 \cdot 10^{-1}$ & 0.9999 \\
\hline
\end{tabular}

Table 4.1: Fit results for scan series with cryoprotected cells. Table published in 42. 


\subsection{Nano-SAXS from Frozen-Hydrated Cells}

Freeze-drying of a cellular sample reduces the biological specimen to its non-volatile components. At the same time, the lack of water content increases the contrast in each diffraction pattern which provides us with the opportunity to obtain datasets with pronounced scattering signals (as demonstrated in the previous sections). However, preparing a sample in a freeze-dried manner is associated with an invasive preparation protocol, potentially causing major structural artifacts such as shrinkage, detachment or disruptions of cells.
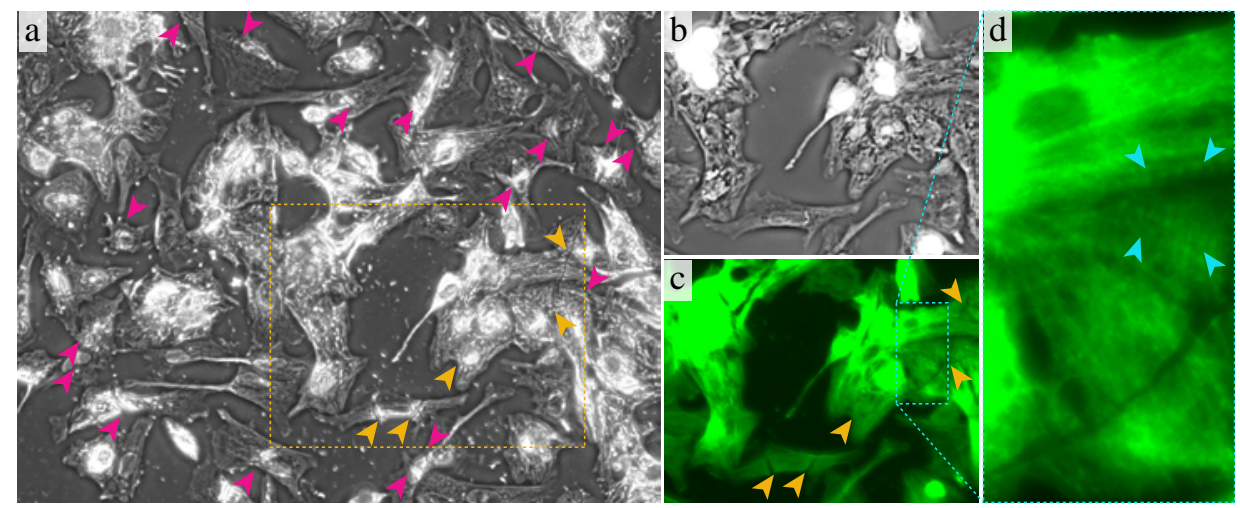

Figure 4.8: (a-b) Visible light phase contrast micrograph of a cellular area of NRCTCs after freeze-drying, see (a), and before plunging the sample, see (b). Pink and orange arrows mark multiple significant cellular disruptions after freeze-drying process. (c-d) Full-field and zoom-in of a visible light fluorescence micrograph of the area shown in (b) after freeze-drying the sample. Orange arrows mark significant cellular disruptions, blue arrows indicate a (preserved) striated fluorescence signal originating from myofibrils.

Fig. 4.8 illustrates structural effects associated with the preparation procedure for a cellular sample (here NRCTCs on a $1 \mu$ m-thick SiRN-window): Fig. 4.8 (a) shows the phase contrast micrograph of the sample after freeze-drying. Several disruptions of cells (pink \& orange arrows) indicate an invasive preparation process, as visualized by comparing a selected area (framed in orange) to the micrograph recorded before plunging, see $(b)$. So far, we have not observed these disruptions in frozen-hydrated cells, implying that they are caused by the last preparative step of freeze-drying. Arguably, these artifacts can be attributed to the degassing effects of volatile components, may it be the (local) sublimation of the buffer or the evaporation of the surrounding liquid nitrogen. (c) Remarkably, the preparative process still preserves the fluorescence signal, as will be further exploited in Sec. 5 (d) A 
closer look shows an adequate preservation of most filamentous structures on the nanoscale, as e.g. can be concluded by the preservation of a striated fluorescence signal (blue arrows) originating from the regular actin arrangements in myofibrils. In order to exclude all structural alterations caused by freeze-drying, one alternative is to vitrify the sample following the same preparative outline except for the invasive last step of sublimating the buffer. This procedure has two main advantages: (i) It preserves the natural structural attributes of the specimen by keeping cells in a (frozen-) hydrated state. (ii) It still reduces the surrounding aqueous layer covering the cell to a minimum (blotting step of the plunging procedure), thus enabling high signal SAXS recordings.

However, handling vitrified samples requires a lot more expertise than handling freeze-dried samples: In order to avoid the formation of ice crystals or an entire defrosting, this approach implies a permanent cryoprotection of the sub-
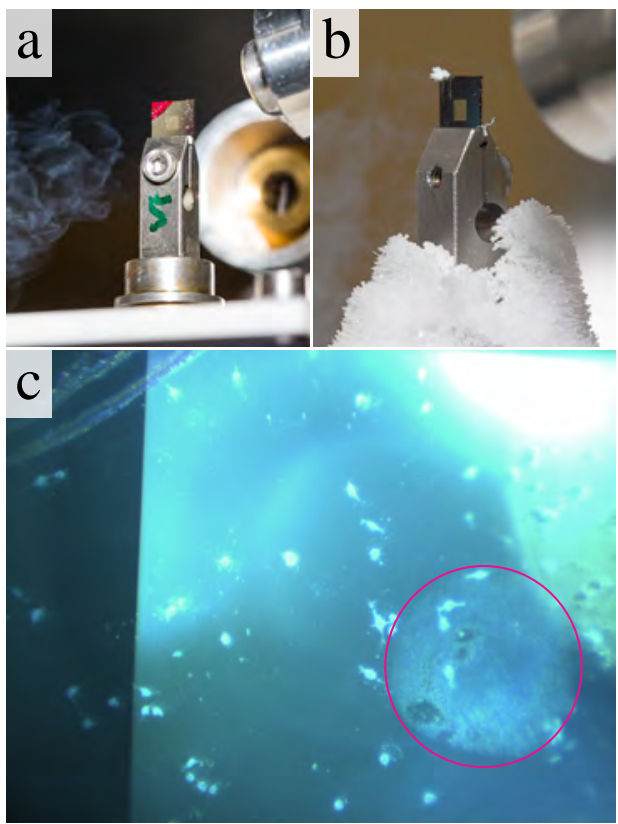

Figure 4.9: $(a-b)$ Photographs of cryoprotected samples. Photos by Dr. M. Osterhoff. (c) OAV-image of a cryoprotected SiRN-window with murine fibroblasts. strate; the associated mounting procedure on the translation stage can thus be quite challenging (see 61) for further details).

Fig. 4.9 illustrates the troubling issue of a successive ice crystal formation showing photographs of (here freeze-dried) samples under cryoprotection, see $(a-b)$, together with an OAV-micrograph of murine fibroblasts on a SiRN-window (as discussed in Sec. 4.4), see $(c)$. An accurate alignment of the sample alongside the cryonozzle is critically important for the success of the experiment, thus ensuring a laminar flow of cryogenic nitrogen, that can fully cover the sample window. A successive accumulation of ice crystals from ambient humidity can easily expand all over the sample (see $(c)$, pink circle) making the following scans useless.

Fig. 4.10 (a) shows the x-ray dark field of nano-SAXS scan performed on a vitrified naive hMSC. Data convincingly show that recording adherent eukaryotic cells in a vitrified state is feasible and worth trying: The cell can be identified clearly showing an intact overall morphology, that is apparently lacking any major disruptions. A sufficiently pro- 
nounced cellular signal further indicates that diffraction patterns can be attributed to nucleic, cytoplasmic and background regions. A closer look on the latter reveals two distinct scattering behaviors of the background which, together with the composite image of (corrected) diffraction data shown in $(b)$, can be attributed to either strong, anisotropic scattering caused by crystalline ice or weak, isotropic scattering originating from an amorphous and thus vitrified aqueous layer.

Arguably, one may point out, that the cellular signal is significantly interrupted by the edges of three separate layers of vitrified buffer. However, this instance at the same time implies an accurate sample preparation procedure indicating that vitrified buffer solution fully covers all the rest of the cell.

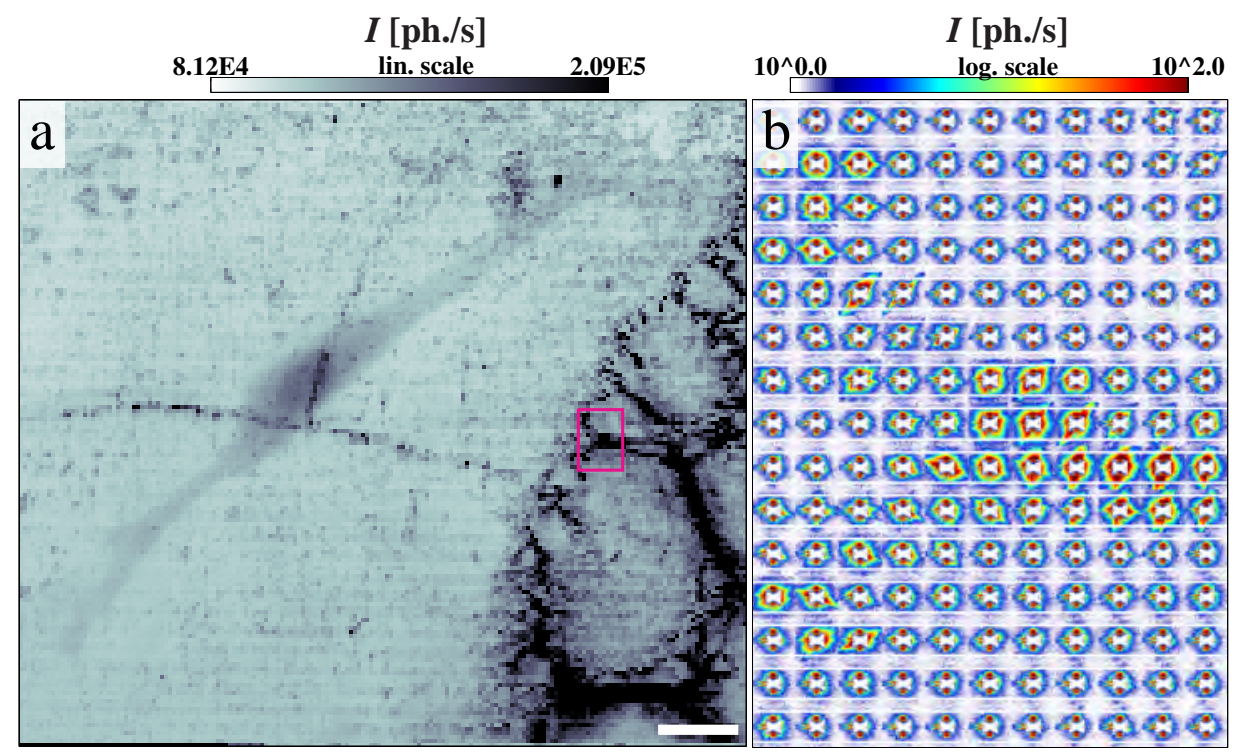

Figure 4.10: (a) X-ray dark-field image of a vitrified naive hMSC. Scale bar: $20 \mu \mathrm{m}$. (b) Diffraction patterns of the area shown in (a) after subtraction of an averaged $2 D$ background signal (not shown). Figure and caption adapted from 42 ). 


\subsection{Scanning SAXS from Hydrated Cells}

Scanning of cells in solution using enclosed chambers enables to circumvent all the artifacts caused by an invasive freeze-drying process or the experimental difficulties encountered on vitrified samples (see Sec.4.5. By using an appropriate highly x-ray transmissive biocompatible chamber, cells can be kept under aqueous - chemically fixated or even alive - conditions when mounted on the sample stage.
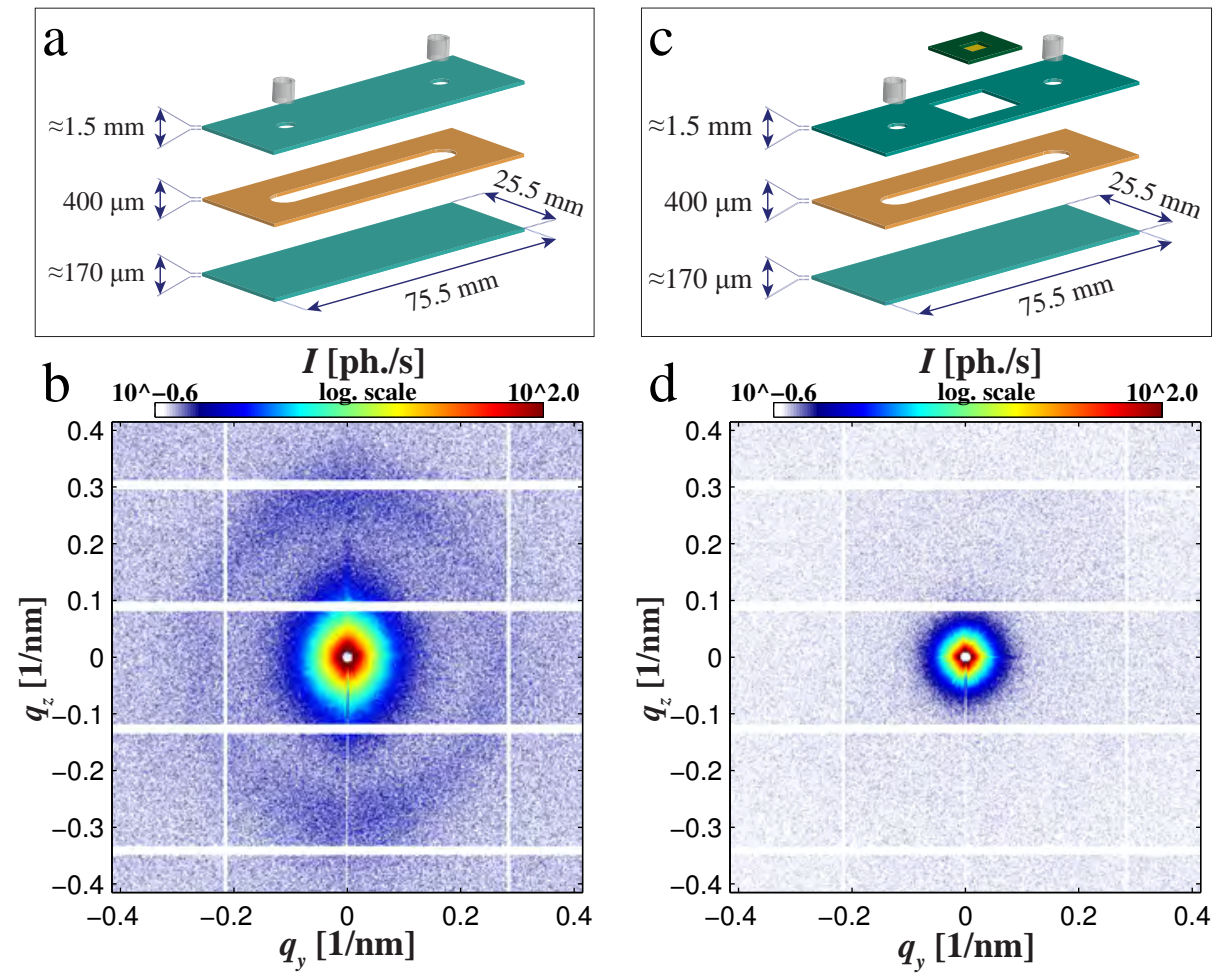

Figure 4.11: left: (a) Sketch of a commercial chamber (ibidi, Germany). (b) The background average diffraction signal of an equivalent 6-channel geometry sample (ibitreat, channel height: $400 \mu \mathrm{m}$, 6-channel cut). right: (c-d) Sketch and background average diffraction signal of a modified chamber with the upper slide replaced by a highly transmissive silicon nitride-window (ibitreat, channel height: $400 \mu \mathrm{m}, 1$-channel cut). Figure and caption adapted from [42], but here showing the average and not the sum of diffraction signals of a background area (for improved clarity).

However, an adequate chamber design fulfilling both physical and biochemical demands is quite challenging: The chamber has to

- provide a surface suitable for cell adhesion. 
- match the specific requirements for buffer recovery during overnight incubation.

- be built of material that allows high x-ray transmission at several photon energies in the (hard) $\mathrm{x}$-ray regime without causing any significant scattering effects.

- provide a sufficiently large reservoir (e.g. by applying a microfluidic flow after cell attachment to the surface) preventing the accumulation of toxic metabolites, while spacing has to be kept to a minimum to avoid significant absorption of the $\mathrm{x}$-ray beam by the buffer.

Previous approaches have addressed these issues based on home-built microfluidic chambers with highly transmissive ultrathin silicon nitride membranes as entry and exit windows (28). This has also led to first nano-SAXS results on (initially) alive cells (29).

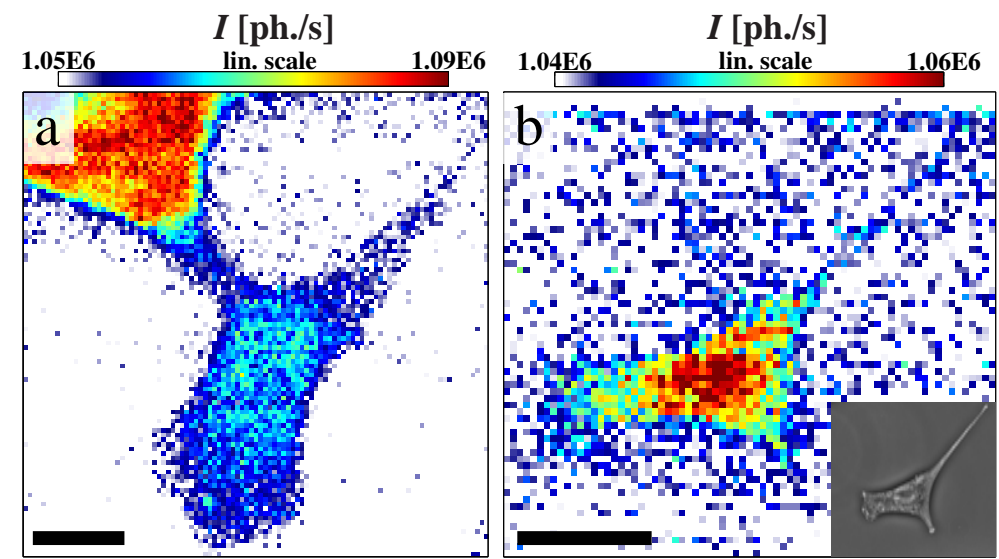

Figure 4.12: $(a-b) X$-ray dark fields of murine myoblasts recorded in a commercially available chamber (ibidi, Germany). (b,inset) Visible light phase contrast image of the cell before recording. Scale bars: $10 \mu \mathrm{m}$ and $20 \mu \mathrm{m}$. Figure and caption adapted from 42 .

Other, commercially available slides primarily made for visible light microscopy (e.g., ibidi, Germany) provide surfaces of various biochemical coatings or physical treatments and allow $\mathrm{CO}_{2}$ diffusion through the bottom polymer for full buffer recovery. Testing these slides has proven them to be x-ray compatible, leading to a satisfactory overall scattering intensity in the x-ray dark field at $E_{\mathrm{ph} .}=13.8 \mathrm{keV}$ and $E_{\mathrm{ph}}=8.7 \mathrm{keV}$, while "the background of the SAXS patterns was found to be too high, despite the fact that the main polymer used is of low residual signal compared to other materials at similar thickness (62)" (42). Fig. 4.11 (a) shows a sketch of a commercially available flow chamber (ibidi, Germany). (b) The background average diffraction signal of an 
equivalent 6-channel geometry recorded at $E_{\mathrm{ph}}=8.7 \mathrm{keV}$ reveal a characteristic peak at $q_{\mathrm{r}} \approx 0.27 \mathrm{~nm}^{-1}$. This feature can be attributed to the upper slide of the channel (with a thickness of $\approx 1.5 \mathrm{~mm}$ ) when comparing the signal to a background average diffraction pattern of a (home-built) modified chamber, see $(c)$ and $(d)$. Here, parts of the upper slide are replaced by a silicon nitride window (of $1 \mu \mathrm{m}$ thickness).

In order to test, if such chambers are also appropriate candidates for a more detailed structural analysis, nano-SAXS experiments are performed on single cells: Fig. 4.12 $(a-b)$ shows the x-ray dark fields of chemically fixated murine myoblasts (at a photon energy of $E_{\mathrm{ph} .}=13.8 \mathrm{keV}$ ) using the same chamber geometry as sketched in Fig. 4.11 (a), but here with a channel height of $200 \mu \mathrm{m}$ and a collagen IV coated bottom layer. While x-ray dark field data occasionally allow to even distinguish between nucleus and cytoplasm (see also visible light micrograph of $(b$, inset)), the cellular diffraction is found to be "too low to perform quantitative analysis on the diffraction data. For such low signals, even the slight intensity variations due to the top-up mode of the storage ring, i. e. the periodic refill of electrons, become noticeable and must be corrected (not shown)" (42).

Another (home-built) chamber design tested in this context consists of two borosilicate glass coverslips with a thickness of $80-130 \mu \mathrm{m}$ and a punched slice of Parafilm forming the volume, see Fig. 4.13 (a). While this 'static' design provides no access by any microfluidic connectors, it is easy to build requiring low expenditure and enables a straightforward cultivation of cells in an open geometry. To this end, the upper coverslip (EMS, USA; edge length cut to $5 \times 5 \mathrm{~mm}$ ) can be transferred into a standard petri dish and then submersed in cell suspension. Glass facilitates cell adhesion even without a previous plasma-cleaning or biochemical coating procedure due to a sufficiently pronounced hydrophilic character of the surface $\rrbracket^{1}$ The durability of the coverslip further allows a segmentation of the sample area (by use of a permanent marker), which is very helpful in identifying individual cell-covered areas via OAV. The chamber is sealed right before measurement allowing fixated or short-lasting alive cell recordings. Fig. 4.13 ( $b-e$ ) shows selected x-ray dark field maps of chemically fixated hMSCs revealing a satisfying signal-to-noise ratio even at a comparatively low photon energy of $E_{\mathrm{ph} .}=7.9 \mathrm{keV}$.

\footnotetext{
1 conversation with Dr. C. Wollnik (04/11/16), DPI, Göttingen; unpublished work of Anna Chizhik et al., DPI, Göttingen
} 


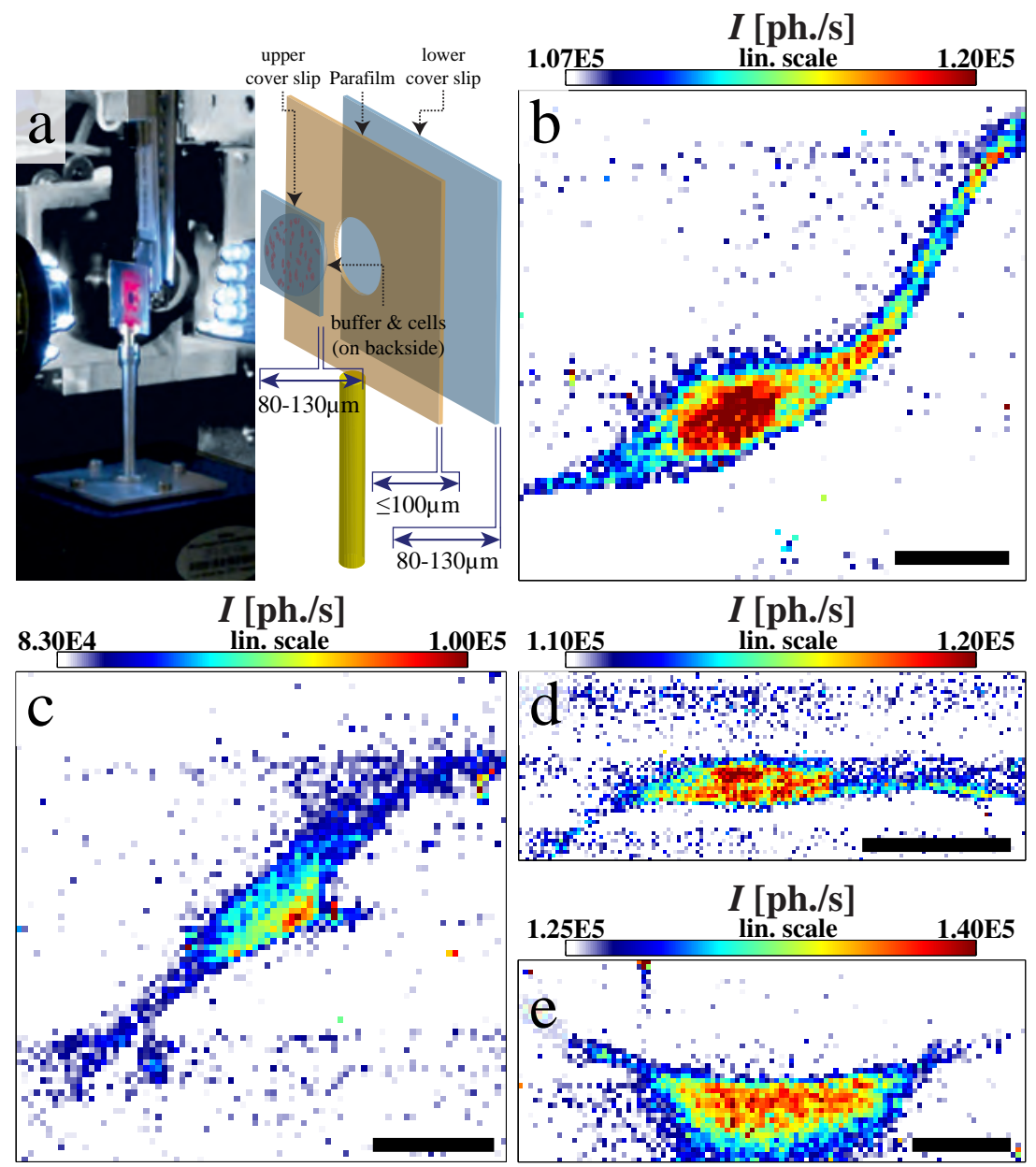

Figure 4.13: (a) Sketch and photograph of a home-built glass chamber. (b-e) X-ray dark fields of selected nanoSAXS scans on chemically fixated hMSCs. Scale bars: $20 \mu \mathrm{m}$. Figure and caption adapted from (42).

In summary, commercially available (modified or unmodified) wet chambers as well as the home-built glass chambers provide a signal-to-noise ratio that allows separation of nucleic and cytoplasmic signals at $E_{\mathrm{ph}}=7.9-13.8 \mathrm{keV}$, but so far do not permit a further characterization of diffraction patterns. However, all chamber geometries have further improved the work flow also revealing some more or less expected features of cells and material: Fig. 4.14 shows two OAV micrographs of a cell while the beam is turned off (see $a$, top) or switched on (see $a$, bottom) revealing a scintillatory effect of the glass visualizing the beam at $E_{\mathrm{ph}} .=7.9 \mathrm{keV}$. This attribute makes glass a con- 
venient window material facilitating the alignment of the instrument and a straight forward tool to address particular sample regions. Notably, the contrast of the (long distance) OAV implemented at GINIX (ACCEL/Bruker, USA; focal length: $\approx 2.8 \mathrm{~cm}$, reflection and transmission illumination, see also Sec.2.1] is indeed sufficient to identify cells in glass chambers during the experiment, which is very important to ensure an acceptable work flow in future projects. Fig. 4.14 (b) shows further OAV micrographs of the cell presented in Fig. 4.13 before (see $b$, top) and after recording (see $b$, bottom) revealing the degassing effect of the beam on the buffer solution. This implies, that alive cell recordings shall be performed in a fully degassed buffer system, making the use of $\mathrm{CO}_{2}$-buffered medium inappropriate (as further detailed in Appendix Sec. 10.5). (c) Coarse scans before (top) and after (bottom) a nano-SAXS fine scan performed on the area (results see Fig. 4.12 a) illustrate the invasive impact of the technique here leading to a complete loss of cellular scattering intensity (see also Sec. 4.4.4.5 for more detail). (d) Inspection of a visible light phase contrast microscopy before (top) and after scanning (bottom) with the results presented in Fig. 4.12 (b) further shows alterations on the substrate surface including a scanning footprint on the area.

The issue of high-contrast hydrated cell recordings will be further addressed in Sec. 5.4 .

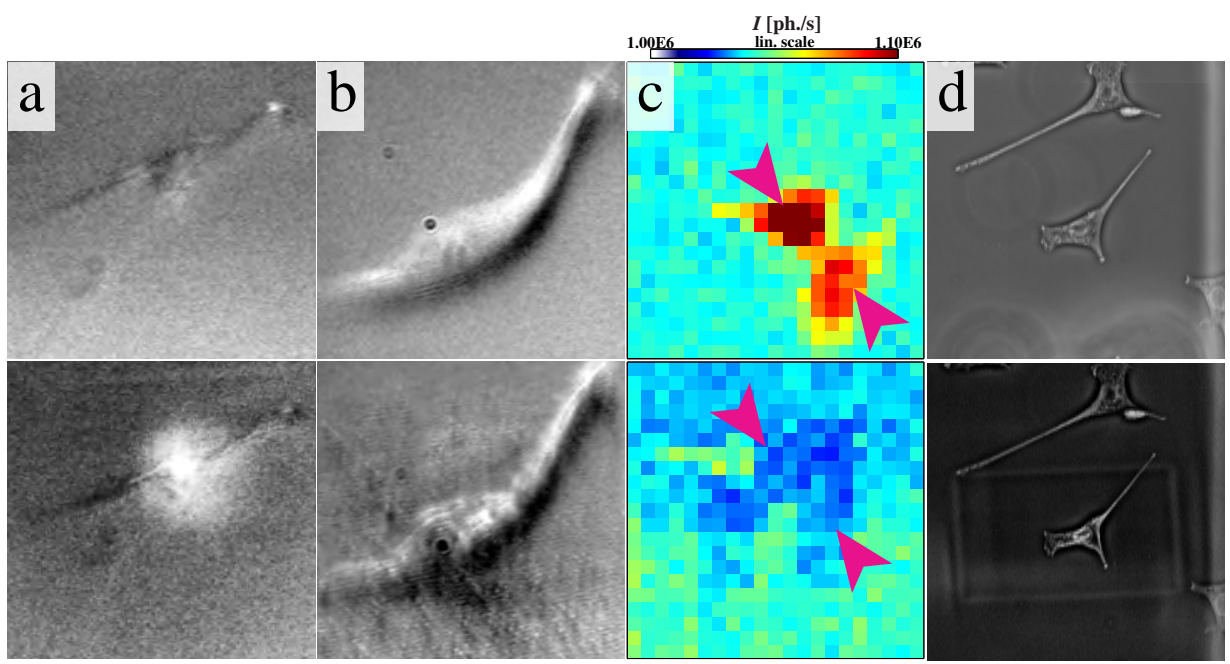

Figure 4.14: (a) OAV micrographs illustrating the scintillatory effect of a glass coverslip. (b) OAV micrographs revealing the degassing effects of the $x$-ray beam on the medium. (c) Coarse scans before and after probing the cells with a finely rastered scan (further results shown in Fig. 4.12 a). (d) Visible light phase contrast micrographs of the cell before and after a SAXS scan (shown in Fig. 4.12 b). 


\section{Section 5}

\section{A SAXS Study of Cardiomyocytes}

\section{This section includes excerpts of (43).}

The recent progress presented in Sec. 4 together with an advanced operating experience in terms of preparative work and instrumentation have enabled to take further steps towards a more comprehensive analysis and interpretation of scanning SAXS data from biological cells.

The present section introduces a study of neonatal rat cardiomyocytes ${ }^{1}$ starting with micro-SAXS scans on freeze-dried samples in Sec. 5.1. Complemented with fluorescence micrographs on actin of the particular region, results allow a first correlative evaluation and an interpretation of data using the comparative analysis approach of Sec. 3.2 Moreover, the respective radial intensity profiles $I\left(q_{\mathrm{r}}\right)$ of the datasets reveal signals with two different power law decays for low- and high- $q_{\mathrm{r}}$-values and thus motivate to apply the robust fitting tool as detailed in Sec. 3.3 Results indicate differences among cells, and by that signify the analytic potential of the algorithm. Large area scans as presented in Sec. 5.2 facilitate a statistical analysis of observables derived from the x-ray dark field, PCA and processed fluorescence data. Sec. 5.3 then focuses on nano-SAXS results revealing isotropic diffraction patterns in the nucleus and patterns with rather pronounced anisotropy in the cytoplasm. PCA is proven to be sufficiently reliable and robust, thus leading to consistent results even when applied on datasets with a non-ideal empty beam profile as well as interrupting detection gaps within the evaluation area. A fluorescence micrograph of the scanned

1 obtained from Luther group; Max-Planck-Institute for Dynamics and Self-Organization, Am Faßberg, 37077 Göttingen 
area further enables a visual inspection of the differences in structure and filament orientation angles $\theta_{\mathrm{fs}} \& \theta_{\mathrm{pa}}$ analogous to previous micro-SAXS results, this time with a much finer scanning step size. Sec. 5.4 closes by presenting first alive cell results.

\subsection{Micro-SAXS on Freeze-Dried Cells}

The results presented in Sec. 3 and Sec. 4 have shown, that the investigation of adherent eukaryotic cell lines by scanning SAXS is feasible, leading to a sufficiently strong cellular signal at several photon energies on samples in multiple preparation states. In particular, diffraction data from freeze-dried samples, where the cellular signal compared to the background is sufficiently high, have revealed systematic anisotropy within the cellular region. This observation has previously been reported as one of the remarkable characteristics of cellular SAXS scans [27, 31, 42, 63, and could be confirmed by follow-up experiments on actin in vitro suspensions as well (32). In order to further inspect the cause of such signals in wild-type eukaryotic cells, where typically actin is the predominant filamentous structure (64,65), this and the following section presents various micro-SAXS scans on freeze-dried NRCTCs. Together with the mathematical processing of the visible light fluorescence micrograph, data are then analyzed correlating the structure orientation (at angle $\theta_{\mathrm{pa}}$ ) as determined on the diffraction patterns to the filament orientation (at angle $\theta_{\mathrm{fs}}$ ) as obtained by visible light fluorescence microscopy. Fig. 5.1 shows the main results of this procedure on a representative dataset comprising (a) the correlative superimposition of x-ray dark field (semitransparent top layer) and fluorescence micrograph (non-transparent bottom layer), (b) the fluorescence micrograph of the cell after freeze-drying, $(c)$ all tracked filaments including the values for the (yet unbinned) orientation angle $\vartheta_{\mathrm{fs}}$ (color code), $(d)$ the local angular deviation $\Delta \theta$ (color-code, as defined in Eq. 3.20 together with $\theta_{\mathrm{fs}}$ and $\theta_{\mathrm{pa}}$ (thin red and bold yellow lines) and ( $e$ ) the histogram of the angular deviation including the mean order parameter $\Omega_{\text {bin }}$ for each bin (color code, see also Eq. 3.21). Pixels in ( $d$ ) that do not include valid values for both orientation angles $\theta_{\mathrm{fs}}$ and $\theta_{\mathrm{pa}}$ (according to thresholded values of both datasets) are marked in white color.

In line with the first results introduced in Sec. 3.2 (see Fig. 3.7. 3.8, data reveal a high (local) correlation between actin filaments and the structure orientation of diffraction patterns indicative for actin being the predominant cause for anisotropic scattering. This becomes clear when regarding both histograms (see Fig. 3.8 ( $g$ ) and Fig. 5.1 (d) ) revealing a narrow cut distribution of angular deviation values $\Delta \theta$ around $0^{\circ}$. Significant 
outliers can be attributed to regions with a rather ambiguous fluorescence signal, $\mathrm{cp}$. (b) \& (d). A closer look at (c) reveals, that micro size beam dimensions cause a broadening of the cellular scattering signal beyond the cell's perimeter, leading to a cellular area that appears to be putatively larger in dark field and PCA maps.

Notably, both cells presented here and in Fig. 3.7 3.8 exhibit anisotropy in all diffraction patterns, that is well-above the background signal, facilitating tracking of cellular structures based on the corresponding $\omega_{\mathrm{pa}}$-values, see $(e$, inset). However, this observation seems not to be a general attribute of NRCTCs, as can be recognized on a larger scan area: Fig. 5.2 shows a respective dataset with the visible light fluorescence micrograph after freeze-drying in $(a)$, the x-ray dark field in $(b)$, the map of PCA results in $(c)$ comprising the structure orientation $\theta_{\mathrm{pa}}$ (blue lines) and the order parameter

\begin{tabular}{ccc}
\hline$p$ & $\min _{p}$ & $\max _{p}$ \\
\hline 0 & $-2.6 \cdot 10^{-1}$ & $9.9 \cdot 10^{-1}$ \\
-1 & $1.5 \cdot 10^{-1}$ & $8.8 \cdot 10^{-1}$ \\
-2 & $-5.8 \cdot 10^{-1}$ & $-4.0 \cdot 10^{-4}$ \\
-3 & $-7.4 \cdot 10^{-3}$ & $1.8 \cdot 10^{-1}$
\end{tabular}
Table 5.1: Color bar limit as used in Fig. 5.3 (c) in units of $\mathrm{nm}^{p} \mathrm{~s}^{p} \mathrm{ph}^{p} /\left(\sum_{p} \mathrm{~nm}^{2 p} \mathrm{~s}^{2 p} \mathrm{ph}^{2 p}\right)^{1 / 2}$. $\omega_{\mathrm{pa}}$ (color code) as well as a composite image based on the center region of the detector in $(d)$. Fig. $5.2(a-b)$ confirm a good agreement between the relative cellular positions (as can be concluded from the fluorescence micrograph) and the occurrence of high scattering intensities (as seen in the dark field). Surprisingly, a comparison of $(b)$ and $(c)$ indicate no explicit correlation between the overall scattering intensity and the anisotropic strength, partially revealing $\omega_{\mathrm{pa}}$-values on cellular positions, which are equal to the background signal. This is a significant difference to the data shown in Fig. 3.7.3.8 and Fig. 5.1] A further inspection of $(b-d)$ reveals the accumulation of anisotropic scattering patterns on the cellular cortex. Arguably, the corresponding anisotropic strength is correlated to the relative fluorescence intensity of the micrograph shown in $(a)$.

Furthermore, the data show characteristic differences among cellular radial intensity curves $I\left(q_{\mathrm{r}}\right)$, exhibiting profiles with single or multiple power law decays: Fig. 5.3 (a) shows the x-ray dark fields of three different NRCTCs comprising a strongly (cell1, $\left.I_{\max }(a, b) \approx 1.5 \cdot 10^{7} \mathrm{ph} . / \mathrm{s}\right)$, a moderately (cell $2, I_{\max }(a, b) \approx 7.5 \cdot 10^{6} \mathrm{ph} . / \mathrm{s}$ ), and a rather weakly scattering cell (cell3, $I_{\max }(a, b) \approx 3.7 \cdot 10^{6} \mathrm{ph}$./s). Fig. 5.3 (b) shows the corresponding local radial profiles as obtained by (full) azimuthal integration on four selected single spots (as also marked in Fig.5.3 (a), (1)-(4)): 

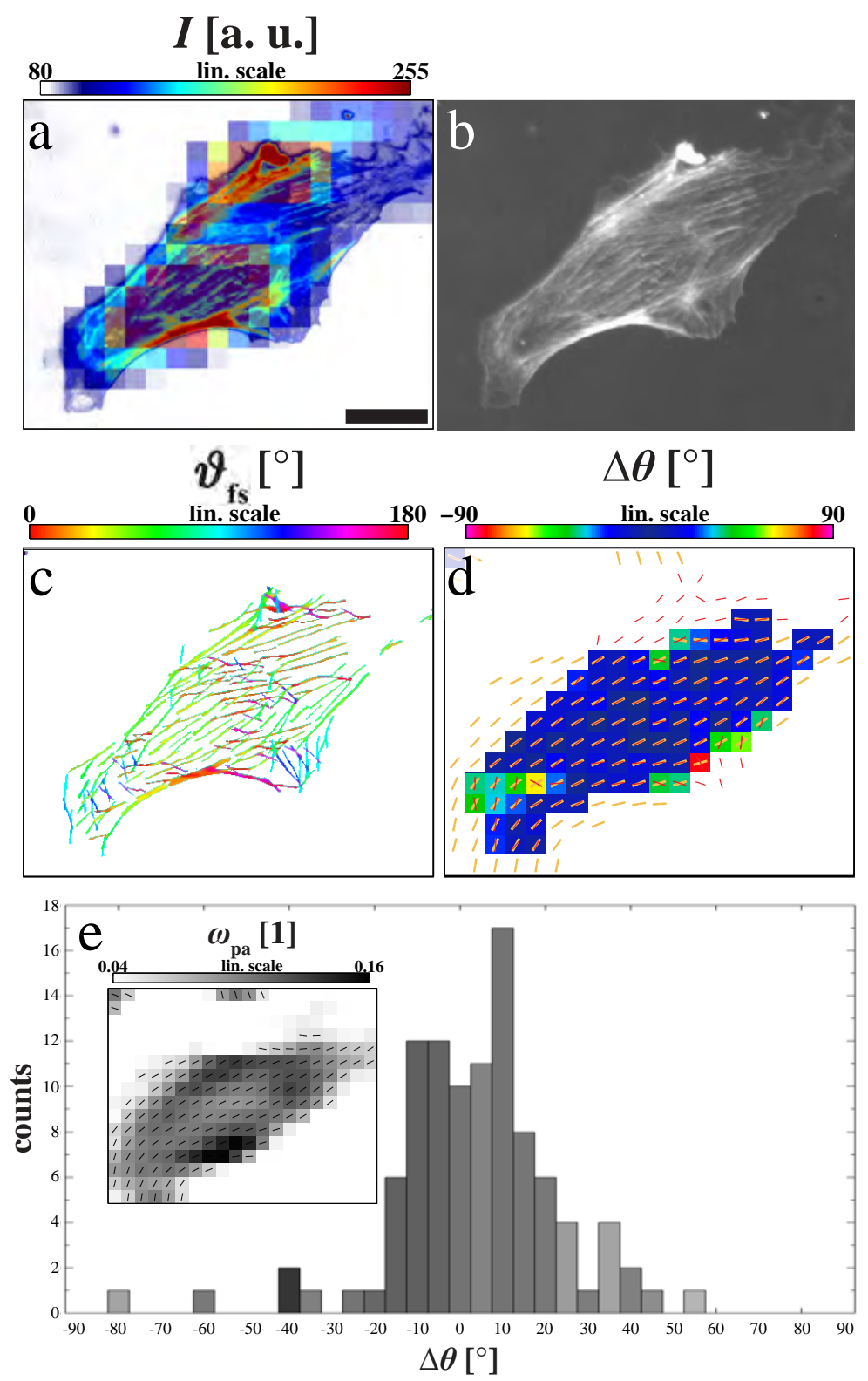

Figure 5.1: (a) Correlative superimposition of x-ray dark field (semitransparent top layer) and fluorescence micrograph (non-transparent bottom layer). Scale bar: $40 \mu \mathrm{m}$. (b) Fluorescence micrograph of the cell after freeze-drying. (c) Tracked filaments as found on the fluorescence image after freeze-drying showing values for the orientation angle $\vartheta_{\mathrm{fs}}$ as color code. (d) Local angular deviation showing $\theta_{\mathrm{fs}}$ and $\theta_{\mathrm{pa}}$ depicted as thin red and bold yellow lines and $\Delta \theta$ as color code. (e) Histogram of the angular deviation including the mean order parameter $\Omega_{\text {bin }}$ for each bin as color code (see Eq. 3.21 together with PCA results on the scan area (inset). 
Signals (1) (of cell1) and (2) (of cell2) reveal two differing decays for low- and high- $q_{\mathrm{r}^{-}}$ values recognizable as a 'kink' in the middle of the data curve. Contrarily, profiles (3) (of cell3) and (4) (background signal) show rather constantly descending signals. This diversity cannot be described adequately well by a (simple) power-law decay (see Eq. 2.5. unless one subdivides the signal into low and high $q_{\mathrm{r}}$-regions [29]. However, replacing this power-law function by a linear combination of decays with fixed integer exponents allows an adequate description of all local profiles shown here in a batch process (as detailed in Sec. 3.3. Fig. 5.3 shows the respective fit results on all four profiles (pink lines) following Eq. 3.22 with fixed limits $z_{\text {high }}=0$ and $z_{\text {low }}=-8$.

This empirical approach reduces the tremendous amount of data (each profile may very well consist of more than 1200 data points) to nine observables describing the scan area: Fig. 5.3 “' $(c)$ shows the normalized values of the first four coefficients $c_{0}^{*}(a, b)$ $c_{-3}^{*}(a, b)$ with

$$
c_{p}^{*}(a, b)=\frac{c_{p}(a, b)}{\left(\sum_{p} c_{p}^{2}(a, b)\right)^{1 / 2}}
$$

as a measure for the relative contribution of each coefficient $c_{p}$ to the fit" 43) (color bar limits: see Tab.5.1. Fig. $5.3(d)$ shows the absolute values for $c_{-1}^{*}$ to $c_{-4}^{*}$, considering all diffraction patterns within the regions $\mathrm{a}_{1}-\mathrm{a}_{4}$ (see $(a)$, hatched areas), on a logarithmic scale. Both subfigures $(c)$ and $(d)$ indicate a relatively consistent behavior among cell1 and cell 2 while values for cell 3 vary to a large extent (even causing changes of the algebraic sign for $c_{-3}^{*}$ - and $c_{-4}^{*}$-values, see Fig. $5.3(d)$ ). This circumstance may be attributed to structural differences between these cells.

Unlike some model systems (27, 32, 63) the scanning SAXS data presented here reveal no modulations in neither the $2 \mathrm{D}$ signal nor the $1 \mathrm{D}$ azimuthal average, making radial intensity profiles much easier to describe, but much harder to assign to specific structural geometries. The fitting approach proposed here reliably follows the decays of unmodulated signals in an automated manner, but also make it possible to determine the overall decays of modulated signals as well. Decays of the radial intensity profile in theory can be traced back to (simple or more complex) real space geometries such as spheres, discs and rods [26), while simulations may lead to benchmarking $c_{p}$-values for relevant model geometries. With simulated model-based diffraction data on the one hand (bottom up approach) and real cellular data on the other (top down approach) the proposed fitting approach could identify characteristic features in the diffraction patterns which can then be linked to form- and structure factors of the sample's local constituents. 


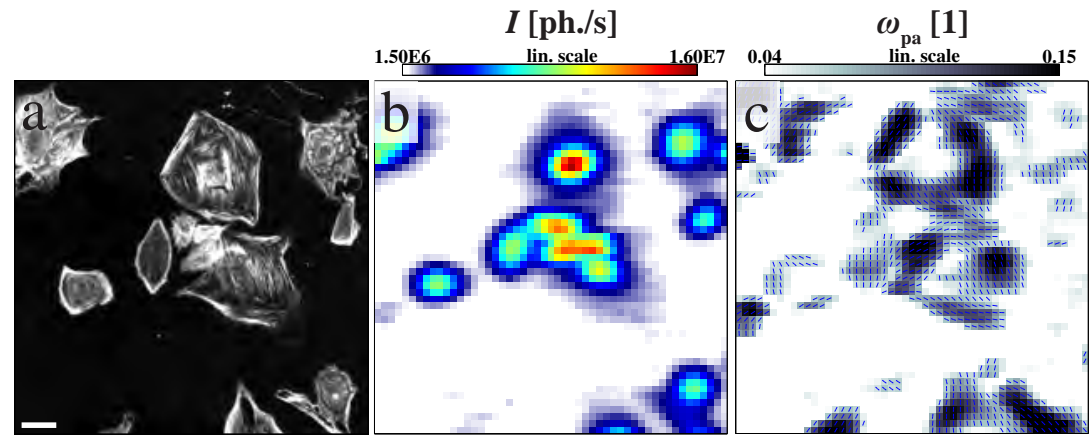

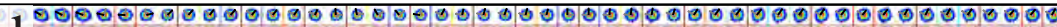

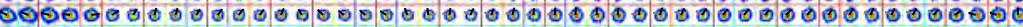

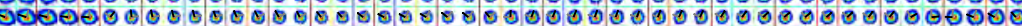

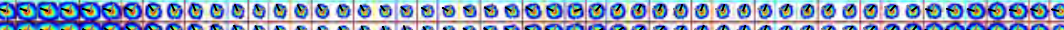

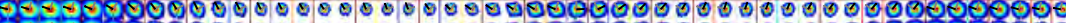

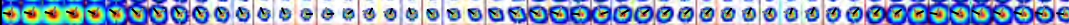
- t r r

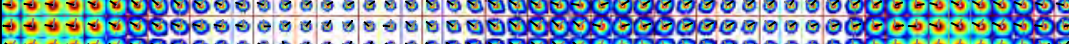
r r r r

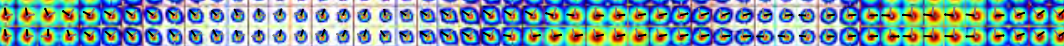

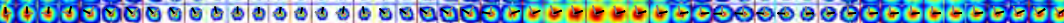

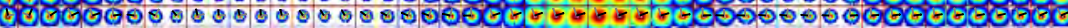

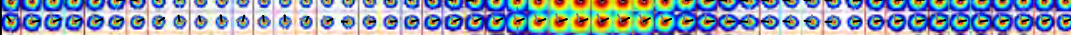

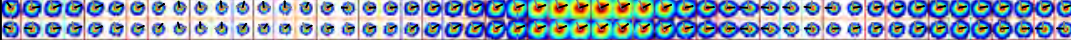

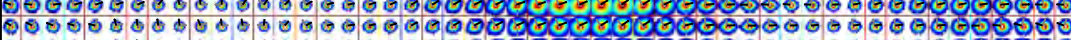

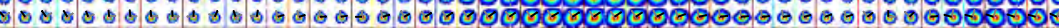

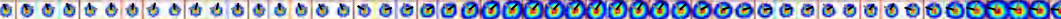

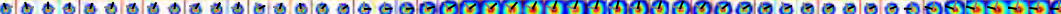
- 0 a 000 क

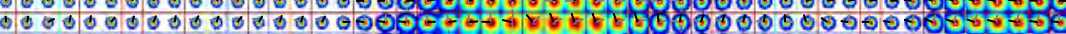

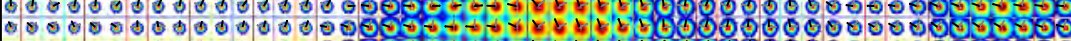

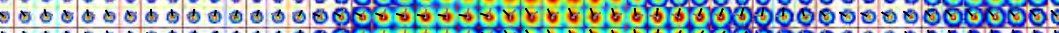

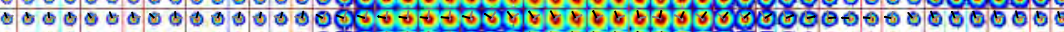

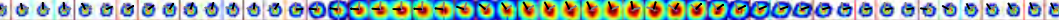

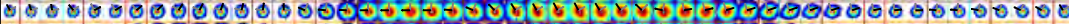

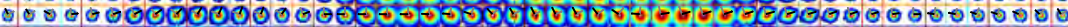

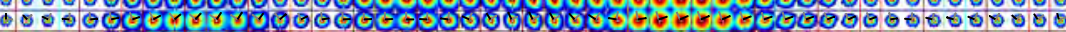

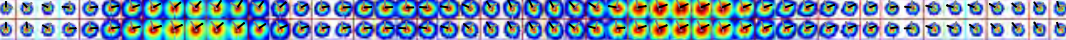

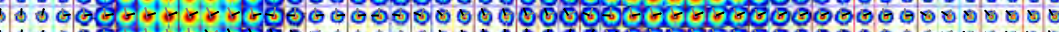

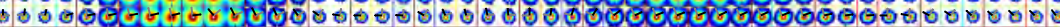

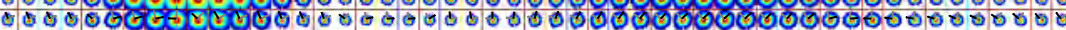

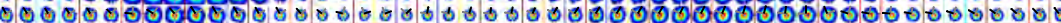

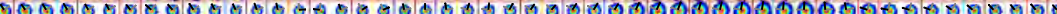
-

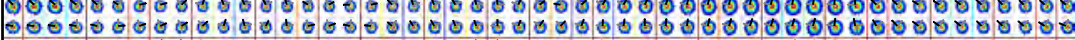

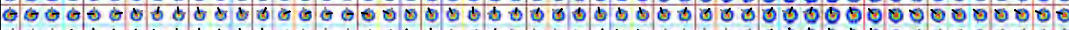

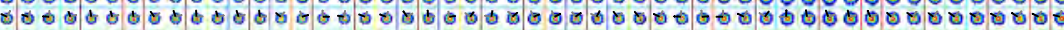

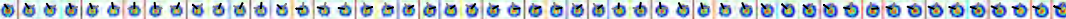
000000000000000000000000000000000000000000001

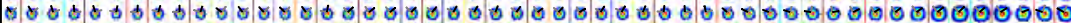

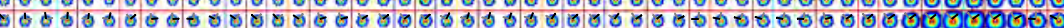
ob

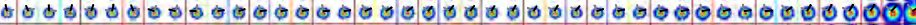

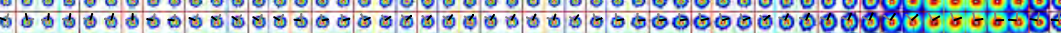

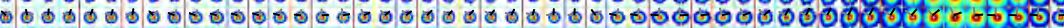

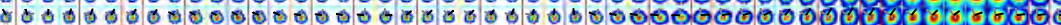

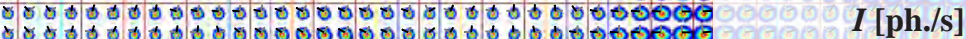

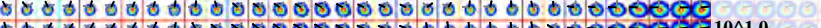

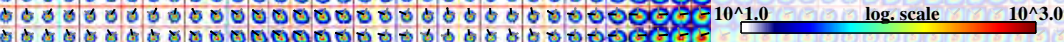

Figure 5.2: (a) Fluorescence micrograph of the micro-SAXS scan area shown in (b-d). Scale bar: $50 \mu m$. (b) Xray dark field. (c) PCA result showing the filament orientation $\theta_{\mathrm{pa}}$ (blue lines) and the anisotropy parameter $\omega_{\mathrm{pa}}$ (color code). (d) Composite image showing the central part of each diffraction pattern and the rescaled principal axes $\vec{l}_{k}, k \in[1,2]$, defined in Eq. 3.8 Figure and caption adapted from (43). 

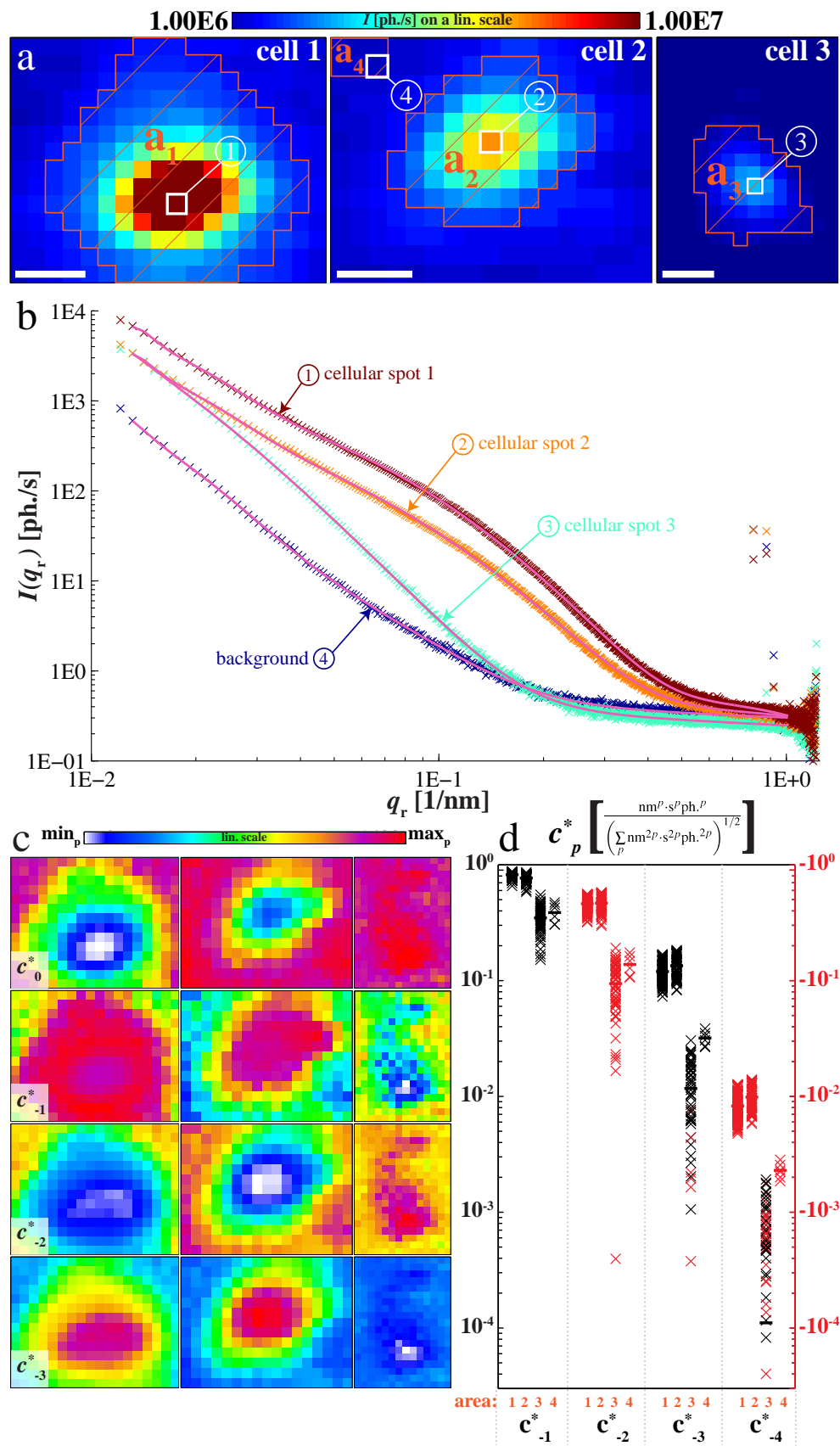

Figure 5.3: (a) X-ray dark fields of three NRCTCs. Scale bar: $40 \mu \mathrm{m}$. (b) Intensity profiles of selected single spots as marked in a including fitting results (pink lines) on the basis of $I_{\mathrm{auto}}\left(q_{\mathrm{r}}\right)$ as defined in Eq. 3.22 with fixed limits $z_{\mathrm{high}}=0$ and $z_{\mathrm{low}}=-8$. (c) Normalized coefficients $c_{-4}^{*} c_{-1}^{*}$ of the three scan regions shown in a as defined by Eq.5.1. Color bar limits are listed in Tab.5.1] (d) Statistical analysis of $\left|c_{-4}^{*}\right|-\left|c_{-1}^{*}\right|$ of the hatched areas $a_{1}-a_{4}$ as marked in a. Black and red crosses denote positive or negative $c_{p}^{*}(a, b)$-value, gray bars mark the respective average values. Figure and caption adapted from [43). 


\subsection{Increasing the Statistics by Large Area Scans}

The special attributes of the micro-SAXS setup (as discussed previously) enable large area scans in an affordable amount of beam time. Presupposing an adequate cell density within the scan region, this instance can be further taken advantage of in order to address cellular structural attributes of cells with little effort in a statistical manner. This section focuses on further establishing the comparative analysis results of Sec.5.1. before evaluating NRCTCs with respect to the observables $\langle I\rangle, \Omega, s$ and $\langle\bar{\sigma}\rangle$ as defined in Sec.4.2

Fig. 5.4 (a) shows the visible light fluorescence micrograph of an entire SiRN-window (edge length: $1.5 \mathrm{~mm}$ ) with a moderate density of freeze-dried NRCTCs. The data indicate an adequate preservation of macroscopic cellular structures in the freeze-drying process, only occasionally revealing cellular disruptions. Filaments are tracked leading to the orientation angles $\vartheta_{\mathrm{fs}}(\tilde{y}, \tilde{z})$ as shown in $(b)$. Five successive micro-SAXS scans are performed probing the (approximated) area as framed by colored rectangles. The respective dark fields are merged ('by eye', with an estimated accuracy of one scanning step size $\Delta=10 \mu \mathrm{m}$ ) leading to x-ray dark field results as shown in (c) and PCA results as shown in $(d)$. Structure orientation angles $\theta_{\mathrm{pa}}$ of $(d)$ thresholded to $\omega_{\mathrm{pa}}$-values above 0.028. Comparison of $(c)$ and $(a)$ convincingly show that all cells of the fluorescence image are also traceable in the x-ray dark field map and exhibit maximum scattering intensities in the cellular center on a regular basis. These intensities however appear to differ among cells, possibly indicating the presence of more than one cell type on the sample plane. PCA results of $(d)$ further reveal a background signal that is sufficiently isotropic, leading to vast background regions with relatively low $\omega_{\text {pa }}$-values (typically smaller than 0.02). However, these results are also partially inconsistent, in the sense that they sometimes reveal relatively high anisotropies in background regions. This can possibly be attributed to the coating layer of the membrane. Together with an irregular cellular signal (as discussed in Sec. 5.1], this prevents to reliably track cells according to their $\omega_{\mathrm{pa}}$-values. Fig. 5.4 ( $e$ - $f$ ) show the corresponding superimposed image of the $\mathrm{x}$-ray dark field (semitransparent top layer) and the visible light fluorescence image (non-transparent bottom layer) following the same analysis outlines detailed in Sec. 3.2. Data imply occasional differences between the fluorescence image and the x-ray dark field, sometimes leading to dimensional mismatches for particular cells. 

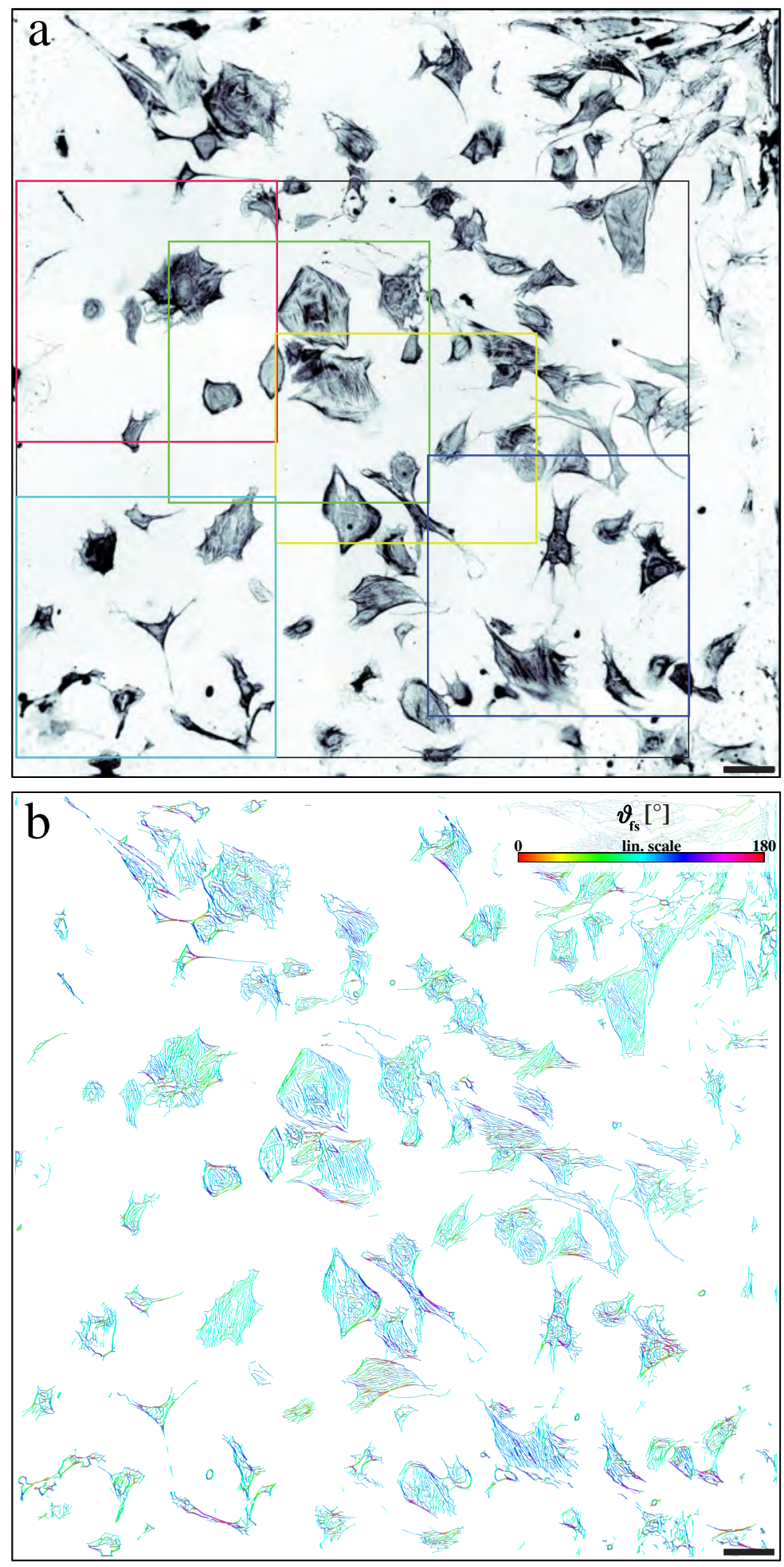

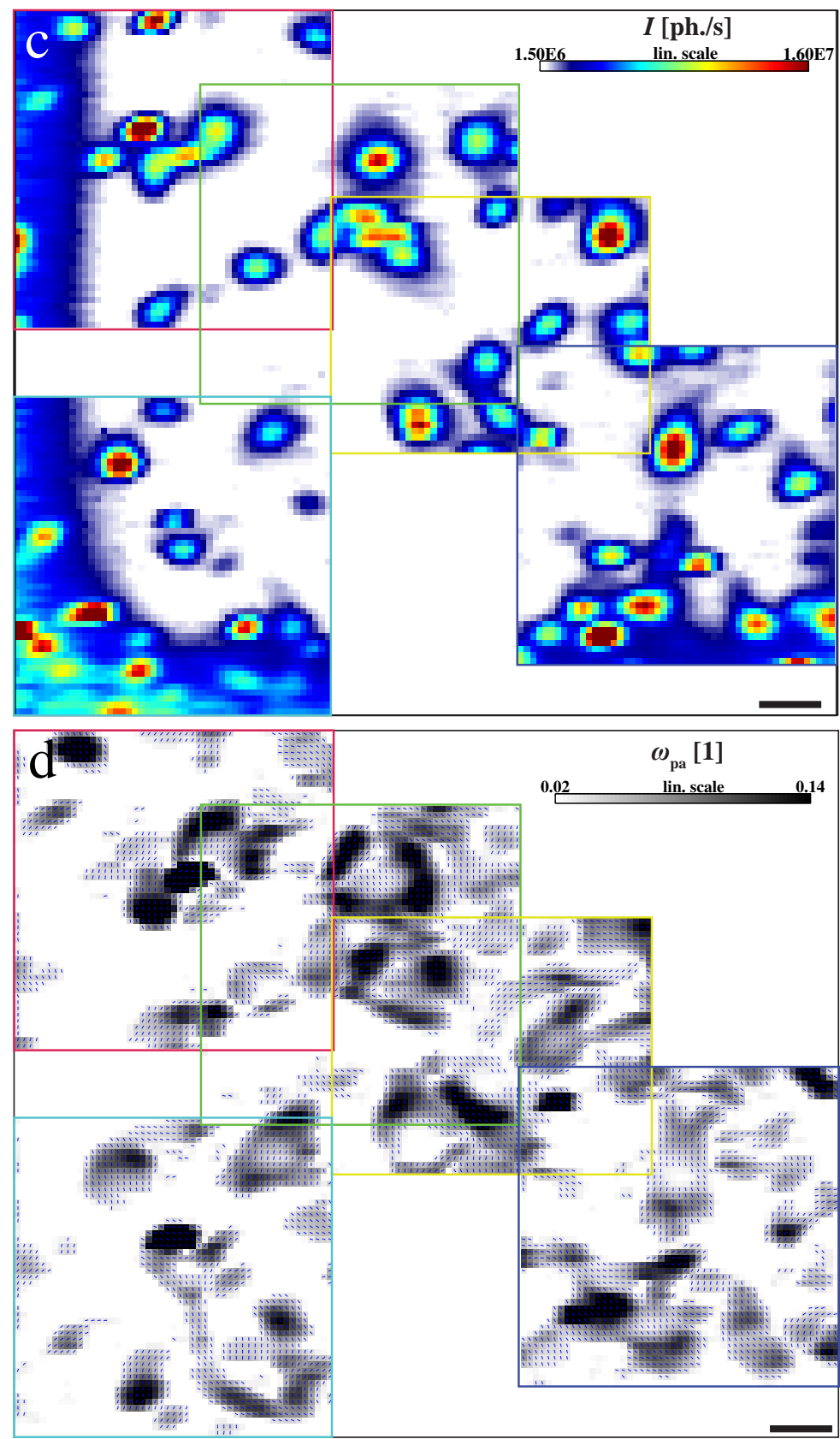

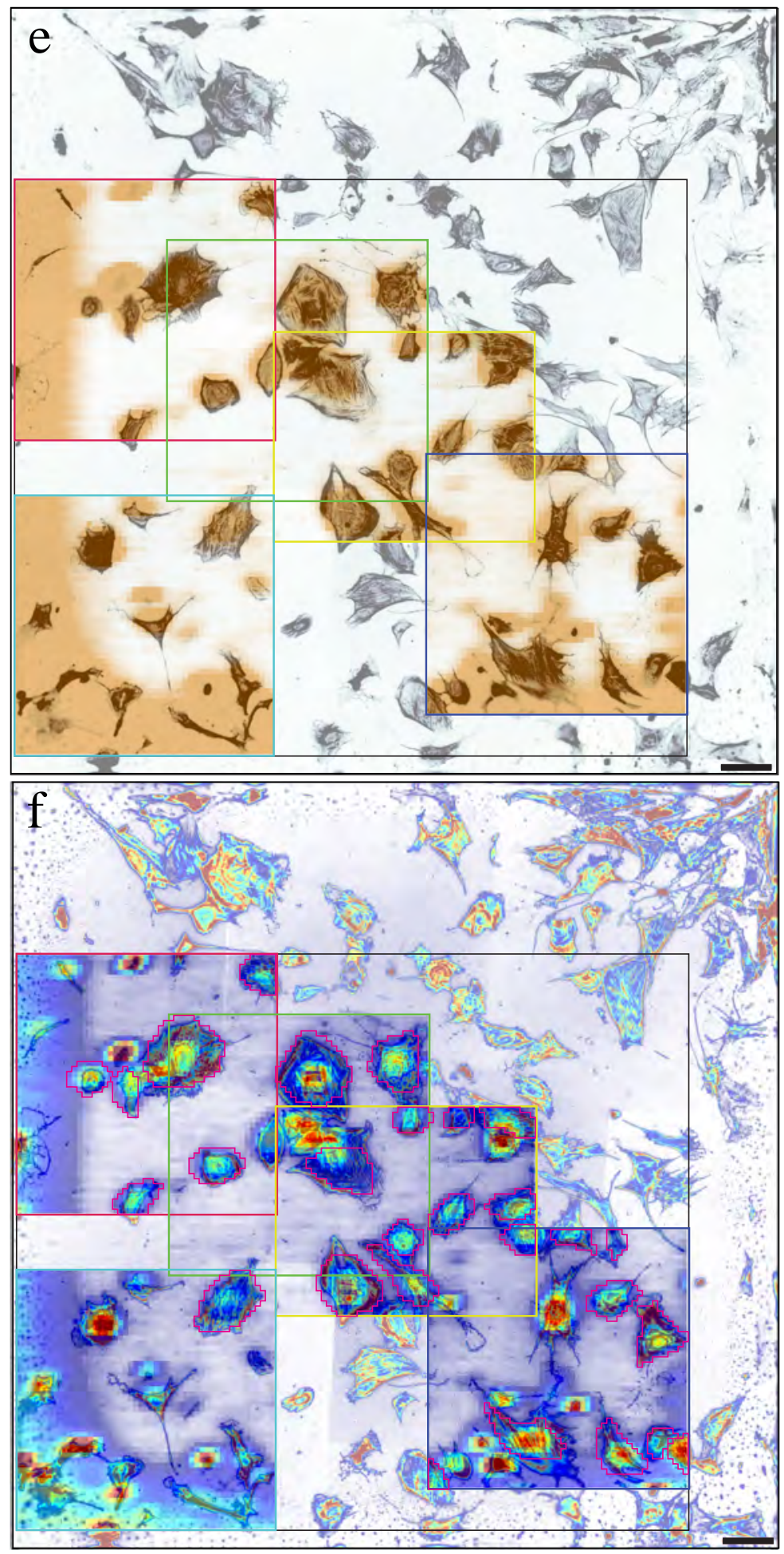


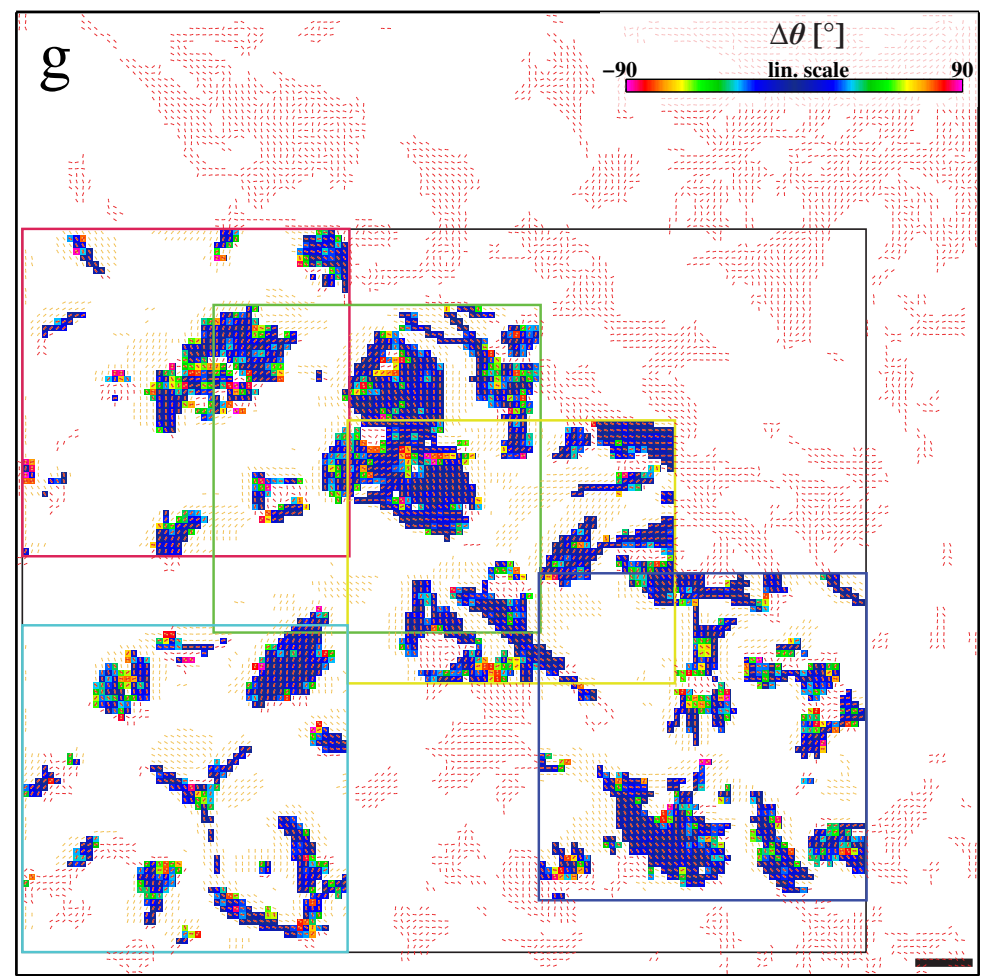

Figure 5.4: (a) Fluorescence micrograph of the entire sample area of an ultrathin SiRN-window (edge length: $1.5 \times 1.5 \mathrm{~mm}$ ) depicted in false color. Colored rectangles mark the approximated scan positions of the five scans, following the comparative analysis procedure detailed in Sec. 3.2 (main article). (b) Unbinned orientation angles $\vartheta_{\mathrm{fs}}(y, z)$ for each pixel of the fluorescence image as found by the filament sensor (51). White areas contain no trackable filament. (c) Merged x-ray dark fields of all five scans. (d) Merged PCA results showing the orientation angles $\theta_{\mathrm{pa}}(a, b)$ (blue lines) and the order parameter $\omega_{\mathrm{pa}}(a, b)$ (color code). (e-f) Correlative superimposition of the $x$-ray dark field (semi-transparent top layer) and the unbinned fluorescence micrograph (non-transparent bottom layer). (g) $\theta_{\mathrm{fs}}(a, b)$ (thin red lines) \& $\theta_{\mathrm{pa}}(a, b)$ (bold yellow lines) together with the angular deviation $\Delta \theta(a, b)$ (color code) as defined by Eq. 3.20 In the white areas, no value for $\Delta \theta(a, b)$ can be determined. All scale bars: $100 \mu \mathrm{m}$. Figure and caption adapted from 43.

For a further statistical analysis determining the generalizing observables $\langle I\rangle, \Omega, s$ and $\langle\bar{\sigma}\rangle$ (see Sec. 4.2 with results shown further below), cellular masks have therefore to be well-selected and -adjusted taking the fluorescence micrograph as a basis (see $(e)$, pink frames). Fig.5.4 ( $g$ ) shows the local angular deviation $\Delta \theta$ (color-code) together with $\theta_{\mathrm{fs}}$ (thin red lines) and $\theta_{\mathrm{pa}}$ (bold yellow lines). Data confirm previous observations of an agreement between both orientation angles. 


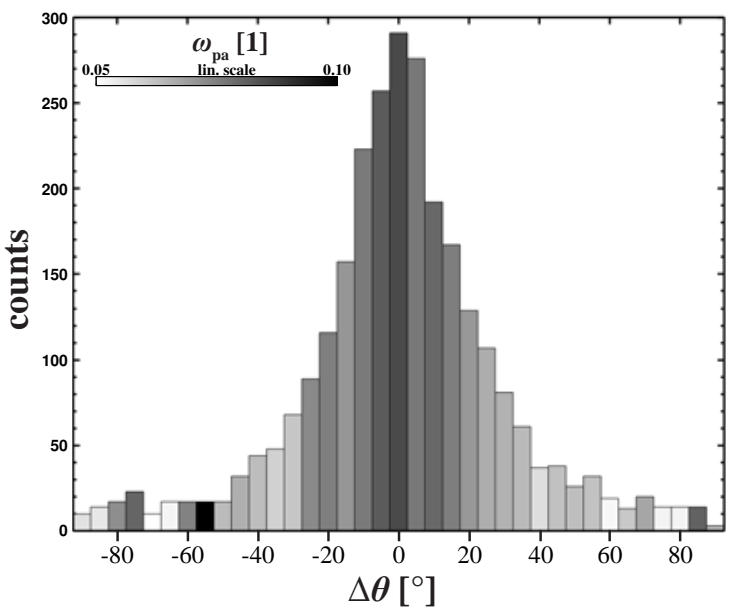

Figure 5.5: Histogram of the angular deviation $\Delta \theta$ as defined by Eq. 3.20 Results include averaged values for the order parameter $\omega_{\mathrm{pa}}$ as quantified by PCA (color code). Figure and caption adapted from [43).

A corresponding histogram of angular distributions $\Delta \theta$, see Fig. 5.5 reveals a global maximum at $\Delta \theta=0^{\circ}$ and a strongly descending signal towards $|\Delta \theta|=90^{\circ}$. The large amount of evaluated data points indicate a statistical correlation between actin filaments and the x-ray structure orientation. This correlation seems to be $\omega_{\mathrm{pa}}$-dependent, as one can derive from the mean values $\Omega_{\text {bin }}$ for each bin (color code, see also Eq. 3.21 ). One possible interpretation is, that only sufficiently pronounced anisotropic scattering signals allow an accurate determination of $\theta_{\mathrm{pa}}(a, b)$, thus leading to coinciding results for $\theta_{\mathrm{fs}}$ and $\theta_{\mathrm{pa}}$. Finally, we see that visible light fluorescence and x-ray data differ to a certain extent, indicative for other, unlabeled local structures present at the probing spots.

Following the analysis scheme introduced in Sec. 4.2 Fig. 5.6 shows results for the generalizing observables $\langle I\rangle$ (in $(a)$ ), $\Omega$ (in $(b)$ ), $s$ (in $(c)$ ) and $\langle\bar{\sigma}\rangle$ (in $(d)$ ) as obtained from selected NRCTCs (Fig. 5.4 ( $e$, pink frames)). $(a-b)$ Cells show a moderate overall scattering intensity on the level of naive hMSCs and a rather poor anisotropy. Both observations can be well-understood when comparing x-ray dark field and PCA results shown in Fig. 5.4 to the respective datasets of the other cell lines (see Sec. 4.1). Arguably, values for $\Omega$ seem to dissect into two distinct data assemblies, which may be interpreted as a structural difference among cells. (c) Results for the nematic order parameter $s$ further show, that the order of filamentous structures in NRCTCs seems to be quite diverse, which is in good agreement with the fluorescence micrograph of the sample (see Fig.5.4 $(a-b)$ ). (d) Remarkably, NRCTCs appear to have the highest mean standard deviation of the momentum transfer $\langle\bar{\sigma}\rangle$. This can be traced back to the previously 
discussed attributes in the radial intensity profiles, which has effectively shifted data towards higher $q_{\mathrm{r}}$-values. Note, that the NRCTCs shown here were chemically fixated before plunging and may thus not be directly comparable to the other cell lines.

In conclusion, one may recognize, that all scans of Fig. 5.4 have been recorded within one morning proving that well-established statistics of single cell observables is indeed feasible with the approaches developed here.
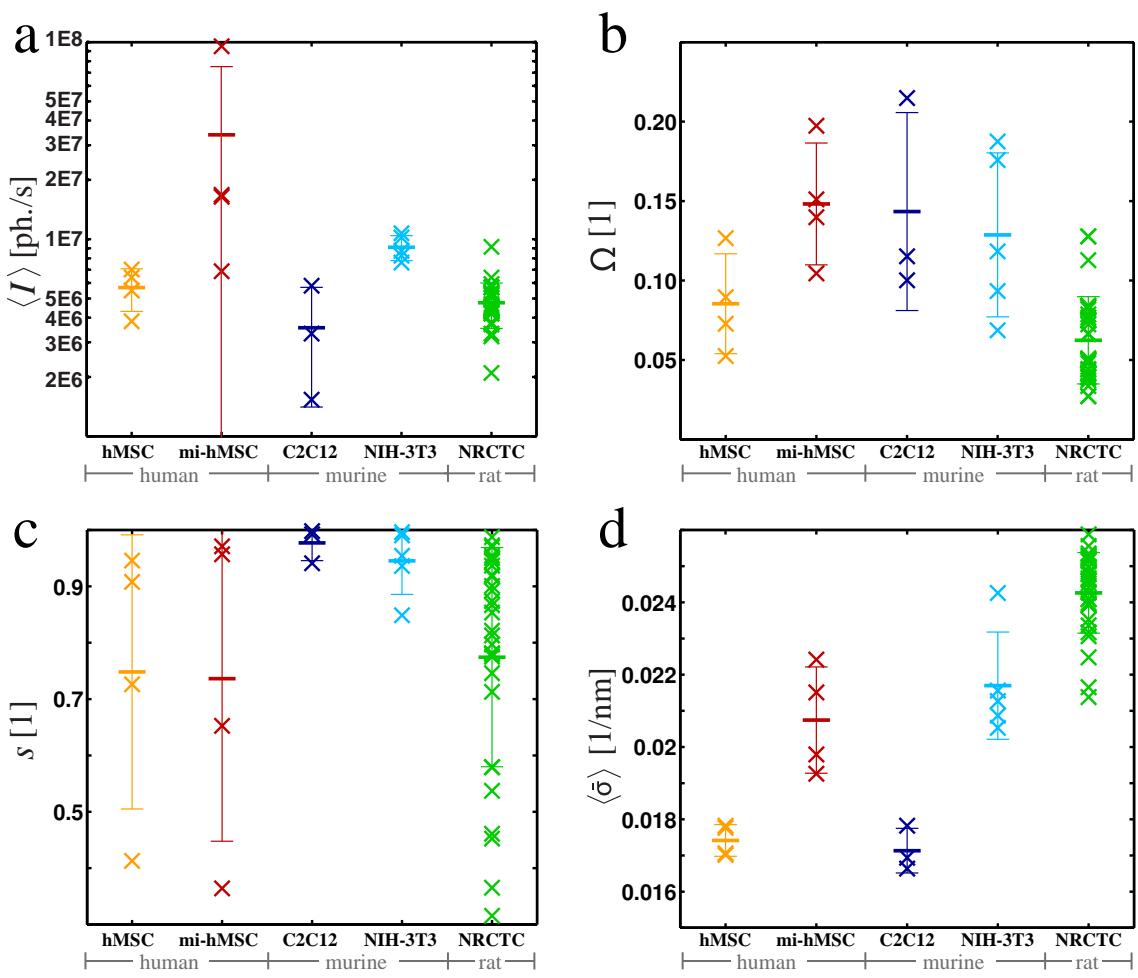

Figure 5.6: (a) mean scattering intensity $\langle I\rangle$, (b) the mean order parameter $\Omega$, (c) the $2 D$ nematic order parameter $s$ and $(d)$ the mean standard deviation of the momentum transfer $\langle\bar{\sigma}\rangle$. Figure and caption adapted from 42). 


\subsection{Nano-SAXS on Cardiac Tissue Cells}

In this section we present scanning nano-SAXS experiments providing an opportunity to further elaborate structural details of NRCTCs with a significantly enhanced real space resolution (see also Sec. 2.2.1 2.2.2]: Fig. 5.7 shows a representative nanoSAXS scan on freeze-dried NRCTCs under cryogenic protection, probed with a step size $\Delta=0.5 \mu \mathrm{m}$. ( $a$, left) The x-ray dark field reveals several cells within the scan area enabling us to distinguish between nucleic, cytoplasmic and background regions. Results indicate, that in particular nucleic regions differ in their overall scattering strength, confirming previous observations of Sec. 5.2 ( $a$, right) exemplarily shows directly neighboring diffraction patterns of four individual cellular areas (1)-(4): Nucleic diffraction patterns reveal rather isotropic but intense signals, see (1), leading to principal axes $\vec{l}_{k}$ of almost equal length (see Eq. 3.8. and low $\omega_{\mathrm{pa}}$-values. Contrarily, diffraction patterns originating from (certain) cytoplasmic regions exhibit a pronounced anisotropic but less intense behavior, see (2)-(4). This observation is very much in line with the nanoSAXS results on stem cells (see Sec. 4.3, but at the same time anisotropy of selected spots appears to be much more pronounced (cp. to Fig. $4.5 d$ ). Fig. 5.7 (b) shows the fluorescence image of the sample area before plunging, facilitating the tracking of most significant filaments, the results for $\theta_{\mathrm{fs}}$ are presented in (c).

Sufficiently high $\omega_{\mathrm{pa}}$-values for cellular diffraction patterns allow masking of the cell by thresholding structure orientation angles $\theta_{\mathrm{pa}}$ to $\omega_{\mathrm{pa}}>0.04$, see $(d)$. Comparison to filament orientation angles $\theta_{\mathrm{fs}}$ of $(c)$ reveal a significant consistency among data confirming the previous findings of Sec.5.1 5.2 Fig. 5.7 (e) shows corresponding $\omega_{\mathrm{pa}}$-values for each diffraction pattern. Comparison to the micrograph of $(b)$ reveal a correlation between $\omega_{\text {pa }}$ and the relative fluorescence intensity. Fig. 5.7 $(f)$ shows PCA results showing $\omega_{\mathrm{pa}}$ as color code and structure orientation angles $\theta_{\mathrm{pa}}$ thresholded to $\omega_{\mathrm{pa}}$-values above 0.04 as black lines. For presentation purposes, only every third diffraction pattern is shown. Data indicate a high correlation among neighboring diffraction patterns, that is predominantly found in the long cellular extensions and on the perimeter, and can be further quantified by use of the nematic order parameter $s$ : To this end, $s(a, b)$ at position $(a, b) \in \mathbb{N}^{2}$ is calculated for each scan point, considering an area of $3 \times 3$ diffraction patterns (direct neighbors). The corresponding mask is then successively translated throughout the entire scan area. 


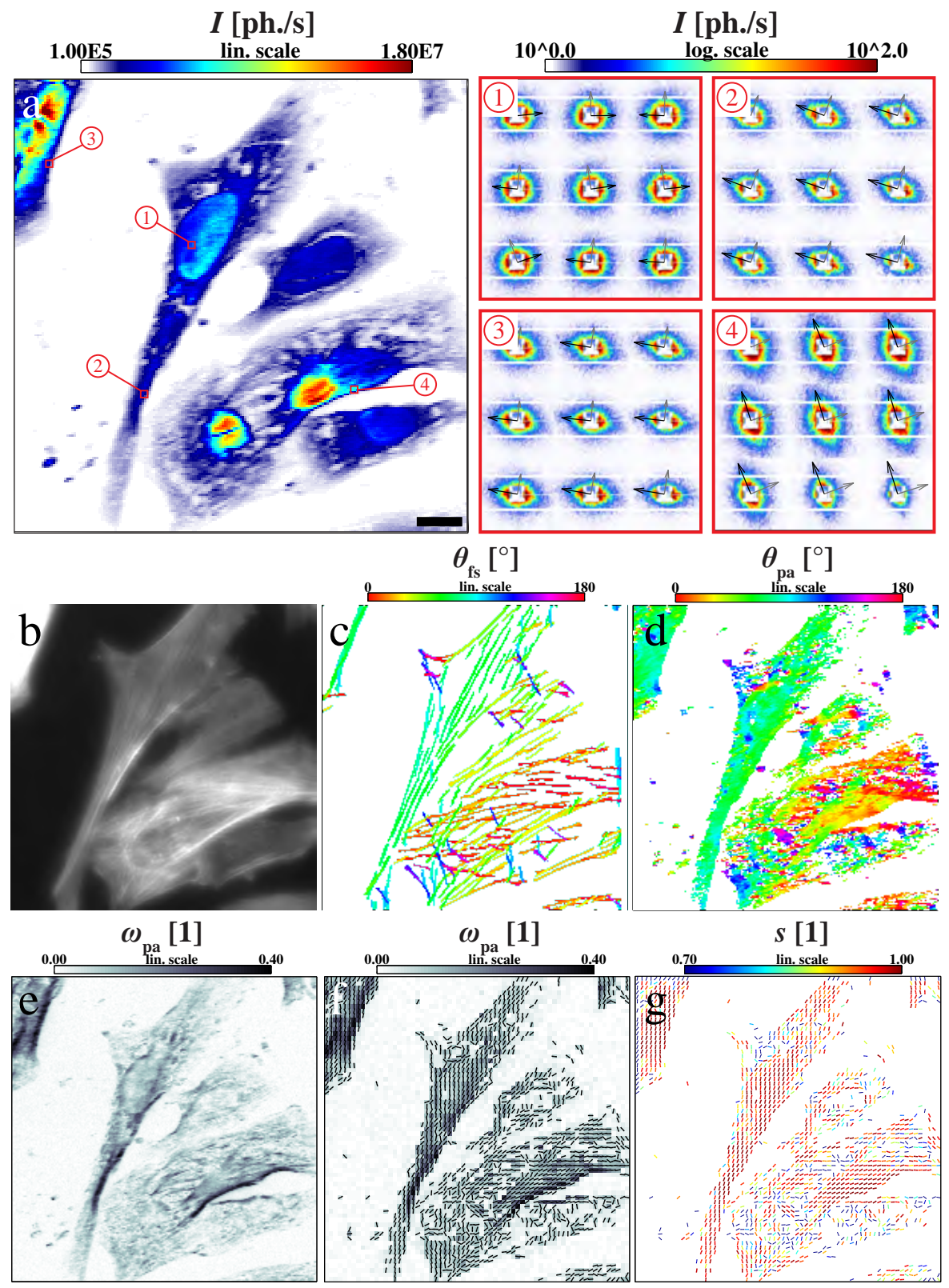

Figure 5.7: (a) left: X-ray dark field of a nano-SAXS scan on freeze-dried NRCTCs. Scale bar: $10 \mu m$. right: Neighboring diffraction patterns of regions (1)-(4) including principal axes $\vec{l}_{k}$ (gray \& black arrows, see also Eq. 3.8. (b) Fluorescence image of the scan area recorded before plunging the sample. (c) Filament orientation $\theta_{\mathrm{fs}}$. (d) $\theta_{\mathrm{pa}}$ results as found by PCA on the $x$-ray diffraction patterns. (e) Results for $\omega_{\mathrm{pa}}$. ( $f$ ) PCA-results of every third diffraction image showing $\omega_{\mathrm{pa}}$ (color code) and $\theta_{\mathrm{pa}}$ thresholded to $\omega_{\mathrm{pa}}>0.04$. (g) $\theta_{\mathrm{pa}}$ (lines) and values for nematic order parameter s evaluated on directly neighboring diffraction patterns. Figure and caption adapted from (43). 


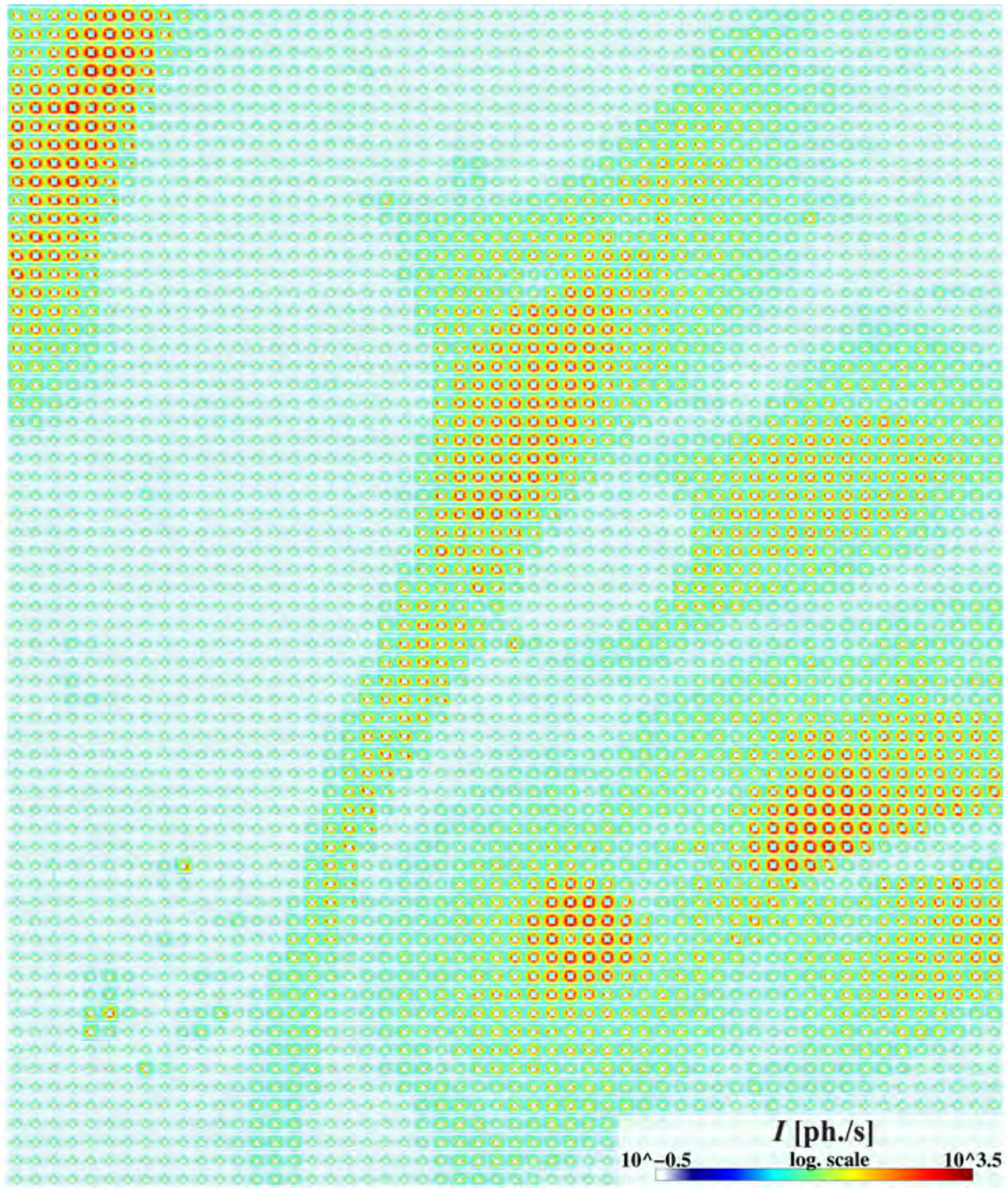

Figure 5.8: Excerpt of the composite image of data presented in Fig. 5.7 showing every third diffraction pattern. Figure and caption adapted from 43.

Fig. 5.7 ( $g$ ) shows the structure orientation angles $\theta_{\text {pa }}$ on every third diffraction image as lines, and the respective value for $s(a, b)$ in color code. $s$ seems to be especially high on regions with relatively pronounced anisotropy (cp. $(g)$ to $(e)$ ) which makes much sense, since esp. here the PCA-algorithm leads to most reliable results. All findings can be reviewed on the composite image of Fig. 5.8 showing every third diffraction pattern. 
Beside these first promising results, data further indicate, that scanning nano-SAXS together with a PCA evaluation of datasets permit us to distinguish between overlaying filamentous cellular structures. This has been proven already on much more pronounced multistreak signals originating from actin in vitro suspensions, see Sec. 3.1.4.

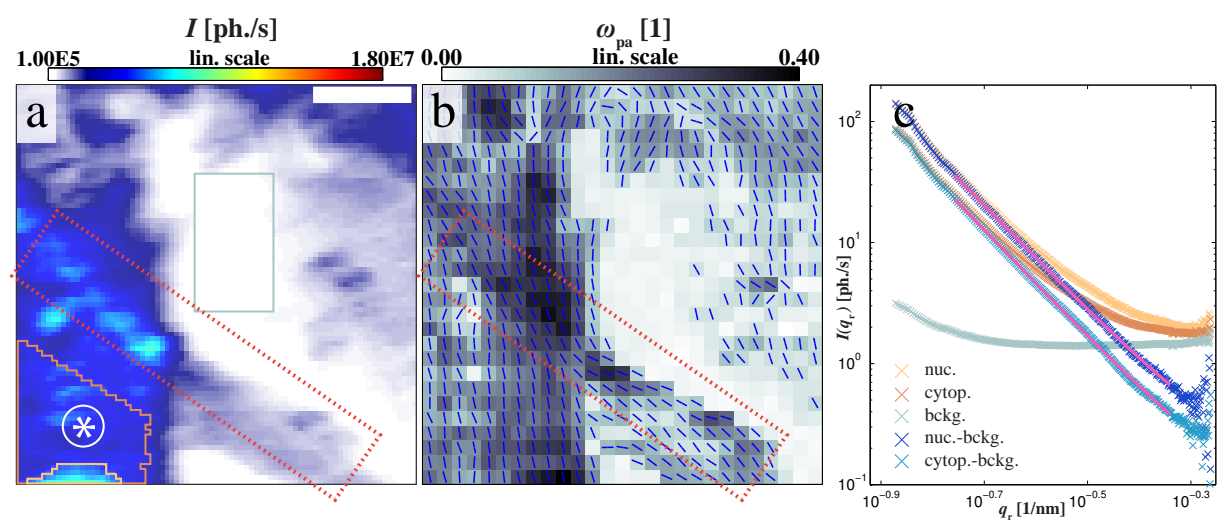

Figure 5.9: (a) X-ray dark field of the cell marked in Fig. 5.10 (b). Scale bar: $5 \mu m$ (b) PCA results showing $\omega_{\mathrm{pa}}{ }^{-}$ values as color code and the structure orientation angles $\theta_{\mathrm{pa}}$ thresholded to $\omega_{\mathrm{pa}}$-values above 0.04 as blue lines. (c) Radial intensity profiles of the regions marked in a including nucleic, cytoplasmic and background signals. The respective deviations are fitted by a (simple) power law function (pink lines, see also Eq.2.5. leading to the results listed in Tab. 5.2

\begin{tabular}{lccc}
\hline & $\mathrm{a}$ & $\mathrm{b}$ & $\mathrm{c}$ \\
\hline nuc.-bckg. & $1.8 \cdot 10^{-2}$ & -4.36 & $1.2 \cdot 10^{-1}$ \\
cytop.-bckg. & $1.0 \cdot 10^{-2}$ & -4.42 & $5.8 \cdot 10^{-2}$ \\
\hline
\end{tabular}

Table 5.2: Results for a (simple) power law fit on the data shown in Fig. 5.9

Fig. 5.9 shows a nano-SAXS scan on a (cryo-protected) freeze-dried sample including the x-ray dark field, see $(a)$, and the PCA-results on every third diffraction patterns, see (b). A single cell labeled by $*$, as identified on a follow-up scan on a larger area (cp. to Fig. 5.10, reveals a preferred filament orientation directing from up to down, see ( $b$; blue lines). This orientation significantly changes when crossing the extension of another cell (see orange dashed rectangle). (c) A further analysis of radial intensity profiles of nucleic, cytoplasmic and background regions of the areas marked in $(a)$ indicate an intensity decay that can be described adequately well by a (simple) power law function (see Eq. 2.5). Results imply an exponent $b<-4$ (see (c), pink lines; results are listed in Tab. 5.2, confirming previous observations of Sec. 2.2.2 


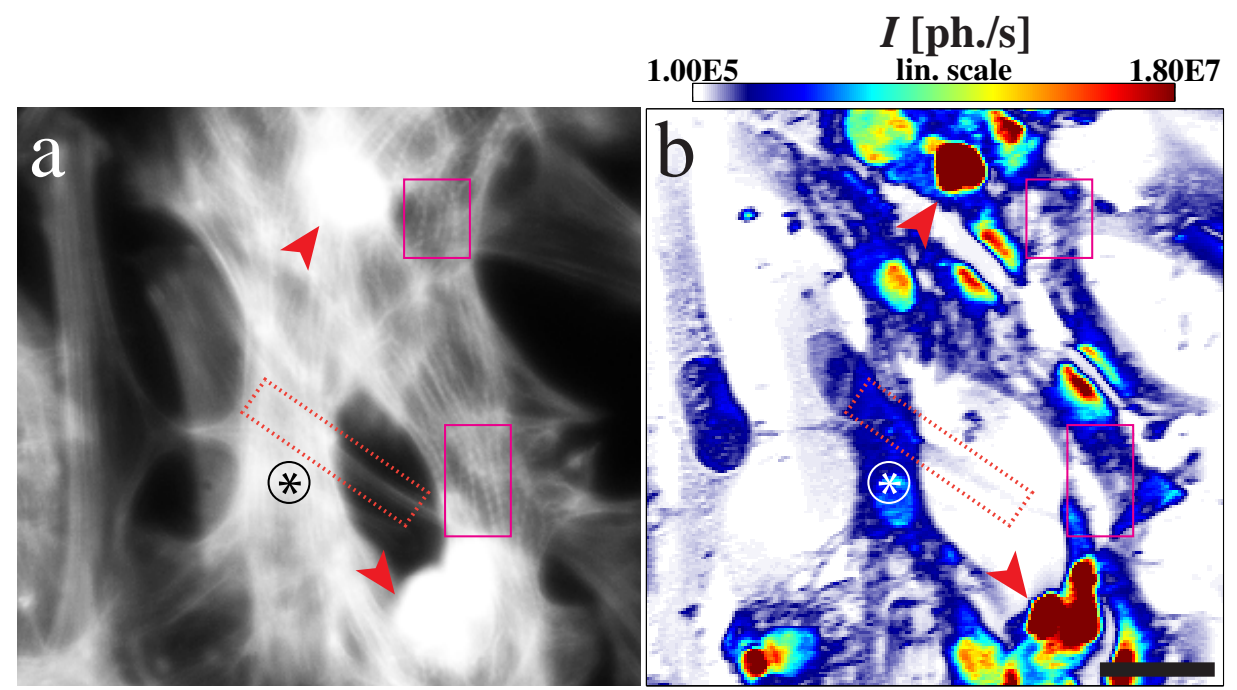

Figure 5.10: (a) Visible light fluorescence image of a sample area before plunging. Regions showing a striation in the fluorescence signals are marked by pink rectangles. Regions of a significantly strong fluorescence signal are marked by red arrows. (b) X-ray dark field of the particular sample area as obtained by a nano-SAXS scan on the freeze-dried sample. Pink rectangles and red arrows correspond to the relative positions marked in (a). * refers to a cell that is investigated in more detail in the previous Fig. 5.9 Scale bar: $20 \mu \mathrm{m}$.

Notably, the focusing capabilities of recent scanning SAXS instruments (such as GINIX) in theory even provide the opportunity to resolve the striated signal of myofibrils in real space, preassuming actin-poor (H Zone of a sarcomere) and actin-rich regions to cause a measurable variation in the overall intensity footprint: Fig. 5.10 (a) shows a broad nano-SAXS scan including the visible light fluorescence image in $(a)$ and the x-ray dark field in $(b)$. Striation in the fluorescence signal indicate the presence of myofibrils at particular spots (see (a), pink rectangles). Comparison of $(a)$ and (b) show that the real space resolution applied here is sufficient. However, we did not observe any striation in a STXM map of any nano-SAXS dataset yet. Fig. 5.10 (b) further reveal two intense maximums in fluorescence and dark field signal (red arrows), which can be attributed to a cell-cell superimposition of an adhered and an unadhered cell.

We are constantly reminded that freeze-dried samples can only be a first intermediate step towards a physiologically more relevant scanning SAXS study. Fig. 5.11 ( $a$ - $b$ ) illustrates two main artifacts associated with freeze-drying by showing (i) significant macroscopic structural defects ( $a$, yellow arrow), here corresponding to a size of $\approx 2 \mu \mathrm{m}$, as well as (ii) questionable cellular dark field intensities, see both $(a)$ and $(b)$.

Preparation of cells in the alive state excludes most of such artifacts and permits ad- 
dressing structural attributes of cells under physiological conditions. First results are presented in the following Sec. 5.4 .
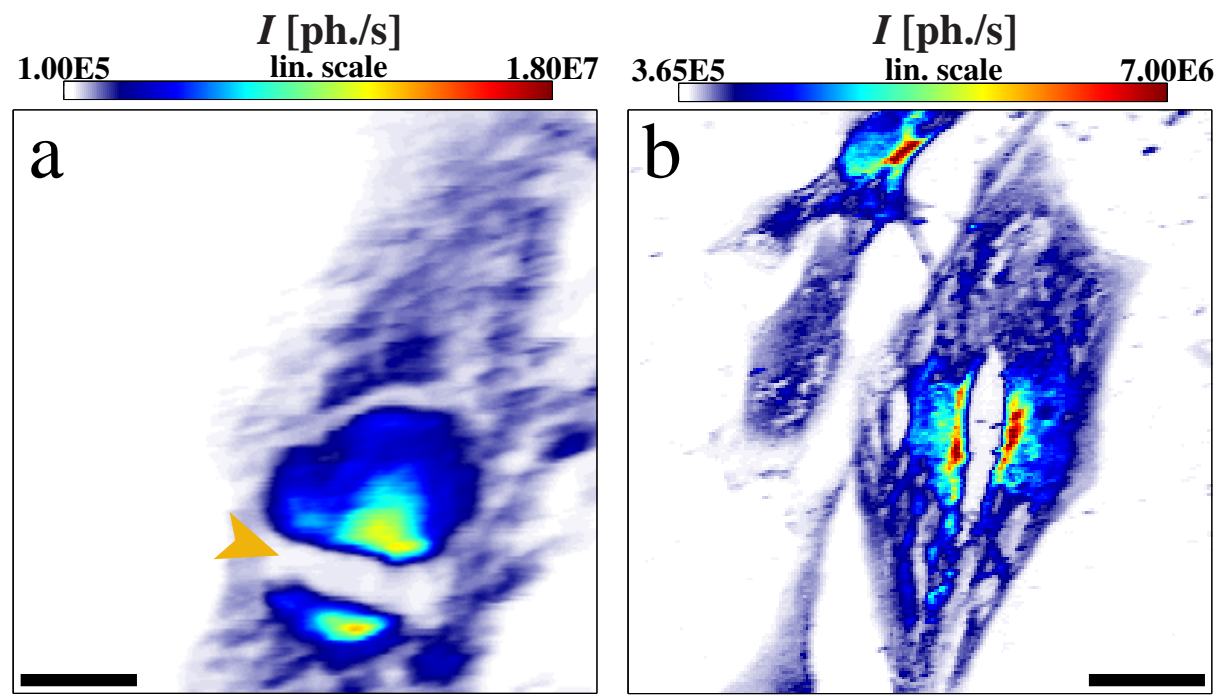

Figure 5.1 1: (a) X-ray dark field of a freeze-dried NRCTC revealing significant disruptions of the nucleus. The gap corresponds to a distance of approx. $2 \mu \mathrm{m}$. (b) X-ray dark field of a freeze-dried sample showing ambiguous overall scattering intensities leading to an inconsistent overall morphology of the cell. Scale bars: $5 \mu m \& 20 \mu m$

\subsection{Alive Recordings}

As a next step towards reliable alive cell recordings, this section pursues the findings of Sec. 4.6 and shows that scanning SAXS on adherent wild-type cells in the alive state are feasible. To this end, commercially available chambers are used comprising two ultrathin silicon nitride windows of $1 \mu \mathrm{m}$ thickness and a spacer of $70 \mu \mathrm{m}$ forming the chamber, see Fig. 5.12 Together with an inner edge length of the spacer measuring $1.5 \mathrm{~mm}$, the chamber contains a total volume of $0.16 \mu \mathrm{L}$. Assuming $\mathrm{H}_{2} \mathrm{O}$ as filling medium, the theoretical transmission is $\mathrm{T}=0.91$ for a photon energy of $E_{\mathrm{ph} .}=8.0 \mathrm{keV}(\underline{62)}$ which, according to the manufacturer, can be further improved by a reduction of the spacer down to $5 \mu \mathrm{m}$ or less 66 .

Fig. 5.13 shows first results of NRCTCs in the alive state. $(a-b)$ X-ray dark fields show overall scattering signals that enable clear identification of the cellular areas. A closer look reveals areas of (rather) high scattering intensities, most presumably denoting the nucleus. (c) PCA results indicate cellular regions with an anisotropic character with 


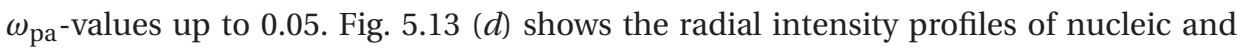
cytoplasmic regions as well as the background signal considering the regions marked in $(b)$. The respective background-corrected deviations are fitted by a (simple) power law function (see Eq. 2.5 leading to exponents $b \approx-3.4$. Further results are listed in Tab. 5.3 aspects of the transfer and mounting procedure of the chamber are further detailed in Appendix Sec. 10.5

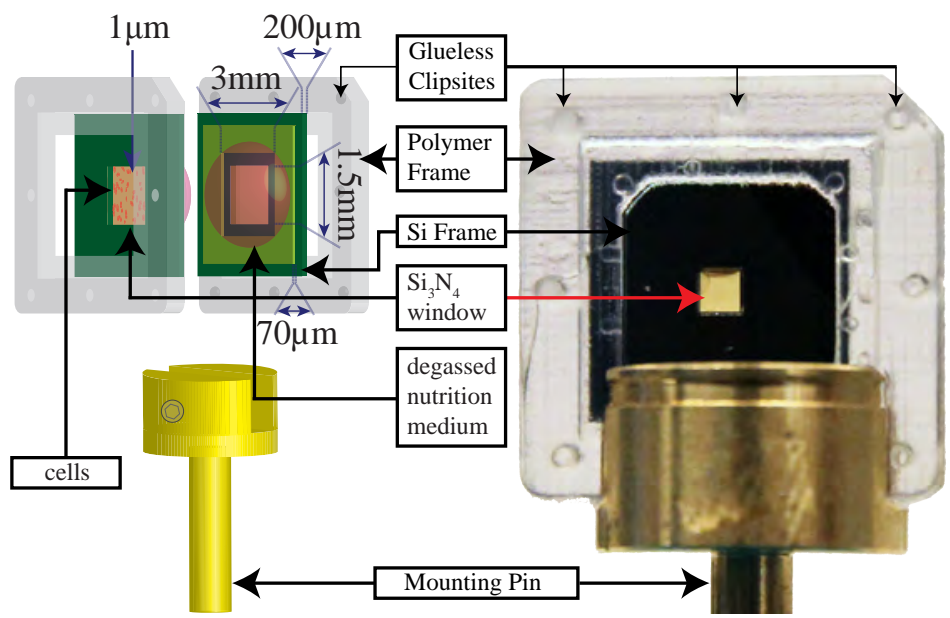

Figure 5.12: (a) Sketch and photograph of a commercially available wet chamber (Silson, UK) on the basis of two ultrathin SiRN-windows, as used for the (initially) alive nano-SAXS experiments. Scans were performed at a photon energy of $E_{\mathrm{ph}}=8 \mathrm{keV}$ and a flux $I_{0}=2.5 \cdot 10^{11} \mathrm{ph} . / \mathrm{s}$. Figure and caption adapted from 43 .

\begin{tabular}{lccc}
\hline & $\mathrm{a}$ & $\mathrm{b}$ & $\mathrm{c}$ \\
\hline nuc.-bckg. & $4.04 e-03$ & -3.38 & $4.28 e-02$ \\
cytop.-bckg. & $1.73 e-03$ & -3.37 & $-1.57 e-03$
\end{tabular}

Table 5.3: Results determined on the background-corrected data plotted in Fig. 5.13 (e) following Eq. 2.5 

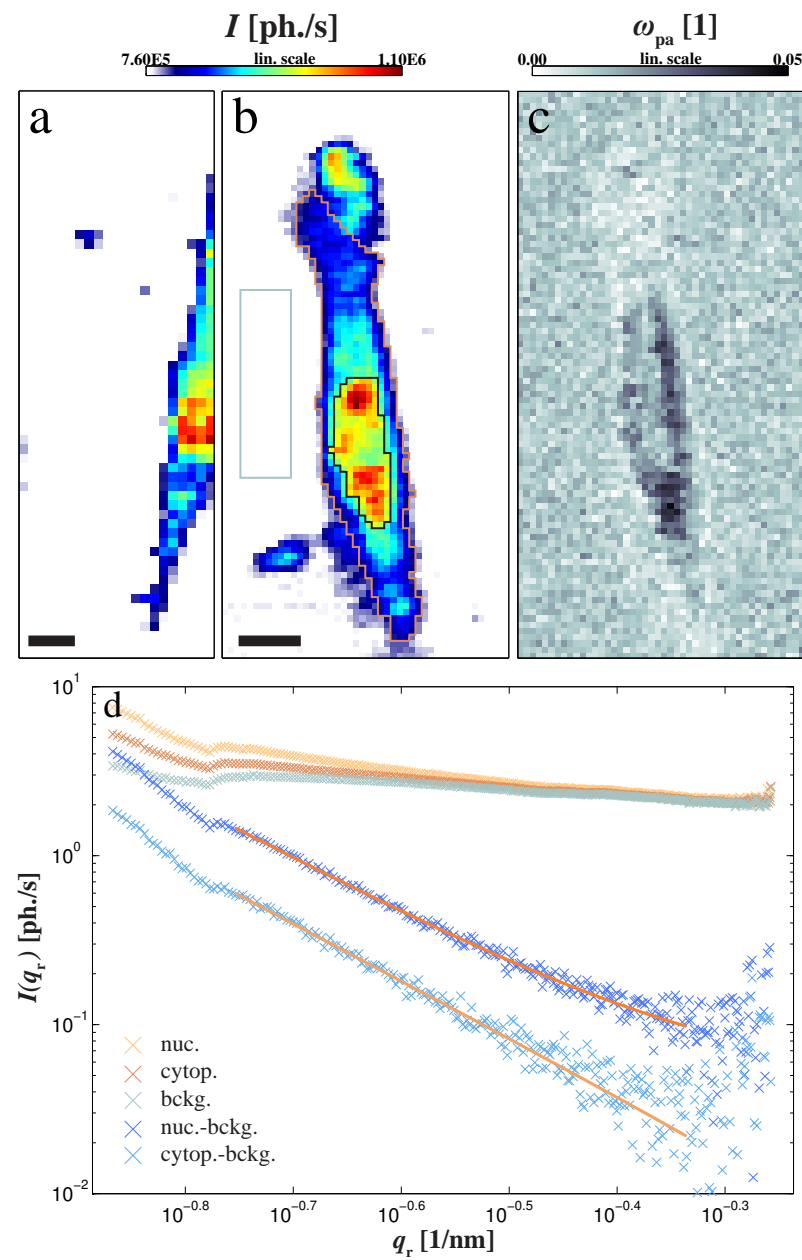

Figure 5.13: $(a-b) X$-ray dark fields of initially alive cells probed by a nano-SAXS beam with step sizes $\Delta=1 \mu \mathrm{m}$ and $0.5 \mu \mathrm{m}$, respectively. Scale bars: $5 \mu \mathrm{m}$. (c) Results for the order parameter $\omega_{\mathrm{pa}}$ as determined by PCA. (d) Averaged radial intensity profiles of nucleic, cytoplasmic and background regions together with the respective background-corrected signals. The latter are fitted by a simple powerlaw decay (pink lines) as defined in Eq. 2.5 leading to the results listed in Tab. 5.3 Figure and caption adapted from 43). 


\section{Section 6}

\section{Summary, Discussion and}

\section{Outlook}

The present work has shown, that structural studies by scanning SAXS on human mesenchymal stem cells and differentiated cell lines, in particular cardiomyocytes, are feasible. The data obtained on freeze-dried samples exhibit an anisotropic signal in their cellular diffraction patterns. This anisotropy is one of the striking features of cellular scanning SAXS and can be attributed to biological protein filaments [27, 31, 42, 63). In this work we have introduced PCA as an appropriate tool to characterize these signals. Analyzing a diffraction pattern by PCA leads to three basic observables for each scan point: The structure orientation angle $\theta_{\mathrm{pa}}$ describing the orientation of the filament and the corresponding two variances $\lambda_{k}$, with $k \in[1,2]$, measuring the second moments of the scattering distribution along both principal axes. The degree of anisotropy can thus be quantified by definition of a unitless order parameter $\omega_{\mathrm{pa}}$ and the corresponding standard deviations $\sigma_{k}=\sqrt{\lambda_{k}}$ are adequate scaling factors for the principal axes in reciprocal space.

When implemented in an automated manner, PCA enables the mapping of entire scan areas. This analysis in principle requires a clean, radial symmetric empty beam profile and an uninterrupted detection area, both of which were available for all micro-SAXS recordings and have thus led to robust results. Notwithstanding, this work has shown, that PCA not only works on our micro-SAXS datasets but also proved to be well-suited for the nano-SAXS datasets presented here with less ideal conditions, leading to a consistent outcome even without any interpolation or post processing of the diffraction 
signals.

When applied to a quantity of scans, PCA together with other automated tools provide the access to statistically robust observables in order to characterize particular aspects of single cells. This work has demonstrated, that a well-established work flow together with a fast recording strategy allows for a statistical investigation of many cells of different cell lines within reasonable synchrotron beam time. The first experiments presented here rely on the micro-SAXS setup, that allows to probe large areas of a sample in a short amount of time by averaging over a large sample area at each scan position (beam size about $50 \mu \mathrm{m}$ ). The results obtained from freeze-dried samples of naive and muscle-induced hMSCs, murine myo- and fibroblasts as well as neonatal rat cardiac tissue cells (NRCTCs) allow to analyze the structural properties of these cell lines on the basis of x-ray dark field and PCA results. To this end, four observables are defined and quantified for each identifiable single cell, comprising the mean scattering intensity $<I>$, the mean anisotropy $\Omega=<\omega_{\text {pa }}>$ as a measure for the overall anisotropic character, the 2D nematic order parameter $s$ as a measure for the consistency among orientation directions as well as the mean standard deviation of the momentum transfer $\langle\bar{\sigma}\rangle$, reflecting the effective sizes of scattering constituents 42 , 43). The data obtained support the hypothesis of naive hMSCs to be rather unstructured cells which acquire structural orientation throughout the differentiation process. Even more importantly, the results emphasize the scope of a scanning SAXS based investigation: Robust, straight forward and model-free algorithms together with high contrast SAXS scans facilitate the characterization of cell type-specific structural attributes on the basis of 2D-diffraction patterns. These patterns encode all local constituents according to their electron density distribution and thus do not rely on any labeling of the specific structures of interest. For this reason, scanning SAXS is very well suited for complementing the results of other structural studies using high resolution imaging techniques such as PALM and STED [35. 39).

However, the critical point of scanning SAXS results is, that diffraction data need to be investigated under specific aspects and interpreted from a biophysical point of view. To this end, several approaches are possible while all of them are equally important: First, an interpretation of data can be addressed by the use of biological model systems such as genetically modified cell lines $[27,-29,63)$ or in vitro samples (32). Second, the recorded diffraction patterns can be compared to simulated data requiring welldefined structural models $31,63,67,70)$. Third, diffraction patterns can be correlated to datasets obtained by other imaging techniques on a particular sample area. This fundamental interplay is also sketched in Fig.6.1. 


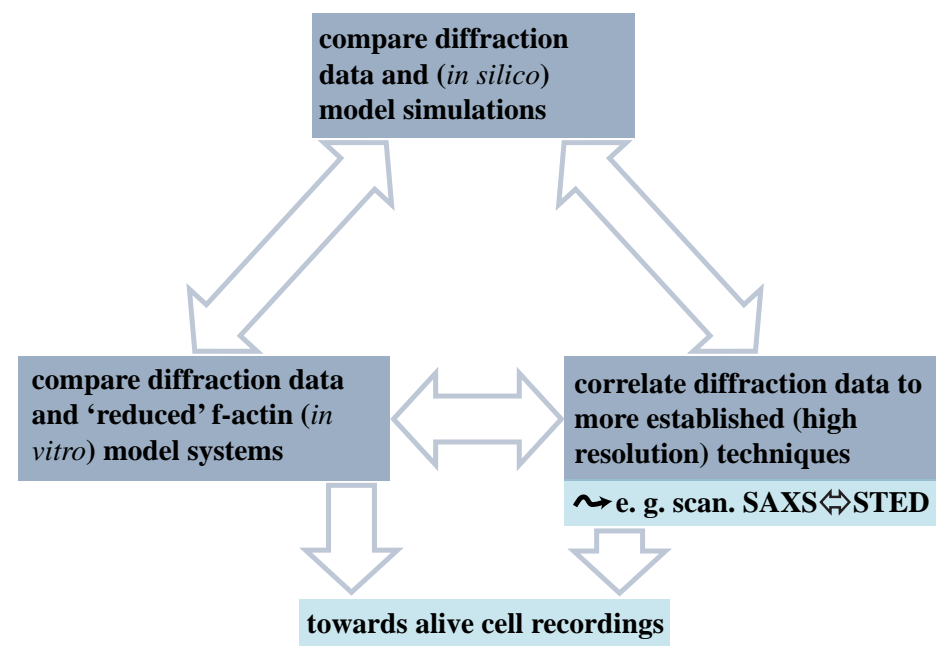

Figure 6.1: Sketch illustrating the three major approaches to interpret cellular scanning SAXS data.

This work has introduced a comparative study on neonatal cardiac tissue cells (NRCTCs) revealing a significant agreement between the filament orientation angles $\theta_{\mathrm{fs}}$ tracked on the visible light fluorescence micrograph and the structure orientation angles $\theta_{\text {pa }}$ as determined by PCA from the x-ray diffraction data. To this end, the fluorescence micrographs of labeled actin are analyzed aiming at the most significant filaments, that are beyond a manually defined threshold. The computed data lead to (yet unbinned) filament orientation angles $\vartheta_{\mathrm{fs}}$ for each pixel of the fluorescence image. Post-processing then leads to adapted results (including the mean filament orientation angle $\theta_{\mathrm{fs}}$ ) that now correspond to the step size chosen for the x-ray scan. This makes possible the correlation of both datasets. Data suggest a strong correlation between the presence of actin filaments and the structure orientation as found on scanning SAXS patterns.

Reducing every diffraction pattern to its overall scattering intensity (x-ray dark field) or its anisotropic character (PCA) is an essential first step in analyzing the cellular dataset. However, this is, for obvious reasons, by far not sufficient to decode all structural properties of a SAXS scan. In general, each SAXS scan contains a tremendous amount of data encoding a wealth of structural information, thus making automated algorithms indispensable tools to characterize the entire scan area. In particular, diffraction patterns comprise local form- and structure factors in their radial intensity profiles as obtained by azimuthal integration of each signal. So far, the data presented here do not reveal any modulations in their radial intensity signals. However, in particular cardiac cell lines show multiple power law decays for low and high- $q$-values, which has also been 
previously reported on other cell lines (29). The present work has proposed a fully empirical model that describes the profiles of entire scan areas in an automated manner. The fitting ansatz is based on a linear combination of $q_{\mathrm{r}}^{p}$ with fixed integer exponents and can be restricted to an interval of $p \in[-8,0]$. All profiles of a scan area can thus be described by coefficients $c^{p}$ of the function leading to a $N_{a} \times N_{b} \times 9$-dimensional array for the scan, with the scan dimensions $N_{a} \times N_{b}$. If further enhanced by other algorithms, the (normalized) $c^{p}$-values enable a direct comparison among the shape of radial intensity profiles at different sample spots or even among different cell lines.

Moreover, this work has shown, that cellular scanning SAXS is feasible in multiple modalities of the x-ray instrument (beam size, photon energy, $q$-range), leading to meaningful and promising results on samples in a freeze-dried, frozen-hydrated, chemically fixated and alive state. When recorded with a highly focused beam (nanoSAXS), samples show x-ray dark field intensities on which one can reliably distinguish between nucleic, cytoplasmic and background regions in all preparation states.

This work has shown, that cryoprotection is capable of reducing radiation damage on freeze-dried samples as caused by the probing beam. To this end, two scan series on cryoprotected freeze-dried murine fibroblasts were performed and evaluated in relation to their x-ray dark field intensities as well as their (background-corrected) average radial intensity decays. Data neither show remarkable alterations in the intensity footprint nor any significant steepening in decays as previously reported for unprotected samples (31). However, while freeze-drying the sample leads to significantly pronounced diffraction signals, it at the same time is associated with an invasive sample preparation procedure causing structural disruptions on the specimen. The mediumterm goal of projects based on cellular scanning SAXS is therefore to obtain nanostructure information on samples under more preserving conditions ultimately leading to quantitative cellular SAXS- or other x-ray based studies on alive cells.

Towards this goal, this work has presented results on (hydrated) chemically fixated or alive cells using multiple (home- or custom-built) chamber geometries. The most promising one is the "self-assembly"-cell (Silson, UK), here used for alive cell recordings, which is also available as a microfluidic chamber version with one (or optionally two) inlet(s) and an outlet.

In conclusion, this work has contributed to further establish scanning SAXS as a powerful tool to complement the repertoire of high resolution imaging techniques by working out preparation, instrumentation and analysis strategies for future projects. 


\section{Section 7}

\section{List of Abbreviations}

\begin{tabular}{ll}
\hline Abbreviation & Meaning \\
\hline a.u. & arbitrary units \\
bckg. & background \\
C2C12 & murine myoblast \\
cp. & compare \\
cSAXS & coherent small-angle x-ray scattering endstation at PSI/SLS \\
cytop. & cytoplasm \\
DESY & Deutsche Elektronen-Synchrotron \\
Dexam. & Dexamethasone \\
df & dark field \\
DPC & differential phase contrast \\
DSMZ & Deutsche Sammulung von Mikroorganismen und Zellkulturen \\
ECM & extracellular matrix \\
Eq. & equation \\
esp. & especially \\
Fig. & figure \\
fs & filament sensor \\
FWHM & full width half maximum \\
GINIX & Göttingen Instrument for Nano-Imaging with X-Rays \\
hMSC & human mesenchymal stem cell \\
Hydroc. & Hydrocortisone \\
KB & Kirkpatrick-Baez \\
\hline
\end{tabular}




\begin{tabular}{ll}
\hline Abbreviation & Meaning \\
\hline lin. scale & linear scale \\
LN2 & liquid nitrogen \\
log. scale & logarithmic scale \\
micro-SAXS & microfocus SAXS \\
$\mathbb{N}$ & natural numbers \\
nano-SAXS & nanofocus SAXS \\
NIH-3T3 & murine fibroblast \\
NRCTC & neonatal rat cardiac tissue cell \\
nuc. & nucleus \\
OAV & on-axis video microscope \\
pa & principal axis \\
PCA & principal component analysis \\
pen. & Penicillin \\
PETRA & Positron-Elektron-Tandem-Ring-Anlage \\
ph. & photons \\
PSI & Paul-Scherrer Institute \\
px & pixel \\
$\mathbb{R}$ & real numbers \\
ROI & region of interest \\
SAXS & small angle x-ray scattering \\
Sec. & section \\
SLS & Swiss Light Source \\
SiRN & silicon-rich nitride \\
Strep. & Streptomycin \\
STXM & scanning transmission x-ray microscopy \\
Tab. & table \\
tr & transmission \\
$\mathbb{Z}$ & integer numbers \\
\hline
\end{tabular}




\section{Section 8}

\section{Bibliography}

1. Bruce Alberts, Alexander Johnson, Julian Lewis, Martin Raff, Keith Roberts, and Peter Walter. Molecular Biology of the Cell. Garland Science, 2008, Fifth edition.

2. Patricia A. Zuk, Min Zhu, Peter Ashjian, Daniel A. De Ugarte, Jerry I. Huang, Hiroshi Mizuno, Zeni C. Alfonso, John K. Fraser, Prosper Benhaim, and Marc H. Hedrick. Human adipose tissue is a source of multipotent stem cells. Molecular Biology of the Cell, 2002.

3. Adam J. Engler, Shamik Sen, H. Lee Sweeney, and Dennis E. Discher. Matrix elasticity directs stem cell lineage specification. Cell, 2006.

4. Nora G. Singer and Arnold I. Caplan. Mesenchymal stem cells: Mechanisms of inflammation. Annu. Rev. Pathol. Mech. Dis., 2011.

5. S. Deb, K. Ghosh, and S. D. Shetty. Nanoimaging in cardiovascular diseases: Current state of the art. Indian J Med Res, 141:285-298, 2015.

6. S. Makino, K. Fukuda, S. Miyoshi, F. Konishi, H. Kodama, J. Pan, M. Sano, T. Takahashi, S. Hori, H. Abe, J. Hata, A. Umezawa, and S. Ogawa. Cardiomyocytes can be generated from marrow stromal cells in vitro. The Journal of Clinical Investigation, 103:697-705, 1999.

7. C. Toma, M. F. Pittenger, K. S. Cahill, B. J. Byrne, and P. D. Kessler. Human mesenchymal stem cells differentiate to a cardiomyocyte phenotype in the adult murine heart. Circulation, 105:93-98, 2002. 
8. Sunil Rangappa, John W.C Entwistle, Andrew S Wechsler, and J.Yasha Kresh. Cardiomyocyte-mediated contact programs human mesenchymal stem cells to express cardiogenic phenotype. The Journal of Thoracic and Cardiovascular Surgery, 126(1):124 - 132, 2003.

9. S. Tojkander, G. Gateva, and P. Lappalainen. Actin stress fibers-assembly, dynamics and biological roles. J. Cell. Sci., 125(Pt 8):1855-1864, Apr 2012.

10. A. Zemel, F. Rehfeldt, A. E. X. Brown, D. E. Discher, and S. A. Safran. Optimal matrix rigidity for stress-fibre polarization in stem cells. nature physics, 2010.

11. Marina Eckermann. Scanning small-angle x-ray diffractive imaging of cardiomyocyte cells. Bachelor Thesis, 2014.

12. H. E. Huxley. Fifty years of muscle and the sliding filament hypothesis. Eur. J. Biochem., 271:1403-1415, 2004.

13. K. Jefimovs, O. Bunk, F. Pfeiffer, D. Grolimund, J.F. van der Veen, and C. David. Fabrication of fresnel zone plates for hard x-rays. Microelectronic Engineering, 84:14671470, 2007.

14. A. Snigirev, V. Kohn, I. Snigireva, and B. Lengeler. A compound refractive lens for focusing high-energy x-rays. Nature, 384:49-51, 1996.

15. C. G. Schroer, M. Kuhlmann, U. T. Hunger, T. F. Günzler, O. Kurapova, S. Feste, F. Frehse, B. Lengeler, M. Drakopoulos, A. Somogyi, A. S. Simionovici, A. Snigirev, I. Snigireva, C. Schug, and W. H. Schröder. Nanofocusing parabolic refractive X-ray lenses. Appl. Phys. Let., 82, No. 9:1485-1487, 2003.

16. C. G. Schroer, O. Kurapova, J. Patommel, P. Boye, J. Feldkamp, B. Lengeler, M. Burghammer, C. Riekel, L. Vincze, A. van der Hart, and M. Küchler. Hard X-ray nanoprobe based on refractive x-ray lenses. Appl. Phys. Let., 87:124103-1-124103$3,2005$.

17. O. Hignette, P. Cloetens, G. Rostaing, P. Bernard, and C. Morawe. Efficient sub 100 $\mathrm{nm}$ focusing of hard x rays. Review of Scientific Instruments, 76(6):063709, 2005.

18. H. Mimura, H. Yumoto, S. Matsuyama, Y. Sano, K. Yamamura, Y. Mori, M. Yabashi, Y. Nishino, K. Tamasaku, T. Ishikawa, and K. Yamauchi. Efficient focusing of hard x rays to $25 \mathrm{~nm}$ by a total reflection mirror. Applied Physics Letters, 90:051903-1051903-3, 2007. 
19. H. Mimura, S. Handa, T. Kimura, H. Yumoto, D. Yamakawa, H. Yokoyama, S. Matsuyama, K. Inagaki, K. Yamamura, Y. Sano, K. Tamasaku, Y. Nishino, M. Yabashi, T. Ishikawa, and K. Yamauchi. Breaking the 10nm barrier in hard-X-ray focusing. Nature Physics, 6:122-125, 2010.

20. Tim Salditt, Markus Osterhoff, Martin Krenkel, Robin N. Wilke, Marius Priebe, Matthias Bartels, Sebastian Kalbfleisch, and Michael Sprung. Compound focusing mirror and X-ray waveguide optics for coherent imaging and nano-diffraction. J. Synchrotron Rad., 22(4):867-878, Jul 2015.

21. F. Döring, A. L. Robisch, C. Eberl, M. Osterhoff, A. Ruhlandt, T. Liese, F. Schlenkrich, S. Hoffmann, M. Bartels, T. Salditt, and H. U. Krebs. Sub-5 nm hard x-ray point focusing by a combined kirkpatrick-baez mirror and multilayer zone plate. Optics Express, 21, No. 16:19311-19323, 2013.

22. S. Kalbfleisch, M. Osterhoff, K. Giewekemeyer, H. Neubauer, S.P. Krüger, B. Hartmann, M. Bartels, M. Sprung, O. Leupold, F. Siewert, and T. Salditt. The holography endstation of beamline p10 at petra iii. AIP. Conf. Proc., 1234:433-436, 2010. Beamline P10 endstation GINIX.

23. S. Kalbfleisch, H. Neubauer, S. P. Krüger, M. Bartels, M. Osterhoff, D. D. Mai, K. Giewekemeyer, B. Hartmann, M. Sprung, and T. Salditt. The göttingen holography endstation of beamline p10 at petra iii/desy. AIP Conference Proceedings, 1365(1):96-99, 2011. Beamline P10 endstation GINIX.

24. Sebastian Kalbfleisch. A Dedicated Endstation for Waveguide-based X-Ray Imaging. PhD thesis, 2012.

25. Heimo Schnablegger and Yashveer Singh. The SAXS guide (getting acquainted with the principles). Paar, Anton, 3rd edition.

26. Jens Als-Nielsen and Des McMorrow. Elements of Modern X-ray Physics. Wiley, 2011.

27. B. Weinhausen, J.-F. Nolting, C. Olendrowitz, J. Langfahl-Klabes, M. Reynolds, T. Salditt, and S. Köster. X-ray nano-diffraction on cytoskeletal networks. New J. Phys., 14(8):085013, 2012.

28. Britta Weinhausen. Scanning X-Ray Nano-Diffraction on Eukaryotic Cells: From Freeze-Dried to Living Cells. PhD thesis, Göttingen, 2013. 
29. B. Weinhausen, O. Saldanha, R. N. Wilke, C. Dammann, M. Priebe, M. Burghammer, M. Sprung, and S. Köster. Scanning x-ray nanodiffraction on living eukaryotic cells in microfluidic environments. Phys. Rev. Lett., 112(8):088102, February 2014.

30. R. N. Wilke, M. Priebe, M. Bartels, K. Giewekemeyer, A. Diaz, P. Karvinen, and T. Salditt. Hard x-ray imaging of bacterial cells: nano-diffraction and ptychographic reconstruction. Opt. Express, 20(17):19232-19254, August 2012.

31. M. Priebe, M. Bernhardt, C. Blum, M. Tarantola, E. Bodenschatz, and T. Salditt. Scanning X-ray nanodiffraction on dictyostelium discoideum. Biophysical Journal, 107(11):2662 - 2673, 2014.

32. M. Töpperwien, M. Priebe, and T. Salditt. Actin bundles cross-linked with alphaactinin studied by nanobeam x-ray diffraction. European Biophysics Journal, pages $1-10,2015$.

33. Joseph Strzalka. Cellular diffraction: Scanning x-ray nanodiffraction from living cells. Biophysical Journal, 107:2489, 2014.

34. R. N. Wilke, M. Hoppert, M. Krenkel, M. Bartels, and T. Salditt. Quantitative x-ray phase contrast waveguide imaging of bacterial endospores. J. Appl. Crystallogr., 48(2):464-476, Apr 2015.

35. T. A. Klar, S. Jakobs, M. Dyba, A. Egner, and S. W. Hell. Fluorescence microscopy with diffraction resolution barrier broken by stimulated emission. Proc. Natl. Acad. Sci. U.S.A., 97(15):8206-8210, Jul 2000.

36. V. Westphal and S. W. Hell. Nanoscale resolution in the focal plane of an optical microscope. Phys. Rev. Lett., 94(14):143903, Apr 2005.

37. S. T. Hess, T. P. Girirajan, and M. D. Mason. Ultra-high resolution imaging by fluorescence photoactivation localization microscopy. Biophys. J., 91(11):4258-4272, Dec 2006.

38. E. Betzig, G. H. Patterson, R. Sougrat, O. W. Lindwasser, S. Olenych, J. S. Bonifacino, M. W. Davidson, J. Lippincott-Schwartz, and H. F. . Imaging intracellular fluorescent proteins at nanometer resolution. Science, 313(5793):1642-1645, Sep 2006.

39. M. J. Rust, M. Bates, and X. Zhuang. Sub-diffraction-limit imaging by stochastic optical reconstruction microscopy (STORM). Nat. Methods, 3(10):793-795, Oct 2006.

40. H. E. Huxley and W. Brown. Low-angle x-ray diagram. of vertebrate striated muscle and its behaviour during contraction and rigor. J. Mol. BioI., 30:383-434, 1967. 
41. H. E. Huxley. A personal view of muscle and motility mechanisms. Annu Rev. Physiol., 58:1-19, 1996.

42. M. Bernhardt, M. Priebe, M. Osterhoff, C. Wollnik, A. Diaz, T. Salditt, and F. Rehfeldt. X-Ray Micro- and Nanodiffraction Imaging on Human Mesenchymal Stem Cells and Differentiated Cells. Biophys. J., 110(3):680-690, Feb 2016.

43. Marten Bernhardt, Jan-David Nicolas, Marina Eckermann, Benjamin Eltzner, Florian Rehfeldt, and Tim Salditt. Anisotropic x-ray scattering and orientation fields in cardiac tissue cells. New Journal of Physics, 19(1):013012, 2017.

44. Y. Takahashi, A. Suzuki, N. Zettsu, T. Oroguchi, Y. Takayama, Y. Sekiguchi, A. Kobayashi, M. Yamamoto, and M. Nakasako. Coherent diffraction imaging analysis of shape-controlled nanoparticles with focused hard x-ray free-electron laser pulses. Nano Lett., 13(12):6028-6032, November 2013.

45. H. Lichtenegger, M. Müller, O. Paris, Ch. Riekel, and P. Fratzl. Imaging of the helical arrangement of cellulose fibrils in wood by synchrotron X-ray microdiffraction. Journal of Applied Crystallography, 32(6):1127-1133, Dec 1999.

46. O. Sumner Makin, Pawel Sikorski, and Louise C. Serpell. CLEARER: a new tool for the analysis of X-ray fibre diffraction patterns and diffraction simulation from atomic structural models. Journal of Applied Crystallography, 40(50):966-972, Oct 2007.

47. O. Bunk, M. Bech, T. H. Jensen, R. Feidenhans'l, T. Binderup, A. Menzel, and F. Pfeiffer. Multimodal x-ray scatter imaging. New Journal of Physics, 11(12):123016, 2009.

48. K Pearson. On lines and planes of closest fit to systems of points in space. Philosophical Magazine, 2:559-572, 1901.

49. J. Edward Jackson. A Users Guide To Principal Components. Wiley, 1991.

50. B. Henrich, A. Bergamaschi, C. Broennimann, R. Dinapoli, E.F. Eikenberry, I. Johnson, M. Kobas, P. Kraft, A. Mozzanica, and B. Schmitt. Pilatus: A single photon counting pixel detector for x-ray applications. Nuclear Instruments and Methods in Physics Research Section A: Accelerators, Spectrometers, Detectors and Associated Equipment, 607(1):247 - 249, 2009.

51. B. Eltzner, C. Wollnik, C. Gottschlich, S. Huckemann, and F. Rehfeldt. The filament sensor for near real-time detection of cytoskeletal fiber structures. PLOS ONE, 10(5):e0126346, 052015. 
52. T. Hotz and S. Huckemann. Intrinsic means on the circle: Uniqueness, locus and asymptotics. arXiv:1108.2141, Aug 2011.

53. https://ece.uwaterloo.ca/ dwharder/NumericalAnalysis/06LeastSquares/general/. last accessed on 17/02/16.

54. http://de.mathworks.com/help/curvefit/least-squares fitting.html. last accessed on $17 / 02 / 16$.

55. B. Liu, T. H. Besseling, M. Hermes, A. F. Demirors, A. Imhof, and A. van Blaaderen. Switching plastic crystals of colloidal rods with electric fields. Nat Commun, 5:3092, 2014 .

56. Florian Rehfeldt, Andre E. X. Brown, a Allison L. Zajac Assaf Zemelc Matthew Raab, a Shenshen Cai, and Dennis E. Discher. Hyaluronic acid matrices show matrix stiffness in $2 \mathrm{~d}$ and $3 \mathrm{~d}$ dictates cytoskeletal order and myosin-ii phosphorylation within stem cellswz. Integrative Biology, 2012.

57. E. K. Paluch, C. M. Nelson, N. Biais, B. Fabry, J. Moeller, B. L. Pruitt, C. Wollnik, G. Kudryasheva, F. Rehfeldt, and W. Federle. Mechanotransduction: use the force(s). BMC Biol., 13:47, 2015.

58. Steve P. Meisburger, Matthew Warkentin, Huimin Chen, Jesse B. Hopkins, Richard E. Gillilan, Lois Pollack, and Robert E. Thorne. Breaking the radiation damage limit with cryo-saxs. Biophysical Journal, 104:227236, January 2013. Cryoprotection.

59. Jan-David Nicolas, Marten Bernhardt, Martin Krenkel, Stefan Luther, and Tim Salditt. Combined x-ray nano-diffraction and holographic imaging of cardiomyocytes. unpublished work (submitted).

60. M. R. Howells, T. Beetz, H. N. Chapman, C. Cui, J. M. Holton, C. J. Jacobsen, J. Kirz, E. Lima, S. Marchesini, H. Miao, D. Sayre, D. A. Shapiro, J. C. Spence, and D. Starodub. An assessment of the resolution limitation due to radiation-damage in $\mathrm{x}$-ray diffraction microscopy. J Electron Spectros Relat Phenomena, 170(1-3):4-12, Mar 2009 .

61. Marius Priebe. Scanning X-Ray Nanodiffraction on Dictyostelium discoideum. $\mathrm{PhD}$ thesis, 2015.

62. http://www.cxro.lbl.gov/. 
63. C. Y. Hemonnot, M. Mauermann, H. Herrmann, and S. Koster. Assembly of Simple Epithelial Keratin Filaments: Deciphering the Ion Dependence in Filament Organization. Biomacromolecules, 16(10):3313-3321, Oct 2015.

64. K. Luby-Phelps. Cytoarchitecture and physical properties of cytoplasm: Volume, viscosity, diffusion, intracellular surface area. International Review o Cytology, 192:189-221, 2000.

65. D. Bray and C. Thomas. The actin content of fibroblasts. Biochem. J., 147:221-228, 1975.

66. http://www.silson.com.

67. P. Szekely, A. Ginsburg, T. Ben-Nun, and U. Raviv. Solution X-ray scattering form factors of supramolecular self-assembled structures. Langmuir, 26(16):1311013129, Aug 2010.

68. M. E. Brennich, S. Bauch, U. Vainio, T. Wedig, H. Herrmann, and S. Köster. Impact of ion valency on the assembly of vimentin studied by quantitative small angle Xray scattering. Soft Matter, 10(12):2059-2068, Mar 2014.

69. J. M. Squire, H. A. Al-Khayat, and Naoto Yagi. Muscle thin-filament structure and regulation. J. Chem. Soc. Faraday Trans., 89 No.15:2717-2726, 1993.

70. H. A. Al-Khayat, N. Yagi, and J. M. Squire. Structural changes in actin-tropomyosin during muscle regulation: computer modelling of low-angle X-ray diffraction data. J. Mol. Biol., 252(5):611-632, Oct 1995.

71. http://www.leica-microsystems.com/de/produkte/emprobenvorbereitung/kryo-praeparations-systeme/details/product/leica-em-gp $1 /$.

72. www.vclub forum.de.

73. https://de.wikipedia.org/wiki/ICE_3. 


\section{Section 9}

\section{Danksagung}

Mein herzlicher Dank gilt zu allererst meinem Hauptbetreuer Prof. Dr. Tim Salditt für die Möglichkeit, eine Doktorarbeit im Institut für Röntgenphysik anfertigen zu können. Ich danke ihm sehr für seine kontinuierliche fachliche Betreuung, seine Chancen eröffnende Beratung der letzten vier Jahre, für seinen Rückhalt sowie die stets kollegiale Arbeitsatmosphäre.

Weiterhin möchte ich mich bei meinem Co-Betreuer Dr. Florian Rehfeldt für die vielen fachlichen Anregungen, die begleitenden Gesprächen, seinen unermüdlichen zeitlichen Einsatz während meines Aufenthaltes sowie für sein Engagement bei diversen Messzeiten in Hamburg und in der Schweiz herzlich bedanken. In meinen Dank schließe ich das gesamte Dritte Physikalische Institut um Prof. Dr. Schmidt für die Bereitstellung der Laborräumlichkeiten zur Zellpräparation ein.

Mein Dank gilt weiterhin Prof. Dr. Sarah Köster und Dr. Britta Weinhausen für die Unterstützung bei der Probenpräparation und der Koordination von Messzeiten, dem Erfahrungsaustausch, den vielen fachlichen Gesprächen sowie der unkomplizierten Unterstützung bei Lehraufträgen.

Mein besonderer Dank gilt meinen derzeitigen und ehemaligen Kollegen Dr. Marius Priebe, Dr. Robin Wilke, Dr. Markus Osterhoff, Dr. Martin Krenkel, Mareike Töpperwien und Yihui Xu für die unzähligen fachlichen und persönlichen Gespräche und ihre tatkräftige Unterstützung bei Messzeiten. Insbesondere genannt sein soll die Unterstützung von Dr. Marius Priebe bei der cryogenen Probenpräparation, seiner Unterstützung bei Messzeiten, seiner Lesekorrektur meiner Manuskripte und ganz allgemein seinem Engagement. 
Ebenfalls möchte ich mich besonders für die tatkräftige Hilfe von Dr. Markus Osterhoff bei der Micro-SAXS Messzeit sowie für seine Lesekorrektur bedanken. Dr. Robin Wilke gebührt mein Dank für die engagierte Einführung in den Lehrversuch "optische Pinzette".

Des Weiteren möchte ich mich für eine gute Zusammenarbeit mit Jan-David Nicolas bezüglich des Kardiomyozytenprojektes, Dr. Benjamin Eltzner für seine zusätzliche Arbeit an einer angepassten Version des Filament Sensors und Johannes Hagemann für weiterführende Vorschläge bei der Datenauswertung bedanken.

Besonders hervorheben möchte ich die Hilfe von Carina Wollnik für ihre Unterstützung bei der biologischen Probenpräparation. Ich danke Dr. Anna-Lena Robisch und Sarah Hoffmann für ihre Diskussionsanregungen und Marina Eckermann für die zusätzlichen Resultate im Rahmen einer Bachelorarbeit.

Ich bedanke mich bei Mike Kanbach für die Präparation von Deckgläsern für die Glaskammer, Bastian Hartmann für die Unterstützung bei Vorbereitungen von GINIXMesszeiten und Jochen Herbst für viele laborrelevante Tipps.

Dr. Ana Diaz danke ich für die Betreuung bei der Micro-SAXS Messzeit und Dr. Michael Sprung für die Unterstützung bei mehreren Nano-SAXS Messzeiten. Mein Dank gilt auch Prof. Dr. Stefan Luther, Dr. Marco Tarantola, Tina Althaus und Marion Kunze für die Bereitstellung neonatalen Rattenkardiomyocyten.

Unserem Administator Jan Goemann danke ich herzlich für seine engagierte Unterstützung hinsichtlich der Infrastuktur des Institutsnetzwerkes, ohne die eine zeitnahe und sichere Datenauswertung nicht möglich gewesen wäre. Des Weiteren möchte ich mich bei unseren derzeitigen und ehemaligen technischen Assistenten Julia Scherber, Kristin Müller und insbesondere Susanne Hengst für ihre engagierte Laborarbeit bedanken, sowie bei Peter Nieschalk aus der Feinmechanik für seine individuellen Anpassungen von Probensystemen.

Mein Dank gilt ebenfalls Prof. Dr. Andreas Janshoff für seine Teilnahme an dem Projekt als drittes Thesis-Committee-Mitglied und Prof. Dr. Ulrich Palitz, Dr. Alexander Egner und Dr. Dieter Klopfenstein für die Teilnahme an meinem Prüfungskomitee.

Last but not least danke ich meinen Eltern herzlich für ihre nicht nur finanzielle Unterstützung, ihr Verständnis, ihre Geduld und ihre Ermutigungen sowie meinen langjährigen Freunden, die mich begleitend unterstützt haben.

Diese Arbeit wurde finanziell unterstützt von der Deutschen Forschungsgemeinschaft mit dem Sonderforschungsbereich SFB 755 "Nanoscale Photonic Imaging" in den Projekten C01 und B08, dem Sonderforschungsbereich SFB 937 "Collective Behavior of Soft and Biological Matter" in dem Projekt A11, dem Virtual Institute No. 
VH-VI-403 "In Situ Nano Imaging of Biological and Chemical Processes" (Helmholtz Society) sowie der Volkswagen Stiftung im Rahmen des Niedersächsisch-Israelischen Netzwerks (Zuschuss-Nummer: MWK-VWZN2722). 


\section{Section 10}

\section{Appendix}

\subsection{X-Ray Instrumentation (Detailed)}

This section is taken from (42).

\section{Instrumentation}

\section{Nano-focus setup}

Nano-SAXS data were recorded at the P10-beamline of DESY's PETRAIII storage ring, using the Göttingen Instrument for Nano-Imaging with X-rays (GINIX) (23). Freezedried and chemically fixed samples in glass chambers were recorded at a photon energy of $E_{\text {ph. }}=7.9 \mathrm{keV}$ (P10/1 ${ }^{\text {st }}$ run). The beam was focused by a pair of KirkpatrickBaez $(\mathrm{KB})$ mirrors to a size of $\approx 250 \times 320 \mathrm{~nm}$ (vert. $\times$ horz., FWHM) as measured by translation of a waveguide. Two soft-edge apertures were placed in front of the sample in order to block parasitic x-rays from the mirrors. The focused photon flux measured at the detector was $I_{0}=1.29 \cdot 10^{11} \mathrm{ph}$./s. The beam could be attenuated using a set of aluminum foils. The sample was mounted on a motorized stage and placed in the focus at about $\approx 20 \mathrm{~cm}$ downstream from the KB-mirrors. An on-axis video microscope $(\mathrm{OAV})$ with 10-30× optical zoom, a working distance of $50 \mathrm{~mm}$, and LED illumination was used for alignment and to define suitable scan regions. Optionally, cryogenic conditions could be applied to the sample via a cryogenic nitrogen gas stream (Oxford instruments, UK). The diffraction patterns for each scan point were recorded by the 
single photon counting pixel detector Pilatus 300k (Dectris, Switzerland), positioned $\approx 5.1 \mathrm{~m}$ behind the focal plane. To reduce absorption, the beam was guided through an evacuated tube. The primary beam was blocked by multiple semi-transparent beam stops. Scans were performed in a discrete or continuous mode with a step size of typically $\Delta_{\text {real }}=0.5-1 \mu \mathrm{m}$, defining the real space resolution. In the discrete scanning mode, the beam was blocked by a fast shutter when moving the sample, while in continuous mode the beam shutter remained open when scanning along the fast axis, i.e. along the y-direction. Recordings of hydrated cells in commercial cell culture chambers were performed at $E_{\mathrm{ph} .}=13.8 \mathrm{keV}\left(\mathrm{P} 10 / 2^{\text {nd }} \mathrm{run}\right)$ with a maximum flux of $I_{0}=1.38 \cdot 10^{11} \mathrm{ph}$. $/ \mathrm{s}$ measured at the detector and a focus size of $\approx 180 \times 370 \mathrm{~nm}$ (vert. $\times$ horz., FWHM) as measured by translation of a waveguide. A set of molybdenum foils was used for attenuation, all other parameters were comparable to the $\mathrm{P} 10 / 1^{\text {st }}$ run.

\section{Micro-focus setup}

Micro-SAXS data were recorded at the cSAXS-beamline of the Swiss Light Source. The X-ray beam was focused by a $\mathrm{Si}(111)$ crystal monochromator in the horizontal and a Rh-coated mirror in the vertical direction resulting in a photon energy of $E_{\mathrm{ph} .}=8.7 \mathrm{keV}$ and a beam size of about $33 \mu \mathrm{m} \times 54 \mu \mathrm{m}$ (vert. $\times$ horz., FWHM) at the sample position. The primary flux measured at the detector was $I_{0}=1.44 \cdot 10^{11} \mathrm{ph} . / \mathrm{s}$. The sample was positioned in the focal plane at $\approx 5 \mathrm{~m}$ downstream from the mirrors. Multiple step motors and a hexapod enabled the translation of the sample, the step size varied from typically $5 \mu \mathrm{m}$ to $10 \mu \mathrm{m}$. A $10 \times$ on-axis optical microscope was used for alignment and surveillance of the sample. The shape and size of the beam at the sample position were determined by a scintillator based x-ray-microscope. An evacuated tube of $7 \mathrm{~m}$ length spans the distance from the sample to the single photon counting pixel detector Pilatus 2M (50).

Fig. 10.1 $a$ shows a photograph of the Göttingen Instrument for Nano-Imaging with X-rays (GINIX) (23) at the P10-beamline of DESY's PETRAIII storage ring: A set of Kirkpatrick-Baez mirrors (not shown) enabled beam focusing to a size of about $200 \mathrm{~nm}$. The beam left the evacuated tube at (1) impinging the sample (here: a self-assembled glass-chamber, (4)) about $20 \mathrm{~cm}$ downstream from the mirrors. Two soft-edge apertures (3) block parasitic scattering. The sample was mounted on a motorized stage (5) enabling sample translation with respect to the beam. An on-axis video-microscope (OAV, Optique Peter, France) (7) with LED illumination (2) was used for orientation on the substrate. Optionally, cryogenic conditions (6) could be applied (Cryostream, Oxford instruments, UK). Diffraction patterns were recorded in $\approx 5.1 \mathrm{~m}$ distance by 
the single photon counting pixelated detector Pilatus 300k (Dectris, Switzerland). An evacuated tube (9) minimized absorption. The primary beam could be blocked by multiple semi-transparent beam stops placed either in front of the tube (8) or inside (not shown). The focused photon flux was $I_{0}=1.29 \cdot 10^{11} \mathrm{ph} . / \mathrm{s}$ as determined by direct beam recordings, see $b$. A representative cellular diffraction image is shown in $c$. Fig. 10.1 $d$ shows a photograph of the micro-SAXS data at cSAXS-beamline of the Swiss Light Source at the Paul Scherrer Institut in Villigen, Switzerland. The x-ray beam was focused by a $\mathrm{Si}(111)$ crystal monochromator in horizontal and a Rh-coated mirror in vertical direction enabling a spot size of about $40 \mu \mathrm{m}$ at the sample position. The beam left the vacuum tube through a $4 \mu \mathrm{m}$-thick mica-window at 10 impinging the sample (12) about $5 \mathrm{~m}$ downstream from the mirrors. Multiple step motors and a hexapod (11) enabled the translation of the sample with respect to the beam. An on-axis optical microscope 13 and a camera (16) were used for orientation on the substrate and the surveillance of the sample. The shape and size of the beam at the sample position was determined by a scintillator based x-ray microscope (15). An evacuated tube (14) of $7 \mathrm{~m}$ length spans the distance from the sample to the single photon counting pixelated detector Pilatus 2M [50). For x-ray measurements the on-axis microscope was retracted and the flight tube was approached to the specimen.

The focused photon flux was $I_{0}=1.44 \cdot 10^{11} \mathrm{ph}$./s as determined by direct beam recordings with filters, while the setup provides an extraordinarily clean direct beam signal, see $e$. A representative cellular diffraction image is shown in $f$. 
GINIX
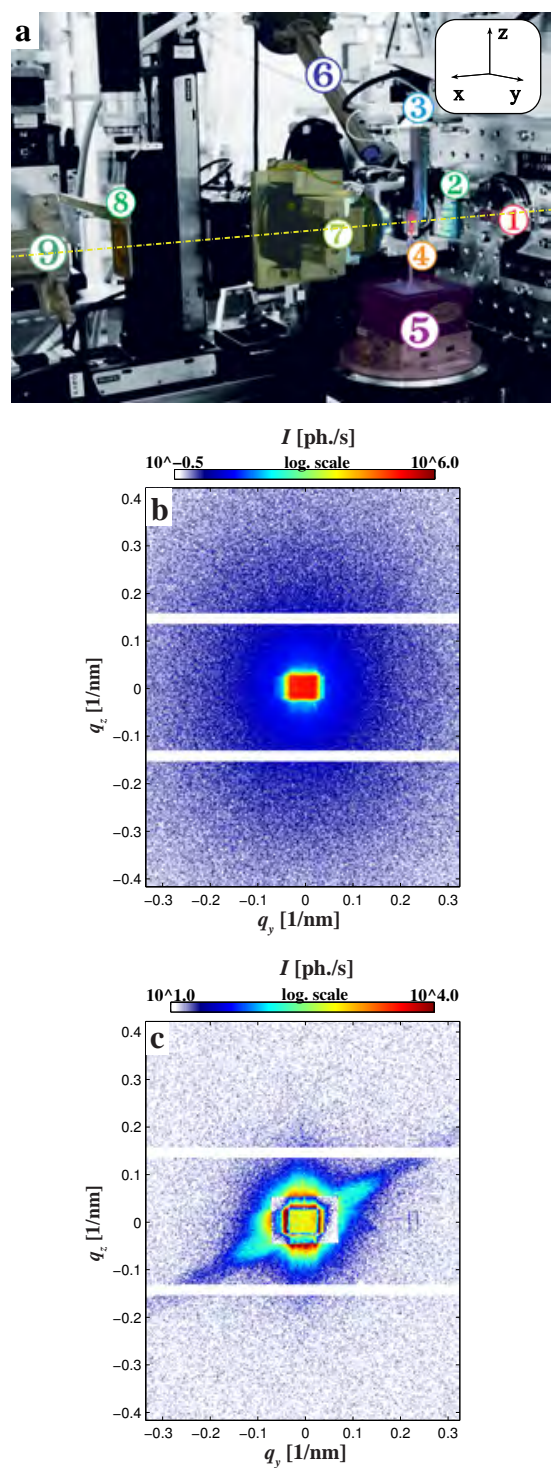

cSAXS
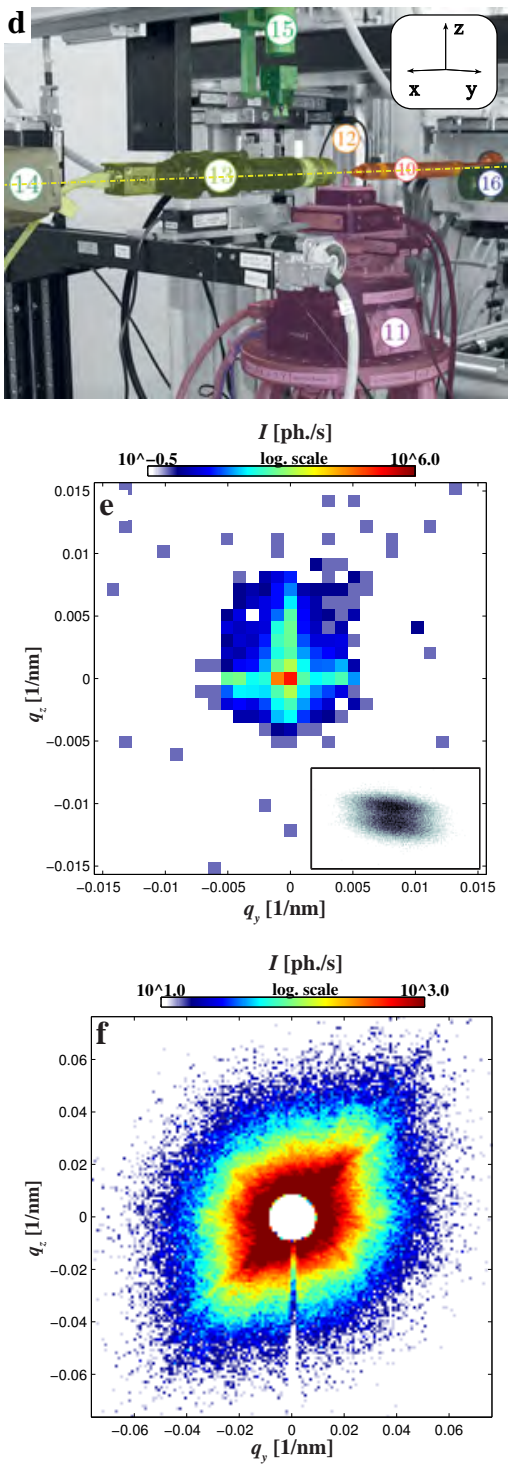

Figure 10.1: left: (a) Photograph of the nano-focus setup GINIX 20 23) together with far field images of (b) the direct beam and (c) a representative cellular diffraction signal recorded at GINIX. right: (d) Photograph of the micro-focus setup together with far field images of (e) the direct beam and (f) a representative cellular diffraction signal recorded at cSAXS 47). The beam profile in focus could be visualized by a scintillator, see e (inset). 


\subsection{PCA (Matlab Code)}

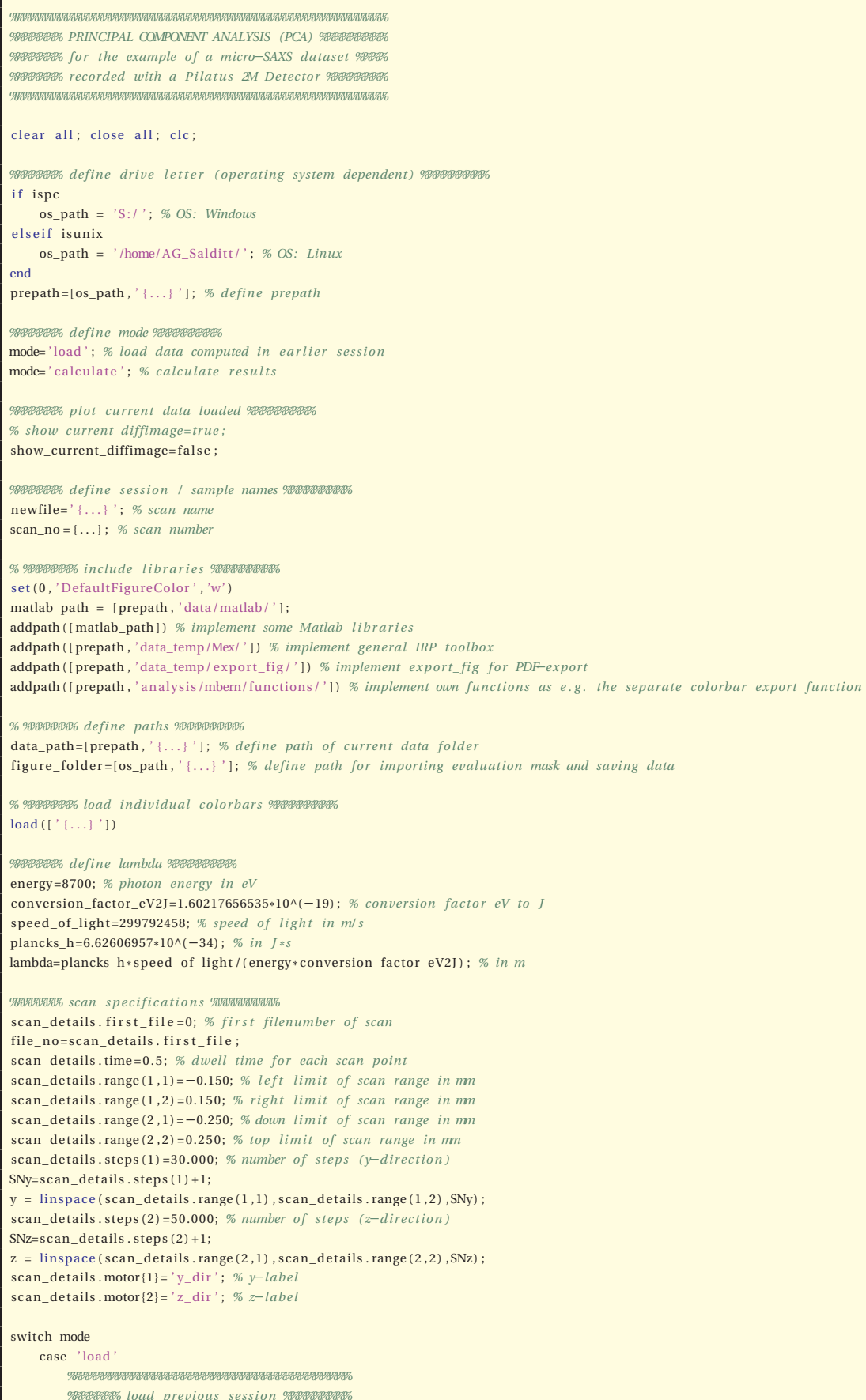




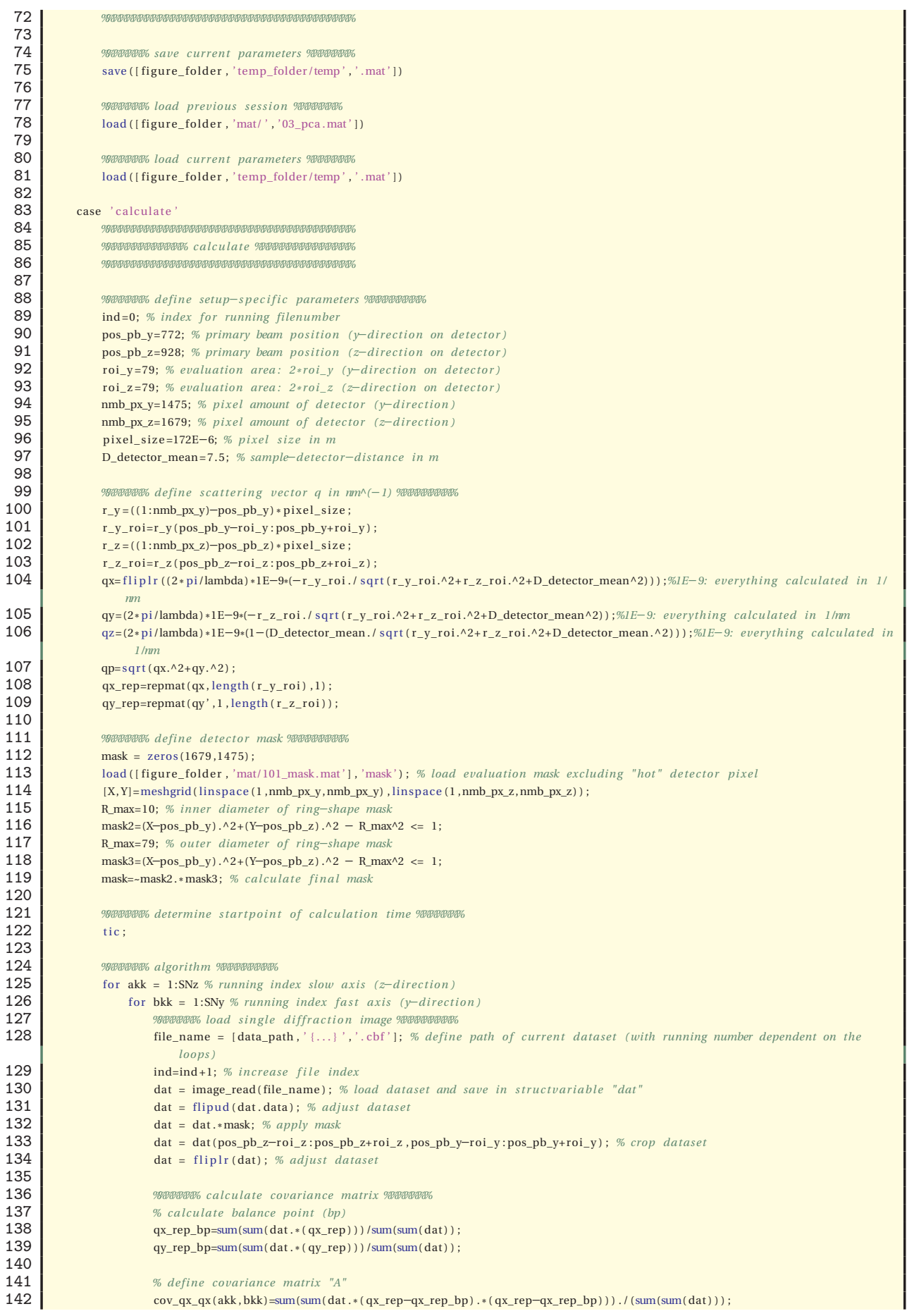


cov_qx_qy $($ akk , bkk) $=\operatorname{sum}(\operatorname{sum}($ dat $. *($ qx_rep-qx_rep_bp $) . *($ qy_rep-qy_rep_bp $))) . /($ sum $($ sum $($ dat $)))$; cov_qy_qx $($ akk, bkk) =cov_qx_qy $($ akk, bkk) :

cov_qy_qy (akk, bkk) =sum (sum (dat .* ( qy_rep-qy_rep_bp) .* ( qy_rep-qy_rep_bp ) ) )./ (sum (sum (dat) ) ) ; $\mathrm{A}=[$ cov_qx_qx (akk, bkk) cov_qx_qy (akk, bkk); cov_qy_qx (akk, bkk) cov_qy_qy (akk, bkk) ] ;

$\% \%$ solve eigenvalue problem: $V$, basetransform matrix; $D$, diagonal matrix with eigenvalues $\%$ $[\mathrm{V}, \mathrm{D}]=\operatorname{eig}(\mathrm{A})$

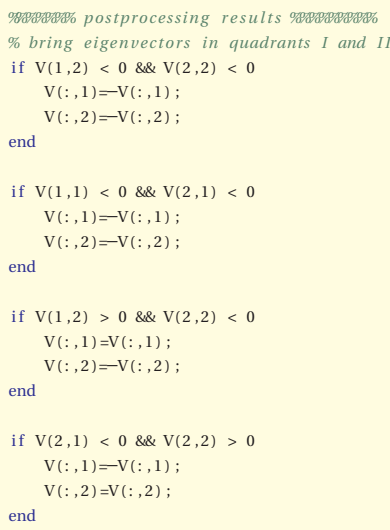




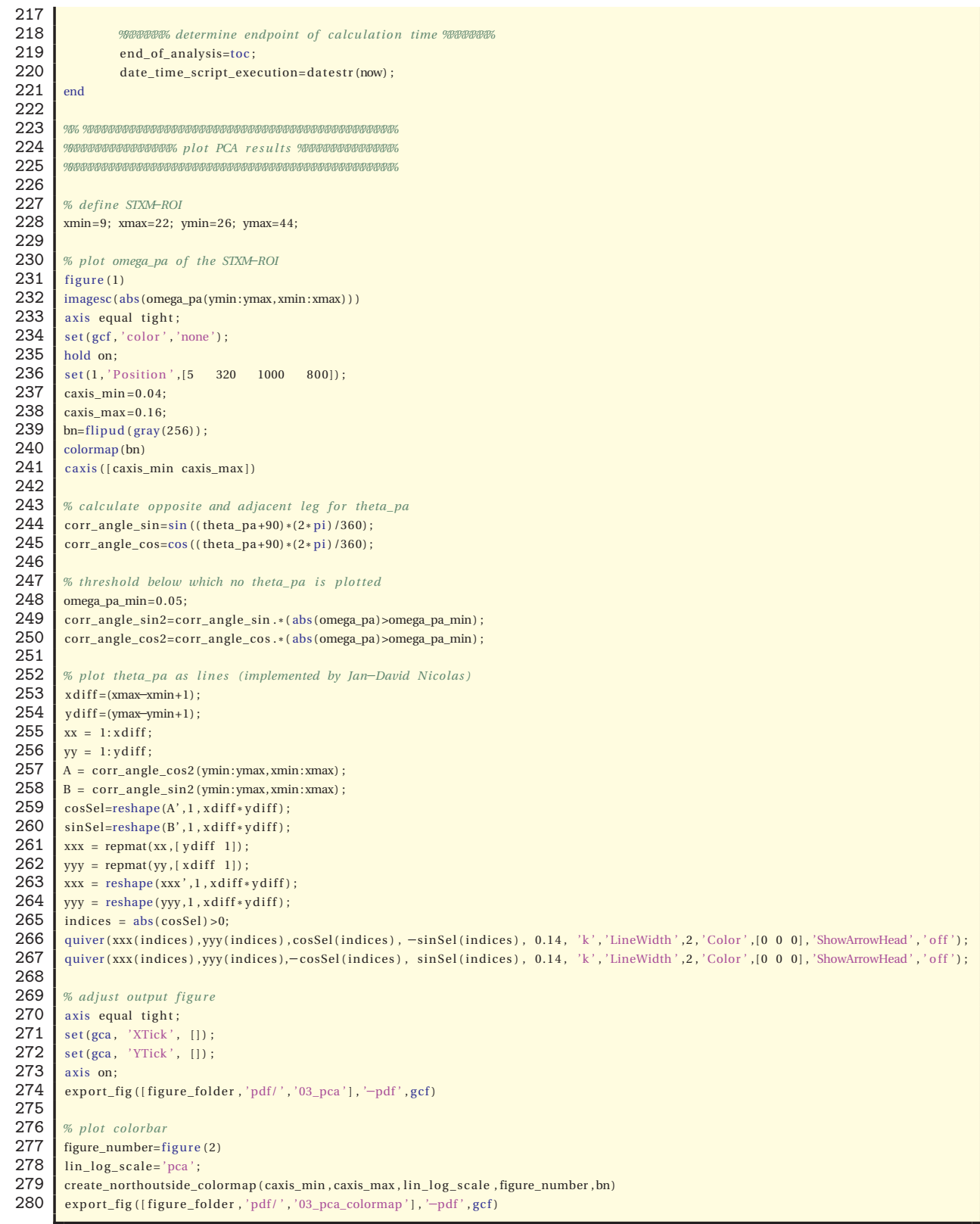




\subsection{Stem Cells: Statistics \& Cellular Diffraction Observ- ables}

This section is taken from (42).

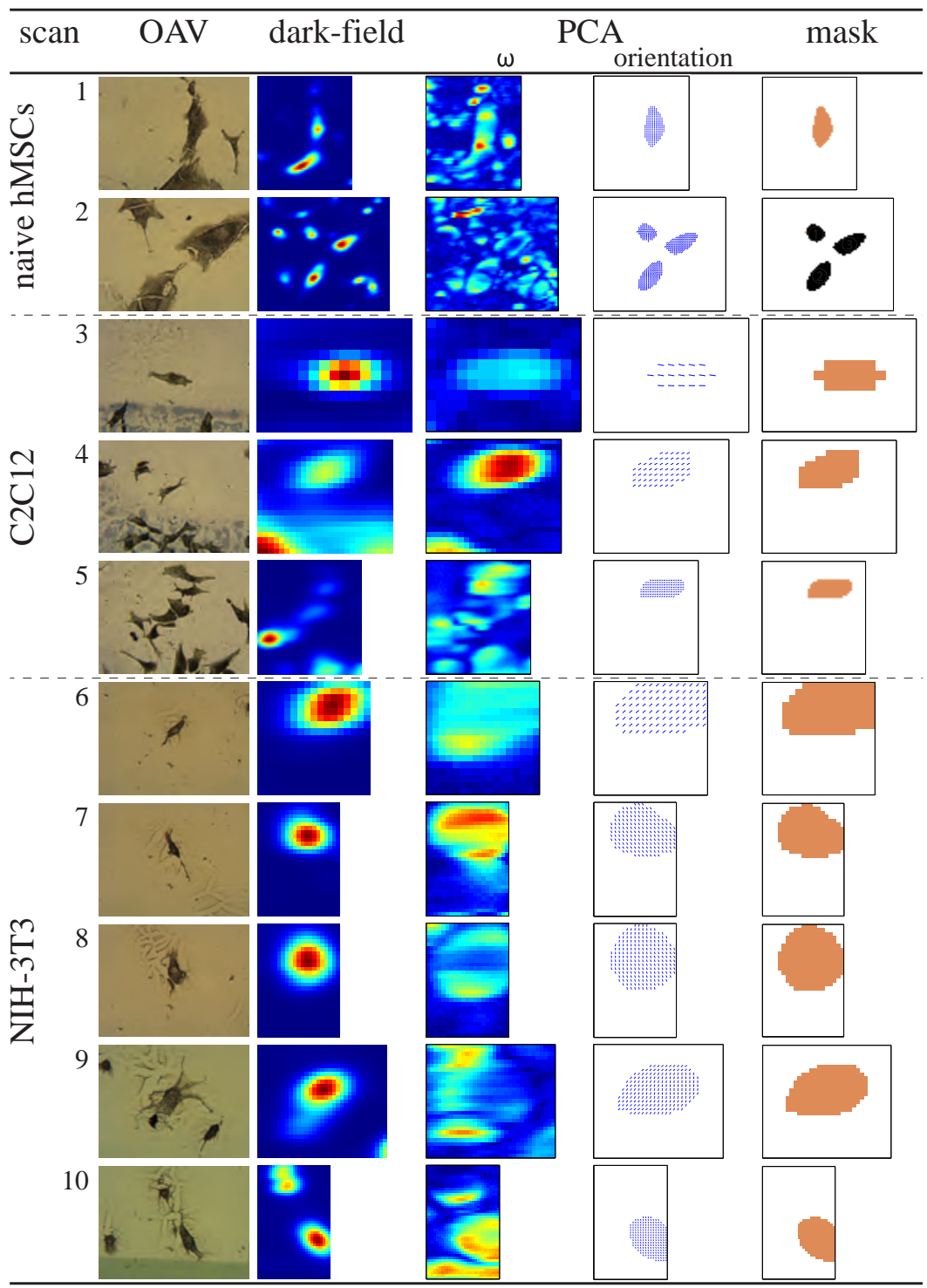




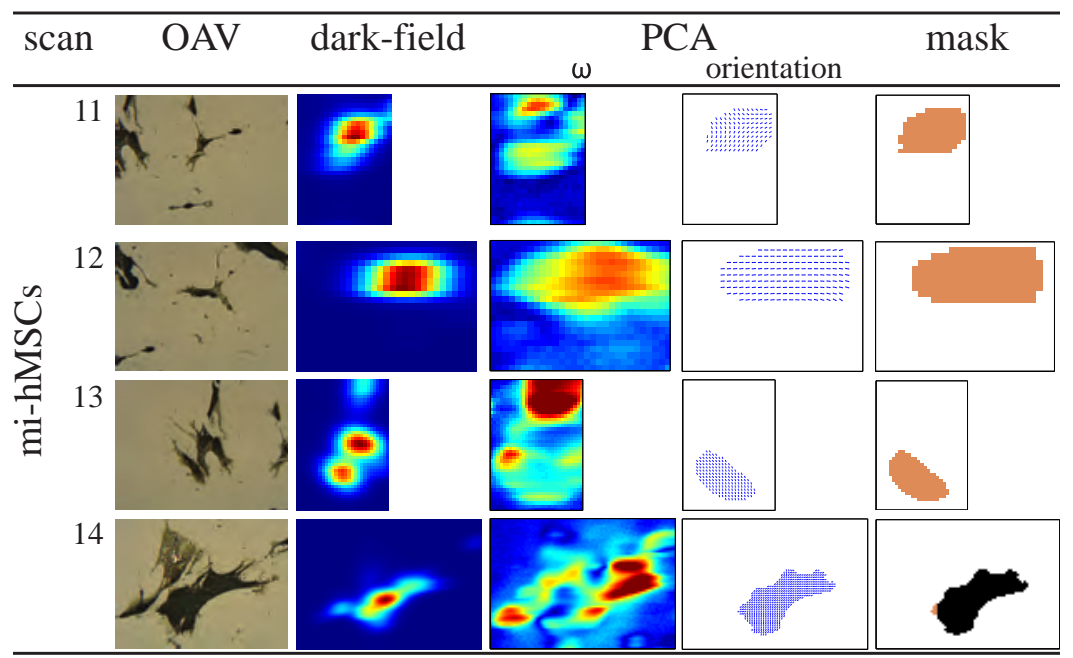

Figure 10.2: Overview of the datasets taken into account for statistical analysis showing the OAV-image in the $1^{\text {st }}$, the $x$-ray dark-field in the $2^{\text {nd }}$, the PCA order parameter and orientation in the $3^{\text {rd }}$ and the $4^{\text {th }}$ as well as the STXM masks for cells (red) in the $5^{\text {th }}$ column. Note, that for presentation reasons individual limits were set for color bars (not shown), so that a direct comparison between dark fields is not applicable. Figure and caption published in 42).

\begin{tabular}{|c|cc|ccc|ccccc|cccc|}
\hline cell type & \multicolumn{3}{|c}{ naive hMSC } & \multicolumn{4}{|c}{ C2C12 } & \multicolumn{4}{c|}{ NIH-3T3 } & \multicolumn{4}{c|}{ mi-hMSC } \\
scan no & $\mathbf{1}$ & $\mathbf{2}$ & $\mathbf{3}$ & $\mathbf{4}$ & $\mathbf{5}$ & $\mathbf{6}$ & $\mathbf{7}$ & $\mathbf{8}$ & $\mathbf{9}$ & $\mathbf{1 0}$ & $\mathbf{1 1}$ & $\mathbf{1 2}$ & $\mathbf{1 3}$ & $\mathbf{1 4}$ \\
\hline$\Delta_{y}$ in $\mu \mathrm{m}$ & 8 & 10 & 10 & 5 & 5 & 5 & 5 & 5 & 5 & 5 & 5 & 5 & 5 & 5 \\
$\Delta_{z}$ in $\mu \mathrm{m}$ & 8 & 10 & 10 & 5 & 5 & 5 & 5 & 5 & 5 & 5 & 5 & 5 & 5 & 5 \\
\hline
\end{tabular}

Table 10.1: Scan step sizes for data shown in Fig. 10.2 and further analyzed in Sec. 4.2 All cells are grown on $1 \mu \mathrm{m}$-thick $\mathrm{Si}_{3} N_{4}$-membranes, cryoprepared at alive state and then freeze-dried. All scans are performed at an energy of $E_{\mathrm{ph} .}=8.7 \mathrm{keV}$ with a dwell time of $t_{\mathrm{dwell}}=0.5 \mathrm{~s}$ and a flux of $I_{0}=$ $1.44 \cdot 10^{11} \mathrm{ph} . / \mathrm{s}$ using the instrumentation of the cSAXS endstation. Figure and caption adapted from 42 . 


\subsection{Grid Plunger Protocol}

An adequate plunging procedure is essential for any sample preparation involving a vitrification step which fixates single cells or thin tissue slices (even without the use of any chemical agent) in a morphologically meaningful state by rapid (shock-) freezing of the specimen. In a subsequent freeze-drying step one can remove all volatile components of the biomaterial and thus enhance the x-ray contrast of the remaining biological matter, such as the cytoskeletal network of cells.

However, the most critical step of the preparation procedure is the time between blotting the sample and plunging: On the one hand, if the blotting on the cell-containing side takes too long or is fully completed, the sample might well dry out. On the other hand, if the remaining solution layer is too thick, the cooling rate of the plunging bath will not be sufficient so that the sample will be covered with ice crystals. In order to avoid both scenarios, a reliably functioning climate chamber (especially with respect to the humidity) is of the essence. We have learned that experience on one particular instrument such as the Leica Grid Plunger EM GP with all its features, bugs, specifications as well as its individual handling is decisive to reliably well-prepare samples.

The following introduction is the result of a long-lasting successive learning process and will describe, among other aspects, how to preserve a sample during the plunging process with a Leica Grid Plunger as well as how to trouble-shoot, if the chamber fails to hold the humidity value that is predefined in the menu:

There are three parameters that can be set for the plunging session on the Leica GP panel, which are:

- The temperature of the climate chamber $\mathrm{T}_{C}$

- The humidity of the climate chamber $\mathrm{H}_{R}$

- The temperature of the plunging bath

Typically, setting the $\mathrm{T}_{C}$ value to $20^{\circ} \mathrm{C}$ (about room temperature) for chemically fixated samples (stored in the refrigerator) and alive samples (from the incubator at $37^{\circ} \mathrm{C}$ ) is a good choice, because thus fogging of the glass window from the inside (in case of setting $\mathrm{T}_{C}$ to $37^{\circ} \mathrm{C}$ ) can be avoided and the temperature can be held approximately constant throughout the entire plunging process. Moreover, $20^{\circ} \mathrm{C}$ is well-tolerable for alive cells to a certain extent.

In order to avoid any out-drying of the specimen, the highest humidity that can be applied to the chamber is beneficial for the plunging procedure. However, when setting $\mathrm{H}_{R}$ to $99 \%$, we have experienced problems: Randomly during the process the humidity regulation may fail in an instant. According to the manufacturer, the humidifier merely provides an on- and an off-state. When the humidifier is turned on, and if the measured humidity is correct at all times, still remains elusive for us. However, using the 
experience we gathered, we have presumed the humidity to be sufficiently high, if the humidifier is constantly producing thick white steam throughout the entire time of the running of the instrument (with the exception of a short acclimatization time right at the beginning). In this regard, we have applied (i) a change in the menu, (ii) a change in our preparation workflow as well as (iii) established a procedure in order to reliably recover the humidity in case of a failure of the humidifier.
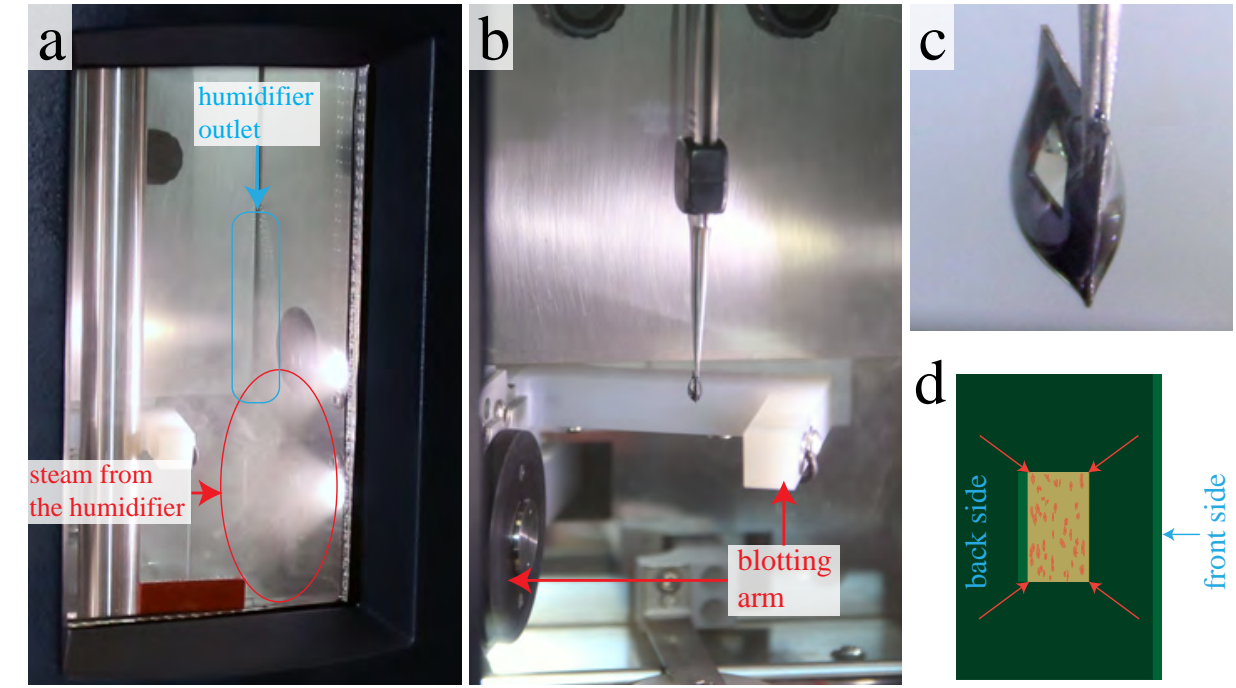

Figure 10.3: (a) Photograph of the climate chamber showing thick white steam indicating, that the humidifier works properly. (b-c) Sample mounted in the chamber covered by a droplet in order to prevent any uncontrolled drying of the adhered cells. (d) Sketch of a SiRN-window (back side view) showing the Si-framework in green and the SiRN-membrane in yellow. Red arrows indicate the points of support of the blotting stick when blotting from the back side.

(i-ii) Change in menu \& in preparation workflow: There are in total five positions the plunger is successively put through during a plunging cycle: 1 . load forceps, 2 . lower chamber, 3. blot, 4. plunge and 5. transfer. The blotting step of position 3. is typically used for automated blotting using the blotting arm of the instrument. This step is useless for our "unconventional" preparation of SiRN-windows, which requires manual blotting with filter paper on a tweezer through one of the side ports. However, the step cannot be eliminated in the menu and shall thus be applied (without any effect) before the actual (manual) blotting step in order to avoid an additional time delay between blotting and plunging.

Moreover, the pre-defined settings in the menu instruct the humidifier to be switched off after the automatic blotting step in order to provide the user a clear view on the sample. This is counterproductive for our individual preparation procedure, because it shuts down the production of additional humidity when the sample is shortly before 
drying out. In order to change this setting, follow these steps on the panel:

setup $\rightarrow$ settings $\rightarrow$ service $\rightarrow$ code: $1170 \rightarrow$ control settings

$\rightarrow$ uncheck "Fan off before plunge"

$\rightarrow$ uncheck "Humidifier off before plunge"

When mounting the sample, we now cover the membrane entirely in a droplet of buffer solution, which ensures that the cells stay safely wet right until the blotting step. For blotting, the (cell-free) back side of the substrate has to be identified by pressing the "manual rotation"-button and looking through the microscope; the back side has to be blotted first. In case of the cells being placed on the flat side of a SiRN-window, the back side is thus the deepened side of the window: Go into all four corners of the inner Si-framework. Pay attention to the capillary effects of the tweezer, which occasionally transfers buffer solution from the front- to the back side. This requires additional blotting steps of the back side. In this process, the front side has now to be constantly inspected in view of a buffer layer still being present. After drying the back side, the front side can now be blotted and the sample can be plunged in an instant. Make sure, that before blotting the front side, the automatic blotting step is already carried out. In the ideal case, the critical time between applying the blotting stick for the last time and plunging can thus be minimized to $1-3$ sec.

Fig. 10.3 shows selected photographs and a sketch illustrating some of the details of the plunging procedure as described above: Fig. 10.3 (a) shows the climate chamber with the working humidifier as can be recognized by the production of thick white steam coming from the rear right of the chamber. $(b-c)$ Photograph of a mounted SiRNwindow covered by a droplet in order to keep the sample safely wet until shortly before plunging. In the case of using a SiRN-window, cells are let to settle on the flat side and major parts of the droplet are first removed by blotting from the back side going into all four corners of the inner Si-framework (red arrows), see $(d)$. Remaining parts from the front side are then removed just before plunging.

(iii) Recovering the humidity: If the climate chamber fails to produce steam before or within this process, recover by carrying out the following steps:

1. Play with the water level of the humidifier bath using a syringe. There is an ideal water level range of the humidifier bath, where the ultrasonic evaporator starts to work again.

2. If this does not solve the problem, save your sample by reapplying a droplet of buffer solution and go back to the loading position. Play again with the water level.

3. If this still does not solve the problem, disassemble the humidifier bath and check, if the two connectors are still dry.

Although the protocol sounds a little unconventional, when carrying out these steps 
we were always able to get the humidifier back working again. All further details of the instrument can be taken from the instruction manual of the Leica Grid Plunger (71) or are self-explanatory.

\section{Finally, some remarks about the sample handling \& the plunging bath:}

cryovial design: Fig. $10.4(a-b)$ show a photograph of the adapted cryovials we have used for sample transfer and storage of SiRN-windows in liquid nitrogen. The design was conceived by my former colleague Dr. Marius Priebe and is as follows: A standard cryovial is opened and its cap is perforated using a drill in order to avoid the accumulation of gas bubbles. Together with two metal nuts, which are placed inside the vial, this design ensures, that the vial can be submersed and stays under the liquid nitrogen level. Furthermore, a truncated syringe tip positions the substrate upright and fixes it, so that gas bubbles cannot break the sample window or be trapped on the cellcontaining front side of the substrate, in particular during the freeze-drying process.
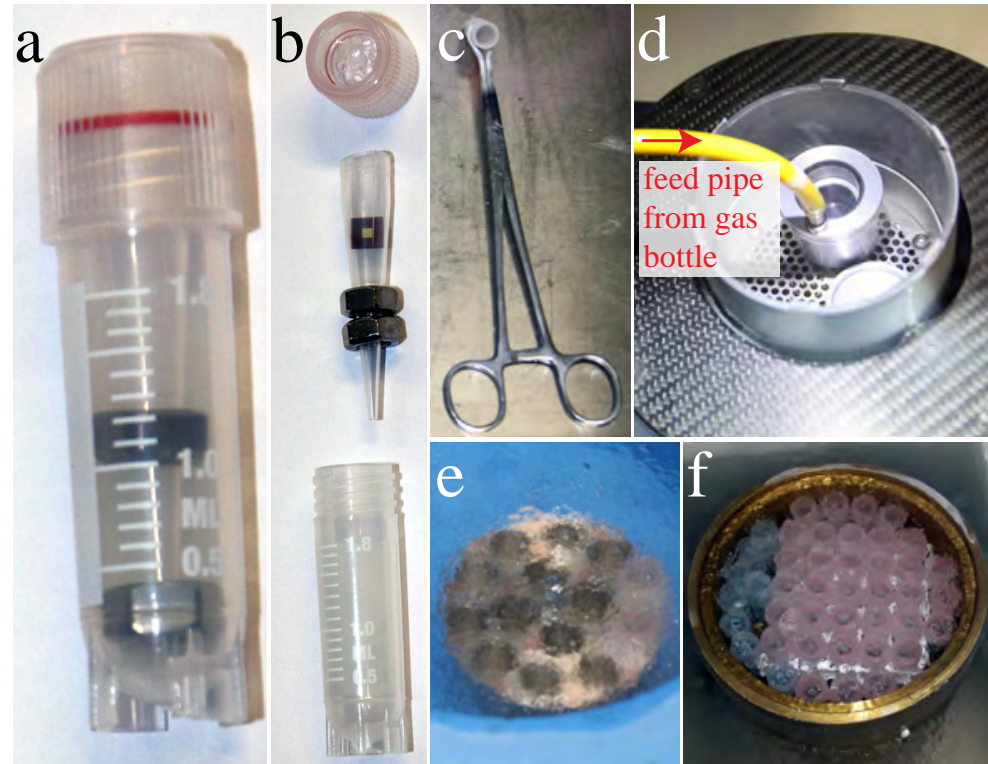

Figure 10.4: $(a-b)$ Photographs of the modified cryovials used for samples prepared on small SiRN-windows (outer edge length: $5 \mathrm{~mm}$ ). Further details see text. (c) Photograph of one of the three forceps (with different grabbing angles) which were used in order to transfer the cryovials into liquid nitrogen. (d) Photograph of the condensation cap used to fill the plunging bath. (e-f) Cryovials submersed in liquid nitrogen were stored in a metal revolver short after plunging, see (e) and were transferred into a plastic box mounted in a metal cylinder shortly before freeze-drying, see ( $f$ ).

Cryomarker: In order to relocate a particular region on a sample, e.g. as required for the comparative analysis approach described in Sec. 3.2 it is beneficial to mark a particular corner of the front side of the substrate. However, when using tweezers to grab the substrate, one may apply mechanical forces to the marked area with the 
effect of potentially disrupting marker parts and spill them over the SiRN-window. In order to be able to distinguish cryomarker from cellular structures on the freeze-dried substrate, one should use a (red-) colored marker (as e. g. a Secureline fineline marker II superfrost).

Filling the plunging bath: A mixture of ethane/propane can achieve temperatures around $-195^{\circ} \mathrm{C}$ without any freezing, while pure ethane only allows temperatures of around $-185^{\circ} \mathrm{C}$. Using the ethane/propane-mixture gives the experimenter an additional 'range' to prepare the instrument during the filling procedure and is thus advantageous. When re-filling the plunging bath due to a long-lasting preparation, one has to make sure, that the corresponding metal adapter (as shown in Fig. $10.4(d)$ ) is dry. Any remaining moisture inside of the pipe and adapter has to be removed by blow-drying, because after cooling it down, an ice crystal formation will block the feed pipe otherwise.

Maintenance of the climate chamber: In between a longer preparation period, the humidity of the chamber will condense on the chamber's walls to a significant extent. From time to time it is therefore necessary to dry the bottom of the chamber with wipes in order to avoid water drops falling into the plunging bath when applying the plunging step.

Quality check as regards ice crystal formation: A good quality check of the plunging procedure is to review the sample during transfer out of the plunging bath: If the cover layer of the sample window is still transparent, one can assume that the plunging works sufficiently well. However, if it is (locally) covered with a white layer one can assume the formation of ice crystals. Refilling of the cryovial to the rim (by e.g. using another cryovial and shipping over additional liquid nitrogen), before performing the transfer (with the open vial) to the Dewar vessel will give additional safety to preserving the sample.

Sample storage: Samples should be stored in metal frameworks as e.g. shown in Fig. 10.4 (e), because the heat capacity of the metal (together with the heat capacity of the two nuts inside the vials) provides extra time for the transfer into a Dewar vessel, a dry shipper or the metal cylinder used for the freeze-drying process (see Fig. 10.4 (e)). The upright position of the cryovials as provided by a plastic box inside the cavity of a (partially hollow) metal cylinder ensures, that liquid nitrogen gas can evaporate during the process and does not remain in an uncontrolled position within the vial, which might damage the sample itself. 


\subsection{Mobile Incubator}

Alive recordings at synchrotron beam lines typically require a transport of cells to the facility. Once reaching the campus, the samples can be transferred back into a stationary incubator. Nowadays, most synchrotrons provide biological S1 labs including an incubator as well as a clean bench. In order to realize the transport, our electronics department "Elektronikwerkstatt des Instituts für Materialphysik" has kindly conceived and constructed a mobile incubator, which can hold temperatures between about $5{ }^{\circ} \mathrm{C}$ (for refrigerated samples) up to $37^{\circ} \mathrm{C}$ and above (for alive samples), and provides plugs for the $12 \mathrm{~V}$ cigarette lighters of a car as well as the standard $230 \mathrm{~V}$ circuits of a train.
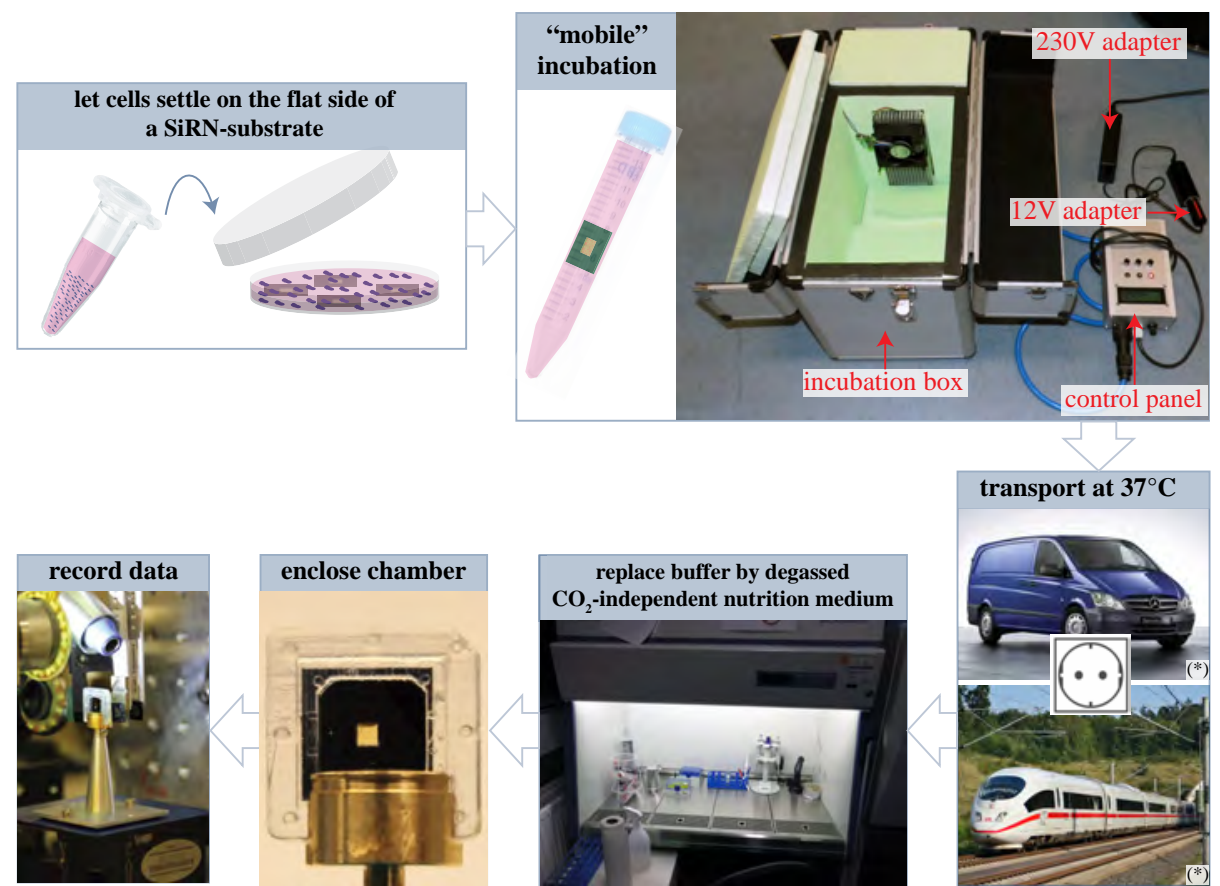

Figure 10.5: Workflow for an alive cell transport from the institute to a synchrotron beam line. Photographs marked with $\left(^{*}\right)$ are taken from source [72] \& 73].

Fig. 10.5 shows the transfer and mounting procedure as applied on the alive cell samples of Sec. 5.4. Cells are let to settle on SiRN-substrates in our stationary incubator. Shortly before the shipment to the synchrotron, the cells are transferred into a vial covered with medium and placed in the mobile incubator. The samples are delivered to the synchrotron right in time. We have then replaced the medium by degassed $\mathrm{CO}_{2}$ independent medium in order to avoid gas bubble formation during the measurement. 
Note, that $\mathrm{CO}_{2}$-dependent medium is not an option at this point, because degassing the buffer would shift the steady state equilibrium of the bicarbonate buffer system towards the production of more $\mathrm{CO}_{2}$ and would thus shift the $\mathrm{pH}$ towards more alkaline values:

$$
2 \mathrm{H}^{+}+\mathrm{CO}_{3}^{2-} \rightleftharpoons \mathrm{H}^{+}+\mathrm{HCO}_{3}^{-} \rightleftharpoons \mathrm{H}_{2} \mathrm{CO}_{3} \rightleftharpoons \mathrm{CO}_{2} \uparrow+\mathrm{H}_{2} \mathrm{O}
$$

We have then closed the SiRN-chamber and inspected the sample window under a microscope. The sealed chamber is then washed from the outside with $\mathrm{H}_{2} \mathrm{O}$, which removes observed dirt spots on the sample window and then directly mounted on the sample stage of the beam line in order to start the recording. 